\begin{abstract}
RINKU MUKHERJEE. Post-Stall Prediction of Multiple-Lifting-Surface Configurations Using a Decambering Approach. (Under the direction of Dr. Ashok Gopalarathnam.)

A novel scheme is presented for an iterative decambering approach to predict the post-stall characteristics of wings using known section data as inputs. The new scheme differs from earlier ones in the details of how the residual in the Newton iteration is computed. With earlier schemes, multiple solutions are obtained for wings at high angles of attack as the final converged solution depends on the initial conditions used for the iteration. With this scheme, multiple solutions at high angles of attack are brought to light right during the computation of the residuals for the Newton iteration. In general, the new scheme is found to be more robust at achieving convergence. Experimental validation is provided using experimental airfoil lift curves from Naik and Ostowari for three different aspect ratios of rectangular wings. Results are presented from a study of the stall characteristics of wings of different planform shapes and two configurations of a wing-tail and a wing-canard configuration. Results are also presented from a study to investigate possible asymmetric lift distributions when the iterations were started with an initial asymmetric distribution of the decambering.
\end{abstract}




\title{
Post-Stall Prediction of Multiple-Lifting-Surface Configurations Using a Decambering Approach
}

\author{
by \\ Rinku Mukherjee \\ A dissertation submitted to the Graduate Faculty of \\ North Carolina State University \\ in partial fulfillment of the \\ requirements for the Degree of \\ Doctor of Philosophy
}

Aerospace Engineering

Raleigh, NC

2004

APPROVED BY:

Dr. Ashok Gopalarathnam

Advisory Committee Chairman

Dr. Jack R. Edwards

Advisory Committee Member
Dr. Fen Wu

Advisory Committee Member

Dr. Zhilin Li

Advisory Committee Member 
To Sudipto...... 


\section{BIOGRAPHY}

Rinku Mukherjee had her primary education in India. She graduated with a Bachelor's in Civil Engineering from Jadavpur University, Kolkata, India in 1998. She got her Master's in Ocean Engineering and Naval Architecture from the Indian Institute of Technology Kharagpur, India in 2001. She joined North Carolina State University in 2001 to pursue a PhD in Aerospace Engineering. 


\section{ACKNOWLEDGEMENTS}

I extend my deepest gratitude to my advisor Dr. Ashok Gopalarathnam for his untiring support and encouragement without which this thesis would not be complete. He has taken personal efforts to provide guidance and both emotional and academic support during my time under him as a graduate student. Whether it was studying courses, writing conference papers, preparing for presentations, writing resumes or plain handling oneself professionally, he has always extended his undivided attention to every minute detail and helped me to become better than myself. His constant perseverance to do things better has been a great source of learning. In spite of his very busy schedules he found the time to give personal attention to every student in his continually growing research group. The greatest lesson that I have learnt from him as a researcher is to say "I do not know". To acknowledge that one "does not know" and not fear the unknown can only lead to further and rigorous research and is perhaps one of the greatest qualities to possess as a researcher. Also, Dr. Gopalarathnam continually refers to research as "fun" and $C_{l^{-}} \alpha$ plots as "beautiful" !!! That added so much colour to life and made research lot more fun.

I am grateful to Dr. Jack R. Edwards, Dr. Fen Wu and Dr. Zhilin Li for being on my doctoral committee.

I thank Dr. Jack Edwards for his suggestions and invaluable advice in dealing with some of the very tricky problems in this research.

This research effort was supported under Grant NAG-1-01119 from the NASA Langley Research Center. This support and helpful discussions with the technical monitor, SungWan Kim, are gratefully acknowledged.

I thank my colleagues in my research group for making my graduate days less stressful.

Thanks are overly due to my mother for her unconditional love and unrelenting 
support for everything that I have pursued in my life. Thanks are due to my kid sister without whose support, love and taking on more than her share of the responsibilities, my academic pursuits would be incomplete. Thanks are due to my late father who taught me to reach for the skies. My heartfelt thanks are due to my parents-in-law for their unflinching love, care and support. Finally, I would not be even writing this thesis without the love and care of my lifelong friend, my husband, Sudipto and I could never thank him enough for always being there for me. 


\section{Table of Contents}

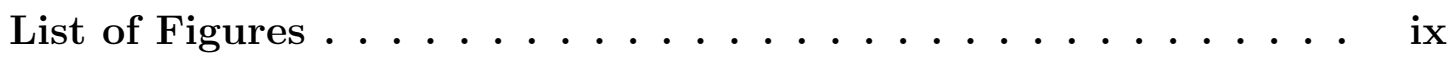

Nomenclature .................. xiii

Chapter 1 Introduction $\ldots \ldots \ldots \ldots \ldots \ldots \ldots$

1.1 Literature Study . . . . . . . . . . . . . . . . . . . 2

1.1.1 The Iterative $\Gamma$ distribution Approach . . . . . . . . 3

1.1.2 The $\alpha$ Correction Approach . . . . . . . . . . 7

1.2 Current Approach . . . . . . . . . . . . . . . . . . . 9

Chapter 2 Illustration of the Decambering Concept for Flow Past an Airfoil ..................... 10

2.1 The Decambering Concept . . . . . . . . . . . . . . . . . . 11

Chapter 3 Post-stall prediction of a finite wing $\ldots \ldots \ldots 20$

3.1 Decambering for a wing . . . . . . . . . . . . 20

3.1 .1 Vortex Lattice Method(VLM) . . . . . . . . . . . 21

3.1.2 Predicting the decambering along wing span . . . . . . 21

3.2 The iteration procedure $\ldots \ldots \ldots \ldots \ldots$

3.3 Multiple intersections in scheme $2 \ldots \ldots \ldots \ldots$

Chapter 4 Results ................... 31

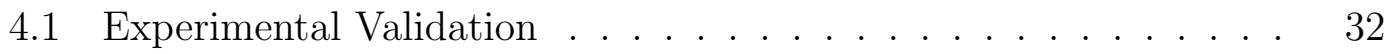


4.1.1 Rectangular Wing $(\mathrm{AR}=12)$ with the NACA 4415 Airfoil at Re of 0.5 Million .............. . . 33

4.1.2 Effect of initial conditions on the iterations for Scheme 2 . 40

4.1.3 Rectangular Wing $(\mathrm{AR}=9)$ with the NACA 4415 Airfoil at Re of 0.5 Million ............... . . 41

4.1.4 Rectangular Wing $(\mathrm{AR}=6)$ with the NACA 4415 Airfoil at Re of 0.5 Million . . . . . . . . . . . . 43

4.1.5 Rectangular Wing $(\mathrm{AR}=12)$ with the NACA 4415 Airfoil at Re of 0.75 Million . . . . . . . . . . . . 45

4.1.6 Rectangular Wing $(\mathrm{AR}=9)$ with the NACA 4415 Airfoil at Re of 0.75 Million . . . . . . . . . . . . 47

4.1.7 Rectangular Wing $(\mathrm{AR}=6)$ with the NACA 4415 Airfoil at Re of 0.75 Million . . . . . . . . . . . . . . 49

4.1.8 Changes to the Lift Curve with Change to Aspect Ratio . 51

4.1 .9 Summary . . . . . . . . . . . . . . 56

4.2 Study of stall characteristics . . . . . . . . . . . . . . . 57

4.2.1 Stall Characteristics of a Part-Tapered Wing . . . . . . . 63

4.3 Wing-Tail Configuration ... . . . . . . . . . . . 65

4.4 Wing-Canard Configuration . . . . . . . . . . . . . . 70

4.5 Spanwise Asymmetry in the Initial Conditions . . . . . . . . . . . 74

Chapter 5 Conclusions ................. 82

Chapter 6 Future Work ................. 86

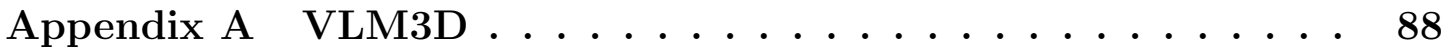

A.0.1 Subroutine readgeom . . . . . . . . . . . . . 88

A.0.2 Subroutine geomgen ................ 89 
A.0.3 Subroutine influence . . . . . . . . . . . . . . . . . . 90

A.0.4 Subroutine readoper. . . . . . . . . . . . . . . 92

A.0.5 Subroutine dorhs . . . . . . . . . . . . . . . . . . . . . . 92

A.0.6 Subroutine iteration . . . . . . . . . . . . . . . . . . . . 92

References ..................... 95 


\section{List of Figures}

2.1 Flow separation from an airfoil at a high angle of attack. . . . . .

2.2 Schematic diagram of functions 1 and $2\left(\delta_{1}\right.$ and $\delta_{2}$ are negative as shown) used to model effective decambering of an airfoil. . . . . . 12

$2.3 C_{l}$ and $C_{m}$ of the NACA 0012 airfoil. . . . . . . . . . . . . . 13

$2.4 C_{l}$ and $C_{m}$ using potential-flow with decambering. . . . . . . . . 14

2.5 The $\alpha$ s chosen to illustrate effectiveness of decambering. . . . . . 15

2.6 Effectiveness of the decambering for $\alpha$ of $10 \mathrm{deg}$. . . . . . . . . 16

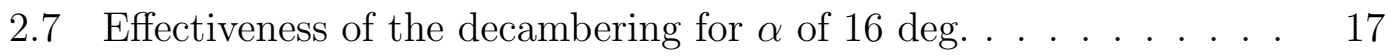

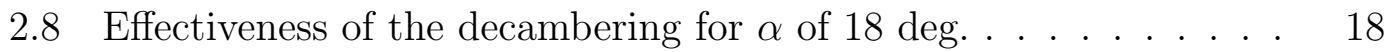

2.9 Flow chart of the iterative decambering approach in 2D flow. . . . 19

3.1 Vortex Lattice Method(only six lattices shown for clarity). . . . . 22

3.2 Flow chart of the iterative decambering approach for a wing(s). . $\quad 28$

3.3 Illustration of the differences in the computation of the residuals using schemes 1 and 2. . . . . . . . . . . . . . . 29

3.4 Illustration of the different ways in which a trajectory line may intersect the airfoil $C_{l}$ - $\alpha$ curve. . . . . . . . . . . . . . . . 29

3.5 Flow chart for handling multiple intersections in Scheme 2 at a particular section on the wing(s). . . . . . . . . . 30

4.1 Airfoil lift curves for the NACA 4415 airfoil from Naik and Ostowari ${ }^{17}$. 33

4.2 Planform of the rectangular wings (RHS shown) used in sec. 4.1. . 34

4.3 Wing $C_{L^{-}} \alpha$ predicted using schemes 1 and 2 for a rectangular wing of aspect ratio 12, using a NACA 4415 airfoil at Reynolds number of 0.5 million. . . . . . . . . . . . . . . . . . . .

4.4 Spanwise $C_{l}$ distribution predicted for a rectangular wing of aspect ratio 12, using a NACA 4415 airfoil at Reynolds number of 0.5 million from scheme 1. . . . . . . . . . . . . . . .

4.5 Spanwise $C_{l}$ distribution predicted for a rectangular wing of aspect ratio 12, using a NACA 4415 airfoil at Reynolds number of 0.5 million from scheme 2. . . . . . . . . . . . . . .

4.6 Wing $C_{L}$ variation with number of iterations for a rectangular wing of aspect ratio 12, using a NACA 4415 airfoil at Reynolds number of 0.5 million from scheme $2 \ldots \ldots \ldots \ldots$. . . . . . . . . . . . . . . . . 
4.7 Sawtooth in spanwise $C_{l}$ distribution for a rectangular wing of aspect ratio 12, using a NACA 4415 airfoil at Reynolds number of

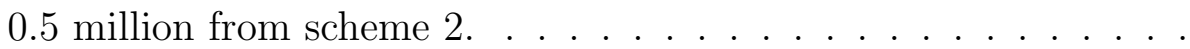

4.8 Location of the upper and lower corners of the sawtooth region shown in Fig. 4.7 on the NACA 4415 airfoil $C_{l^{-}} \alpha$ curve. . . . . . .

4.9 Wing $C_{L^{-}} \alpha$ predicted for a rectangular wing of aspect ratio $12 \mathrm{using}$ a NACA 4415 airfoil at Reynolds number of 0.5 million for different starting values of $\delta_{1} \ldots \ldots \ldots \ldots \ldots$

4.10 Spanwise section $C_{l}$ predicted for a rectangular wing of aspect ratio 12 using a NACA 4415 airfoil at Reynolds number of 0.5 million for different starting values of $\delta_{1} \ldots \ldots$. . . . . . . . . .

4.11 Wing $C_{L^{-}} \alpha$ predicted using schemes 1 and 2 for a rectangular wing of aspect ratio 9, using a NACA 4415 airfoil at Reynolds number of 0.5 million.

4.12 Spanwise $C_{l}$ distribution predicted for a rectangular wing of aspect ratio 9, using a NACA 4415 airfoil at Reynolds number of 0.5

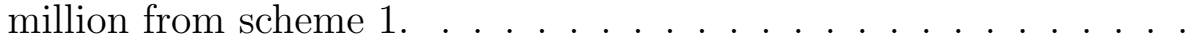

4.13 Spanwise $C_{l}$ distribution predicted for a rectangular wing of aspect ratio 9, using a NACA 4415 airfoil at Reynolds number of 0.5 million from scheme 2. . . . . . . . . . . . . . . .

4.14 Wing $C_{L^{-}} \alpha$ predicted using scheme 2 for a rectangular wing of aspect ratio 6, using a NACA 4415 airfoil at Reynolds number of 0.5 million. . . . . . . . . . . . . . . . . . . . . . .

4.15 Spanwise $C_{l}$ distribution predicted for a rectangular wing of aspect ratio 6, using a NACA 4415 airfoil at Reynolds number of 0.5 million from scheme 2 .

4.16 Wing $C_{L^{-}} \alpha$ predicted using schemes 1 and 2 for a rectangular wing of aspect ratio 12, using a NACA 4415 airfoil at Reynolds number of 0.75 million.

4.17 Spanwise $C_{l}$ distribution predicted for a rectangular wing of aspect ratio 12, using a NACA 4415 airfoil at Reynolds number of 0.75

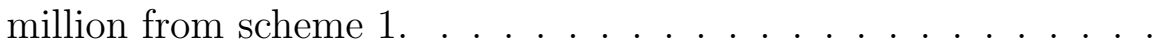

4.18 Spanwise $C_{l}$ distribution predicted for a rectangular wing of aspect ratio 12, using a NACA 4415 airfoil at Reynolds number of 0.75 million from scheme 2. . . . . . . . . . . . . . . . .

4.19 Wing $C_{L^{-}} \alpha$ predicted using schemes 1 and 2 for a rectangular wing of aspect ratio 9, using a NACA 4415 airfoil at Reynolds number of 0.75 million. . . . . . . . . . . . . . . . . . .

4.20 Spanwise $C_{l}$ distribution predicted for a rectangular wing of aspect ratio 9, using a NACA 4415 airfoil at Reynolds number of 0.75 million from scheme 1. . . . . . . . . . . . . . . .

4.21 Spanwise $C_{l}$ distribution predicted for a rectangular wing of aspect ratio 9, using a NACA 4415 airfoil at Reynolds number of 0.75 million from scheme 2. . . . . . . . . . . . . . . 
4.22 Wing $C_{L^{-}} \alpha$ predicted using scheme 2 for a rectangular wing of aspect ratio 6, using a NACA 4415 airfoil at Reynolds number of 0.75 million.

4.23 Spanwise $C_{l}$ distribution predicted for a rectangular wing of aspect ratio 6, using a NACA 4415 airfoil at Reynolds number of 0.75 million from scheme 2. . . . . . . . . . . . . . .

4.24 Wing $C_{L^{-}} \alpha$ predicted from VLM3D for rectangular wings of aspect ratios 12, 9 and 6 using a NACA 4415 airfoil at Reynolds number of 0.5 million. . . . . . . . . . . . . . . . . . . . . . .

4.25 Wing $C_{L^{-}} \alpha$ predicted from experiment for rectangular wings of aspect ratios 12, 9 and 6 using a NACA 4415 airfoil at Reynolds number of 0.5 million. . . . . . . . . . . . . . . . 55

4.26 Planform of the rectangular wing (RHS shown) used in sec. 4.2. . 57

4.27 Planform of the tapered wings (RHS shown) used in sec. 4.2. . . . 58

4.28 Airfoil lift curve used in sec. $4.2-4.5$. . . . . . . . . . . . . 59

4.29 Wing $C_{L^{-}} \alpha$ for wings of different taper ratios, each of aspect ratio 10 using scheme 2. . . . . . . . . . . . . . . . . .

4.30 Change in the spanwise section $C_{l}$ distribution with taper at $\alpha$ of 10 deg using scheme 2. . . . . . . . . . . . . . . .

4.31 Change in the spanwise section $C_{l}$ distribution with taper at $\alpha$ of $17 \mathrm{deg}$ using scheme 2. . . . . . . . . . . . . . . .

4.32 Change in the spanwise section $C_{l}$ distribution with taper at $\alpha$ of 18 deg using scheme 2. . . . . . . . . . . . . . .

4.33 Change in the spanwise section $C_{l}$ distribution with taper at $\alpha$ of 24 deg using scheme 2. . . . . . . . . . . . . . . . . . . 62

4.34 Planform of the part-tapered wing (RHS shown). . . . . . . . . 62

4.35 Lift curves for the part-tapered wing. . . . . . . . . . . . 63

4.36 Spanwise $C_{l}$ distributions for the part-tapered wing. . . . . . . . . 64

4.37 Planform of the wing-tail configuration (RHS shown). . . . . . 65

4.38 Lift curves for the wing-tail configuration. . . . . . . . . . . 66

4.39 Individual contributions of the wing and tail to the total lift of the wing-tail configuration. . . . . . . . . . . . 67

4.40 Spanwise $C_{l}$ distributions for the wing-tail configuration. . . . . . 68

4.41 Pitching-moment curve for the wing-tail configuration. . . . . . . 69

4.42 Planform of the wing-canard configuration (RHS shown). . . . . . 70

4.43 Lift curves of the wing-canard configuration. . . . . . . . . . . . 71

4.44 Spanwise $C_{l}$ distributions for the wing-canard configuration. . . . 72

4.45 Individual contributions of the wing and canard to the total lift of the wing-canard configuration. . . . . . . . . . . 73

4.46 Initial $\delta_{1}$ distribution along wing span. . . . . . . . . . . . . . 74

4.47 Wing $C_{L}$ with an initial asymmetric distribution of $\delta_{1}$. . . . . . 75

4.48 Final converged spanwise section $C_{l}$ with an initial asymmetric dis-

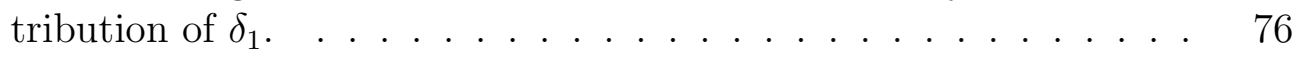


4.49 Spanwise section $C_{l}$ with an initial asymmetric distribution of $\delta_{1}$ after 1 iteration. . . . . . . . . . . . . . 77

4.50 Spanwise section $C_{l}$ with an initial asymmetric distribution of $\delta_{1}$ after 100 iterations. . . . . . . . . . . . . . 77

4.51 Final converged spanwise $\delta_{1}$ distributions. . . . . . . . . . . . 78

4.52 Spanwise $\delta_{1}$ distributions after 1 iteration. . . . . . . . . . . . 79

4.53 Spanwise $\delta_{1}$ distributions after 100 iterations. . . . . . . . . . 79

4.54 Spanwise distributions of $\alpha_{\text {eff }}$ after 1 iteration. . . . . . . . . . . 80

4.55 Spanwise distributions of $\alpha_{\text {eff }}$ after 100 iterations. . . . . . . . . . 80

4.56 Final converged spanwise distributions of $\alpha_{\text {eff }} \ldots \ldots . . . . . .81$

A.1 Unit normal to a lattice. . . . . . . . . . . . . . . . 89

A.2 Horse-shoe vortex at the trailing edge. . . . . . . . . . . . . 91

A.3 Flow chart of VLM3D. . . . . . . . . . . . . . . . . . . . 94 


\section{Nomenclature}

C damping factor

$C_{L} \quad$ wing lift coefficient

$C_{l} \quad$ airfoil lift coefficient

$C_{m} \quad$ airfoil pitching moment coefficient about the quarter chord

c chord

F residual vector

$f \quad$ element of residual vector

$i, j \quad$ index of wing section

J Jacobian matrix

LLT lifting line theory

$N \quad$ number of wing sections

$p \quad$ perturbation to $\delta_{1}$ or $\delta_{2}$

VLM vortex lattice method

$x_{2} \quad$ chordwise start location of the second decambering function

$\alpha \quad$ angle of attack 
$\beta \quad$ angle of yaw

$\Gamma \quad$ strength of bound vortex

$\delta \mathbf{x} \quad$ vector containing the corrections to the Newton variables

$\delta_{1}(x)$ first decambering function

$\delta_{2}(x)$ second decambering function

$\theta_{2}(x)$ angular coordinate corresponding to $x_{2}$

\section{Subscripts}

1 scheme 1

$2 \quad$ scheme 2

$\max$ maximum

$p \quad$ perturbed value for a given step of the iteration

$s \quad$ starting value for a given step of the iteration

sec represents value for a wing section

$t \quad$ target value for a given step of the iteration

visc represents value from two-dimensional viscous experimental or computational data 


\section{Chapter 1}

\section{Introduction}

The ability of linear aerodynamic methods such as lifting-line theory (LLT), Weissinger's method and vortex-lattice methods to successfully predict the lift and induced drag behavior of medium to high aspect ratio wings at small angles of attack is well established. In these methods, a linear lift-curve slope is assumed for the airfoil sections that form the wings. This lift-curve slope is typically close to $2 \pi$ per radian. For several decades, researchers have sought to extend these linear prediction methods to handle the analysis of wings in which nonlinear airfoil lift curves are taken into consideration. The motivation was provided by the desire to predict stall and post-stall characteristics of wings using experimental or computational section data for these high angles of attack. It is recognised that the flow over a wing at post-stall conditions is highly three dimensional and that considerable error can result in using a "strip-theory" approach. The impetus for such a prediction method, however, is provided by the need for rapid prediction capabilities for such high-alpha conditions for aircraft stability, control and simulation purposes and in the early design phases. Furthermore, even high-fidelity computational fluid dynamics (CFD) techniques are only now approaching the stage where they can be reliably used for high-alpha aerodynamic prediction. These CFD based techniques, however, require large computing resources and significant time even for the analysis at a single angle of attack. There 
are also several additional isues that currently prevent the routine use of CFD for high-alpha predictions such as the time required for generating high-quality grids for each configuration. Thus the search for approximate approaches for stall and post-stall prediction of wings using known section data continues to be of interest.

The approaches for extending the linear aerodynamic prediction methods to handle nonlinear and post-stall airfoil lift curves can be broadly classified into two kinds. In the first approach, a lift distribution is first assumed on the wing, and it is then iteratively corrected by determining the effective- $\alpha$ distribution using the nonlinear airfoil lift curve. In the second approach, the deviation of the airfoil nonlinear lift curve from the potential-flow linear lift curve is used to apply a correction to the local $\alpha$ at each section of the wing.

A literature study of the development of flow prediction methods over the years and brief descriptions of the two approaches follows.

\section{$1.1 \quad$ Literature Study}

With the remarkable success of Prandtl's lifting-line theory (LLT) in being able to predict the flow past medium to high aspect ratio unswept wings in incompressible flow, LLT became a standard tool for computing wing aerodynamics. LLT uses a single unswept lifting line (or bound vortex) to model the circulation on the wing. The strength of this bound vortex, $\Gamma$, varies along the span. At any given spanwise location, the change in $\Gamma$ is shed as trailing vorticity, which in turn causes induced velocities along the lifting line. LLT enables the computation of the $\Gamma$ distribution for which the accompanying induced velocities and the resulting effective angles of attack along the span are consistent with the $\Gamma$ distribution. For this purpose, the classical Prandtl LLT assumes a linear lift-curve slope for the airfoil sections that form the wing. This lift-curve slope is typically close to 
$2 \pi$ per radian. With the success of LLT in the prediction of wing flows at low angles of attack, the attention soon turned to whether LLT could be modified for the analysis of wings where nonlinear lift-curve slopes for the airfoil sections can be taken into consideration. The studies in this regard can be widely divided into two categories as described below:

\subsubsection{The Iterative $\Gamma$ distribution Approach}

Tani $^{1}$ is believed to have developed the first successful technique in 1934 for handling nonlinear section lift-curve slopes in the LLT formulation. In his technique, a spanwise bound vorticity $(\Gamma)$ distribution is first assumed; this distribution is used to compute the distribution of induced velocities and hence induced angles of attack and effective angles of attack along the lifting line. The distribution of the effective angles of attack is then used to look up the operating $C_{l}$ of the local section using the known nonlinear $C_{l^{-}} \alpha$ data for the airfoil. A new $\Gamma$ distribution is then computed from the spanwise $C_{l}$ distribution. The iteration is carried out till the $\Gamma$ distribution converges. This method worked well up to the onset of stall and was made popular by the NACA report of Sivells and Neely ${ }^{2}$ in 1947 that provided a detailed description of the method and implemented a tabular procedure for hand-calculation of the method for unswept wings with arbitrary planform and airfoil lift-curve slopes. This method was applied for analysis of wings up to the onset of stall, i.e. until a wing angle of attack at which some section on the wing has $C_{l}$ equal to the local section $C_{l \max }$. At higher angles of attack where some sections on the wing may have a negative lift-curve slope, this successive-approximation approach appears to have failed.

Extension of the approach to post-stall angles of attack have been investigated in several subsequent research efforts. Based on a suggestion by von Kármán, Schairer ${ }^{3}$ and Sears ${ }^{4}$ investigated the possibility of non-unique solutions for post- 
stall angles of attack. According to Sears, ${ }^{4}$ von Kármán noticed that Prandtl's lifting-line equation has non unique solutions for cases when the lift-curve slope becomes negative (i.e. when the $\alpha$ increases past the onset of stall). These non unique solutions include both symmetric and antisymmetric lift distributions even when both the geometry and onset flow are symmetric. Sears ${ }^{4}$ mentions that von Kármán further postulated that even in the conditions just past the onset of wing stall, when some sections of the wing may have positive lift-curve slopes (prestall condition) and other sections may have negative lift-curve slopes (post-stall condition), non unique and asymmetric lift distributions are possible. It occurred to von Kármán and Sears that the appearance of large and sometimes violent rolling moments past stall on symmetric wind-tunnel models at zero yaw may be explained by the possibility of asymmetric lift distributions at perfectly symmetric flight conditions.

The investigation suggested by von Kármán on computing the symmetric and asymmetric lift distributions on wings operating beyond stall was carried out and was reported in a 1939 thesis $^{3}$ by Schairer working under the supervision of Sears. Schairer apparently used the same procedure as that pioneered by Tani, but had to use a tedious trial-and-error procedure to find the solutions as Tani's successiveapproximation procedure failed to work for these partially-stalled cases. Sears ${ }^{4}$ presents some of Schairer's results for a flat, untwisted elliptic wing of AR 10.19 operating beyond stall. Their research showed that the multiple solutions for poststall angles of attack include asymmetric lift distributions even when the flight condition and geometry are perfectly symmetrical. The results show solutions consisting of asymmetric lift distributions (in addition to a classical symmetric solution) with large associated rolling moments for a narrow range of angles of attack just beyond stall. Sears mentions that the choice between the symmetric and asymmetrical solution would require the formidable solution of the relative 
stability of the two flows. Sears concludes by pointing out the need for further progress on the analysis of wings at near- and post-stall conditions.

Piszkin and Levinsky ${ }^{5}$ developed a nonlinear lifting line method based in part on the iterative method originally conceived by Tani. ${ }^{1}$ As described in Ref. 6, they were motivated by the need for a method that could predict, simulate, and alleviate adverse wing stalling characteristics such as wing drop, loss of roll control and roll control reversal at zero yaw. These characteristics were believed to be caused by the occurrence of asymmetric lift distributions on wings with stalled or partially-stalled flow.

The method of Piszkin and Levinsky utilizes a finite element, unsteady wake, incompressible flow theory that can be used for analysis at either zero or nonzero yaw. The model uses a single chordwise row of horseshoe vortices distributed along the span, with the bound vortex aligned with the local quarter-chord line. The boundary condition of zero normal flow is applied at the control point, which is the three-quarter-chord location for each horseshoe vortex. As a consequence of using a single chordwise horseshoe vortex, the method is restricted to wings of moderate to high aspect ratio. Although Levinsky refers to the method as a lifting-line method, the vortex model is more commonly referred to as a vortex lattice method (with a single chordwise row of horseshoe vortices) or a discretevortex Weissinger's method. It must be mentioned that this method differs from Prandtl's classical LLT in the implementation of the boundary condition.

In the method of Piszkin and Levinsky, at each step of the iteration, the downwash computed using the $\Gamma$ distribution from the previous time step is used to compute the change in the $\Gamma$ distribution using the airfoil lift curve. This change, multiplied by a specified damping factor, $C$, is then added to the old $\Gamma$ distribution to obtain the new $\Gamma$ distribution for the next iteration. A damping factor of $C<1$ is required to stabilize the iterations, although it results in a larger 
number of iterations for convergence. Unlike in the traditional LLT, where the distribution of the effective section angles of attack is computed as part of the solution, with the vortex model that Piszkin and Levinsky used in their method, the distribution of the effective section angles of attack is not readily available. They have, however, bypassed this difficulty by defining the effective downwash angle at a section as $\alpha_{3 D}-\alpha_{2 D}$, where $\alpha_{3 D}$ is the downwash angle at the control point resulting from the entire vortex system and $\alpha_{2 D}$ is the induced angle from an infinite span bound vortex along the $c / 4$ line with strength equal to that of the horseshoe vortex under consideration. From this downwash angle, they compute the effective angle of attack at every section of the wing. This formulation does not include the effects of sweep and dihedral for the effective angle of attack. ${ }^{6}$ Using their method, Piszkin and Levinsky found that multiple converged solutions are possible, including some that have saw-tooth type oscillations in the spanwise lift distributions. Because they were restricted to the use of 10 panels per side of the wing in their computer program, they were unable to determine whether these oscillations are present for more dense panel distributions. To avoid these oscillations, they used a switching logic that restarts the iteration procedure with an initial distribution having a zero induced $\alpha$ for any wing section found to be stalled.

With this method, the effects of different wing planform shapes and airfoil lift curves were investigated. Piszkin and Levinsky present the occurrence of lift hysteresis for increasing and decreasing $\alpha$ and the occurrence of zero- $\beta$ rolling moments at post-stall conditions. The results confirm that depending on the starting solution for the initial lift distribution for the iteration, multiple solutions are possible for the converged lift distribution for a post-stall angle of attack. Some of these lift distributions may be asymmetric even though the flight condition is exactly symmetric. The asymmetric solutions for zero $\beta$ were obtained by using 
a converged solution for a nonzero $\beta$ as a starting point for the iteration. Like Sears, Levinsky ${ }^{6}$ also points out the need for a method of calculating the relative stability of the different possible solutions for the lift distribution at a given angle of attack. Finally, Levinsky ${ }^{6}$ points out the need for an unsteady nonlinear lifting surface theory that can handle low aspect ratio wings for fighters and other such configurations since until that point nonlinear methods were capable of handling only moderate to high aspect ratio wings. The Piszkin-Levinsky method has recently been used by Anderson ${ }^{7}$ for aircraft high- $\alpha$ stability analysis.

Four years after Levinsky's publication, Anderson, Corda, and Van Wie ${ }^{8}$ published a nonlinear lifting-line theory that they applied to drooped leading-edge wings below and above stall. At that time, there was considerable interest in improving the stall-spin behavior of general aviation aircraft, and part-span drooped leading-edge wings were generating interest for their benign stall characteristics. Their article provides guidelines for the design of such wings and presents results for $C_{L^{-}} \alpha$ curves that extended to very high post-stall angles of attack close to 50 deg.

McCormick presents a similar, independently developed approach ${ }^{9}$ wherein the nonlinear lifting-line theory was used to examine the loss in roll damping for a wing near stall. In both Refs. 8 and 9, researchers reported that no asymmetric lift distributions for symmetric flight conditions are observed even when the iterations were started with asymmetric initial lift distribution. This observation differs from those of Sears and Levinsky.

\subsubsection{The $\alpha$ Correction Approach}

An entirely different approach to the use of nonlinear section data was developed by Tseng and Lan. ${ }^{10}$ While their main focus was on vortex-dominated flows on low aspect ratio fighter-type wings at high $\alpha$, they incorporate the effect of boundary- 
layer separation by iteratively reducing the angle of attack at each section of the wing. The reduction at any given wing section is determined by the difference between the potential flow solution and the viscous $C_{l}$ from the nonlinear section $C_{l^{-}} \alpha$ curve.

More recently, an approach similar to that reported in Ref. 10 was used by van Dam, Vander Kam and Paris ${ }^{11}$ for rapid estimation of $C_{L \max }$ and other high-lift characteristics for airplane configurations. In their method to estimate $C_{\text {Lmax }}$, at least two airfoil data sets for the root and the tips are required for analysis. If there is significant variation in the spanwise wing geometry, then airfoil data for additional sections along the span are used. Using the sectional lift curves, the slope of the lift curve and zero lift angle of attack are calculated for each section along the wing span. Hence, the initial $C_{l}$ distribution along span is calculated. A complete sweep of angles of attack is then performed and at each angle of attack, an iterative procedure is employed to improve upon the initial spanwise $C_{l}$ distribution. The local effective angle of attack is calculated by adding a correction for viscous flow. If the difference between the viscous and linear potential section $C_{l}$ at a section is greater than a given tolerance then the section angle of attack is corrected by a factor of the difference in the viscous and potential $C_{l}$ to the lift curve slope calculated earlier. Having made corrections to the distribution of $\alpha$ along the wing span, the modified $C_{l}$ distribution is calculated. This process is repeated till the difference in the viscous and potential $C_{l}$ values at all spanwise sections is within a given tolerance. In Ref. 11, this method was used primarily for the estimation of $C_{L \max }$ and not for post-stall analysis. 


\subsection{Current Approach}

In the current research effort, a decambering approach (Scheme 1) was developed ${ }^{12}$ for predicting post-stall aerodynamic characteristics of wings using known section data. In this approach, the chordwise camber distribution at each section of the wing is reduced to account for the viscous effects at high angles of attack. This approach is somewhat similar to that developed in Ref. 10, but differs in its capability to use both the $C_{l}$ and the $C_{m}$ data for the section and in the use of a two-variable function for the decambering. In addition, unlike all of the earlier methods, the current approach uses a multidimensional Newton iteration that accounts for the cross-coupling effects between the sections in predicting the decambering for each step in the iteration. This decambering approach was applied to the post-stall prediction of single wings in Ref. 12 .

However, it was found that Scheme 1 worked for only some airfoil lift curves. Therefore, Scheme 2 was developed ${ }^{13}$ based on the same approach but differed in the way the residuals are computed. Scheme 2 is found to be more robust than Scheme 1 and works for several airfoil lift curves.

Chapter 2 describes the decambering approach for the flow past an airfoil. Chapter 3 describes the incorporation of the decambering approach into the poststall analysis of a three-dimensional wing. Chapter 4 presents results to validate the use of the decambering approach for post-stall prediction purposes. Conclusions are drawn in Chapter 5. Possible future work is discussed in Chapter 6 . Appendix A provides a detailed description of VLM3D, which is the code developed to implement the decambering approach in a vortex lattice method, for post-stall prediction puposes. 


\section{Chapter 2}

\section{Illustration of the Decambering Concept for Flow Past an Airfoil}

The overall objective of the current research is to arrive at a scheme for incorporating the nonlinear section lift curves in wing analysis methods such as Lifting Line Theory (LLT), discrete-vortex Weissinger's method and vortex lattice methods (VLM). For this purpose the wing span is assumed to consist of several sections and for each of these sections it is assumed that the two-dimensional data $\left(C_{l^{-}} \alpha\right.$ and $\left.C_{m}-\alpha\right)$ is available from either experimental or computational results.

Nonlinear lift curve slopes in wing analysis are incorporated by finding the effective angle of attack, $\alpha_{e f f}$, and the corresponding effective reduction in camber at each section of the wing. The effective reduction in camber or "decambering" is determined iteratively. The decambering at each section is modeled using a function of two variables.

In the following sections in this chapter, the concept of "decambering" is first discussed for a two-dimensional airfoil. The procedure for calculating the twovariable decambering function and the use of this decambering function to account for the differences in the potential and viscous flows for an airfoil is explained in

detail. Results are then presented to show the effectiveness of the decambering approach for an airfoil. Having introduced the decambering concept for an airfoil 
in this chapter, its incorporation into the post-stall analysis of a three-dimensional wing is then discussed in Chapter3.

\subsection{The Decambering Concept}

This section illustrates the concept of decambering by using a simple example of a two-dimensional flow past a NACA 0012 airfoil. With increasing angle of attack, the boundary layer thickens on the upper surface and finally separates, as shown in Fig. 2.1. It is this flow separation that causes the viscous results for $C_{l}$ and $C_{m}$ to deviate from the predictions obtained using potential-flow theory. The reason for the deviation can be related to the effective change in the airfoil camber distribution due to the boundary-layer separation. If the decambering is taken into consideration, then a potential-flow prediction for the decambered airfoil will closely match the viscous $C_{l}$ and $C_{m}$ for the high- $\alpha$ flow past the original airfoil shape. This decambering idea served as the basis for the formulation of the current approach for the three-dimensional flow problem.

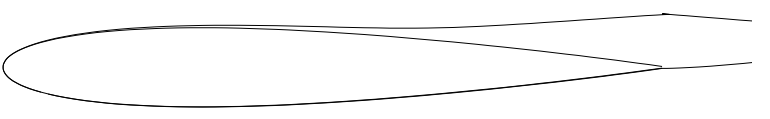

Figure 2.1: Flow separation from an airfoil at a high angle of attack.

While the camber reduction due to the flow separation can be determined from computational flows, no such detailed information is available from wind tunnel results that typically provide only the $C_{l^{-}} \alpha$ and $C_{m^{-}} \alpha$ curves. This section discusses the approach for determining an "equivalent" camber reduction from $C_{l^{-}} \alpha$ and $C_{m^{-}} \alpha$ curves for an airfoil. More specifically, the effective decambering for an $\alpha$ is computed using the deviations of the viscous $C_{l}$ and $C_{m}$ from the potential flow predictions for that airfoil. 


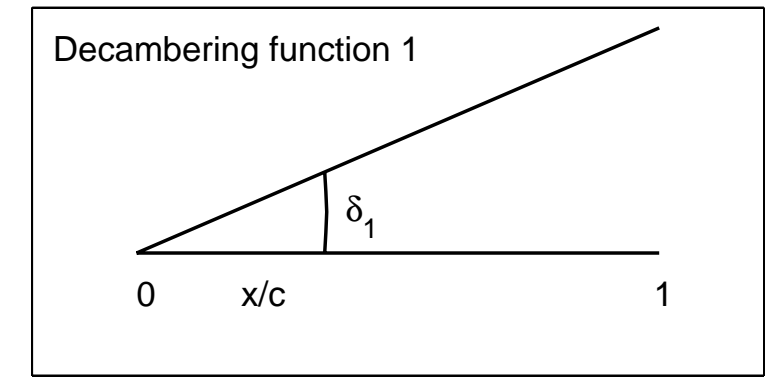

(a)

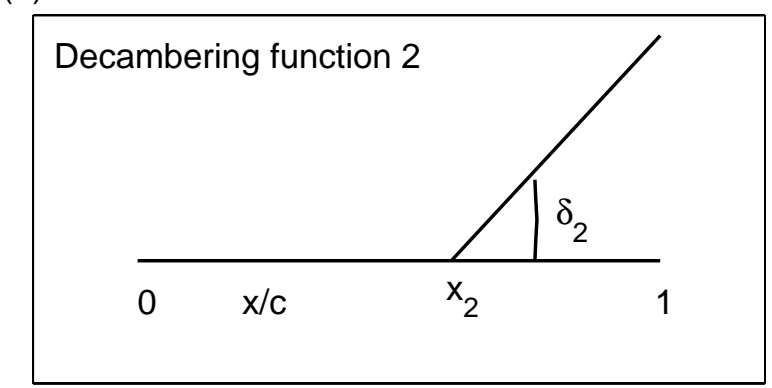

(b)

Figure 2.2: Schematic diagram of functions 1 and $2\left(\delta_{1}\right.$ and $\delta_{2}$ are negative as shown) used to model effective decambering of an airfoil.

In the current method, the effective decambering for an airfoil is approximated using a function of two variables $\delta_{1}$ and $\delta_{2}$. The two linear functions shown in Fig. 2.2 are superposed to obtain the final decambering function. Two variables are used because the decambering is determined using two pieces of information: the $C_{l}$ and $C_{m}$ from the airfoil data for the $\alpha$ under consideration. This approximation will, of course, not match the actual viscous decambering, but the objective is to find an equivalent camber reduction to match the viscous $C_{l}$ and $C_{m}$ for the $\alpha$ under consideration.

For the illustration of the decambering for two-dimensional flow over an airfoil, the following procedure is used:

1. Determine the viscous $C_{l}$ and $C_{m}$ for the $\alpha$ under consideration from experimental or computational data for the airfoil.

2. Compute the corresponding potential-flow $C_{l}$ and $C_{m}$ using a lumped vortex model of the actual camberline of the airfoil. Based on an empirical 

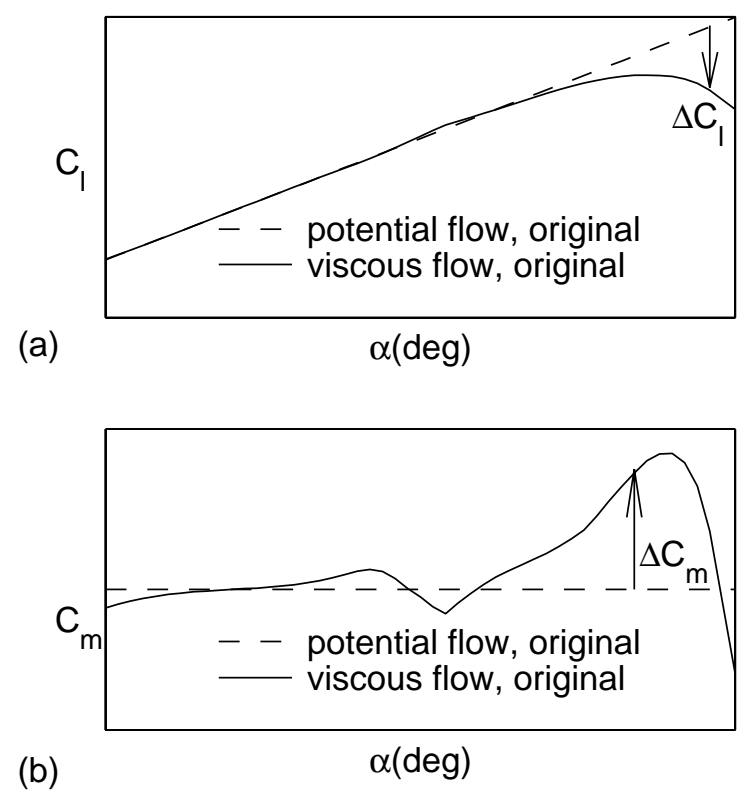

Figure 2.3: $C_{l}$ and $C_{m}$ of the NACA 0012 airfoil.

approach of Katz and Plotkin, ${ }^{14}$ a thickness correction is applied to the $C_{l}$ by considering the $C_{l}$ times the factor $(1+0.77 t / c)$ where $t / c$ is the maximum thickness-to-chord ratio of the airfoil.

3. Compute the difference between the viscous and the potential-flow results: $\Delta C_{l}=\left(C_{l}\right)_{v i s c}-\left(C_{l}\right)_{\text {potential }}$ and $\Delta C_{m}=\left(C_{m}\right)_{\text {visc }}-\left(C_{m}\right)_{\text {potential }}$.

These differences are shown schematically in Fig. 2.3 for an NACA 0012 airfoil analyzed using the XFOIL code. ${ }^{15}$

4. Use the differences $\Delta C_{l}$ and $\Delta C_{m}$ between the viscous and the potentialflow $C_{l}$ and $C_{m}$ respectively to calculate the values of $\delta_{1}$ and $\delta_{2}$. Details of this calculation follow:

The effects of changing $\delta_{1}$ and $\delta_{2}$ on the $C_{l}$ and $C_{m}$ for a given $\alpha$ can be computed reasonably well using thin airfoil theory and a three-term Fourier series approximation for a flat plate with a flap deflection. ${ }^{14}$ The values of $\delta_{2}$ and $\delta_{1}$ in radians for a given $\Delta C_{l}$ and $\Delta C_{m}$ have been derived and are presented in Eqs. 2.1 and 2.2 respectively. In these equations, $\theta_{2}$ is the angular location in radians of 


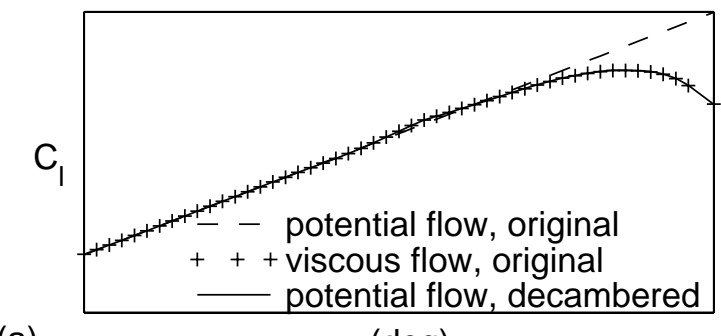

(a)

$\alpha(\operatorname{deg})$

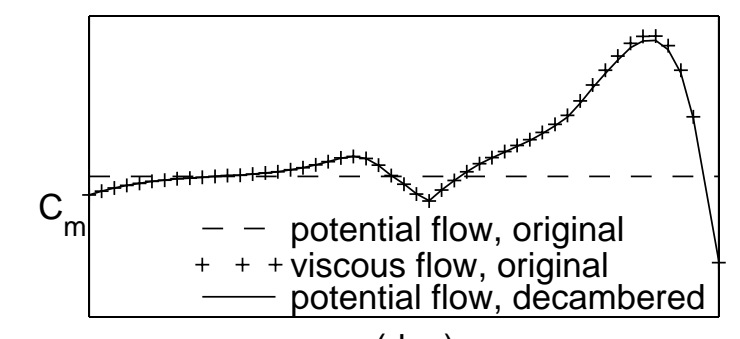

(b)

$\alpha(\operatorname{deg})$

Figure 2.4: $C_{l}$ and $C_{m}$ using potential-flow with decambering.

the start point for the second decambering function shown in Fig. 2.2(b) and can be expressed in terms of its $x$-location, denoted by $x_{2}$, as shown in Eqn. 2.3. In the current work, $x_{2} / c$ is arbitrarily assumed to be 0.8 , although any value from 0.5 to 0.9 typically works well. The overall approach used for this illustration is shown as a flow chart in Fig. 2.9.

$$
\begin{gathered}
\delta_{2}=\frac{\Delta C_{m}}{\frac{1}{4} \sin 2 \theta_{2}-\frac{1}{2} \sin \theta_{2}} \\
\delta_{1}=\frac{\Delta C_{l}-\left[2\left(\pi-\theta_{2}\right)+2 \sin \theta_{2}\right] \delta_{2}}{2 \pi} \\
\theta_{2}=\cos ^{-1}\left(1-2 x_{2} / c\right) ; x_{2} / c=0.8
\end{gathered}
$$

To verify the effectiveness of the decambering approach, the values of $\delta_{1}$ and $\delta_{2}$ were calculated for the viscous $C_{l^{-}} \alpha$ and $C_{m^{-}} \alpha$ data shown for the NACA 0012 airfoil in Fig. 2.3. These values were then applied as corrections to the flat-plate 


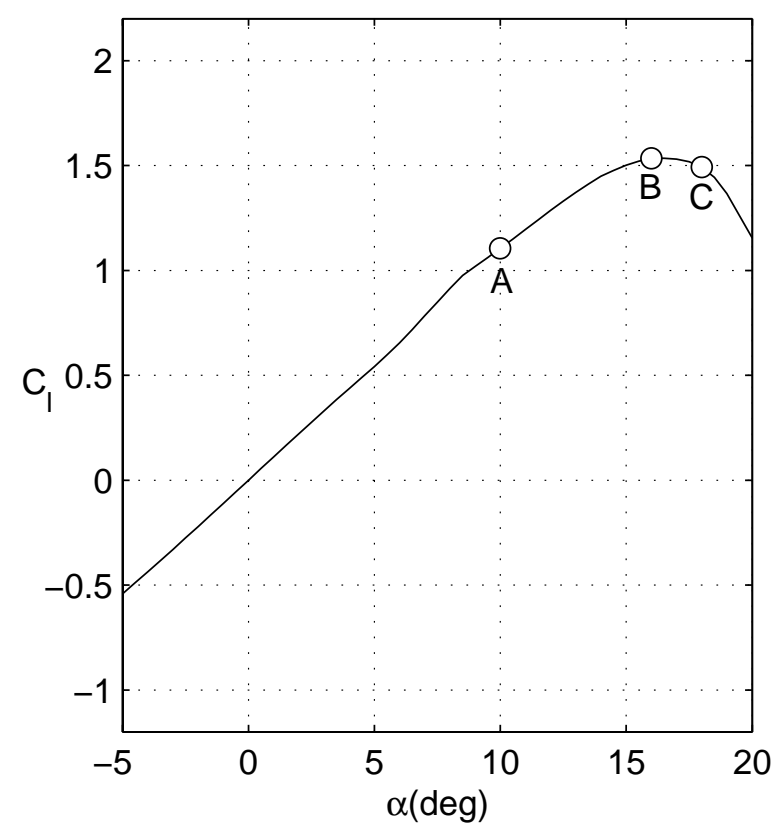

Figure 2.5: The $\alpha$ s chosen to illustrate effectiveness of decambering.

camberline for potential-flow analysis of the NACA 0012 airfoil using a lumped vortex method. ${ }^{14}$ Figure 2.4 shows for comparison the predicted potential-flow $C_{l^{-}} \alpha$ and $C_{m^{-}} \alpha$ curves for the decambered airfoil with the viscous result from XFOIL analysis. The agreement is seen to be very good, which confirmed that the two-variable decambering function can be used to model nonlinear lift as well as pitching moment curves for high angles of attack.

To compare the approximate decambering from the two-variable function with the actual decambering from the viscous solution, comparison plots have been made for $\alpha$ of 10, 16 and 18 deg for the NACA 0012 example which are shown as $\mathrm{A}, \mathrm{B}$ and $\mathrm{C}$ respectively in Fig. 2.5. The actual decambering was computed from the boundary-layer displacement thickness distributions predicted by XFOIL. Figures $2.6,2.7$, and 2.8 show (a) the airfoil geometry with the boundary-layer overlay and (b) the approximate and the actual decambering functions for the three angles of attack. The progressive increase in the decambering required to model the boundary-layer separation at higher angles of attack is observed from these 
figures. Also observed is that the two-variable function used provides a reasonable approximation of the actual decambering of the airfoil.

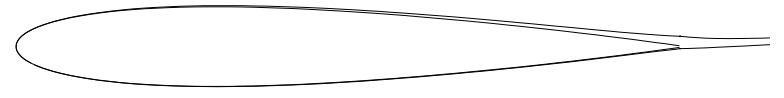

(a) NACA 0012 airfoil with boundary layer at $\alpha$ of $10 \mathrm{deg}$.

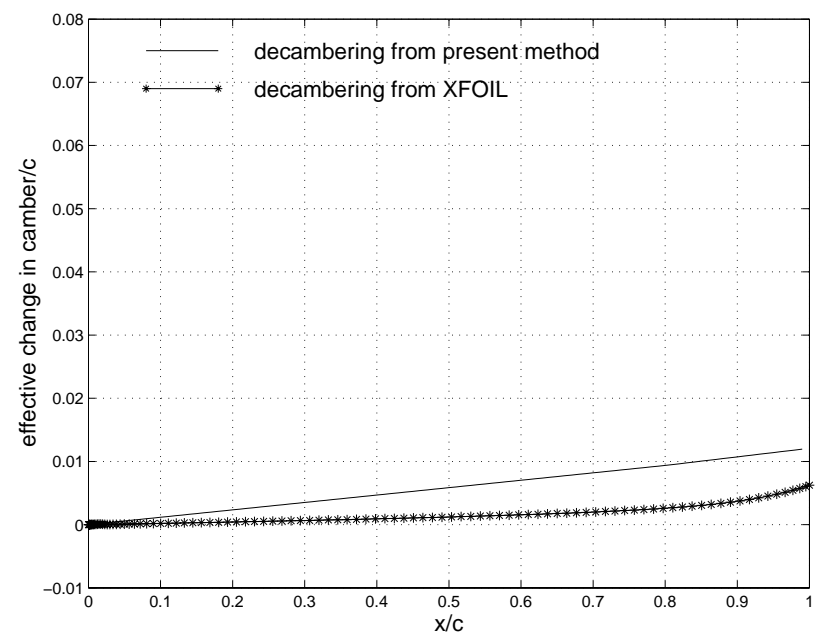

(b) Decambering function and XFOIL result.

Figure 2.6: Effectiveness of the decambering for $\alpha$ of $10 \mathrm{deg}$. 


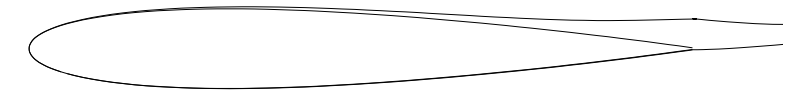

(a) NACA 0012 airfoil with boundary layer at $\alpha$ of $16 \mathrm{deg}$.

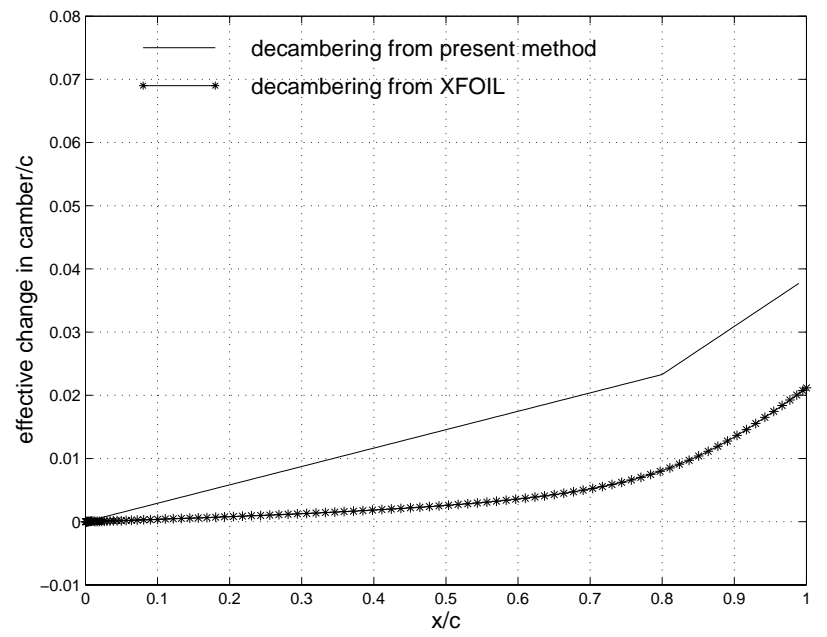

(b) Decambering function compared and result.

Figure 2.7: Effectiveness of the decambering for $\alpha$ of $16 \mathrm{deg}$. 


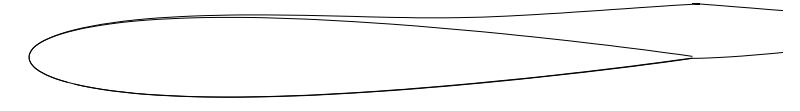

(a) NACA 0012 airfoil with boundary layer at $\alpha$ of $18 \mathrm{deg}$.

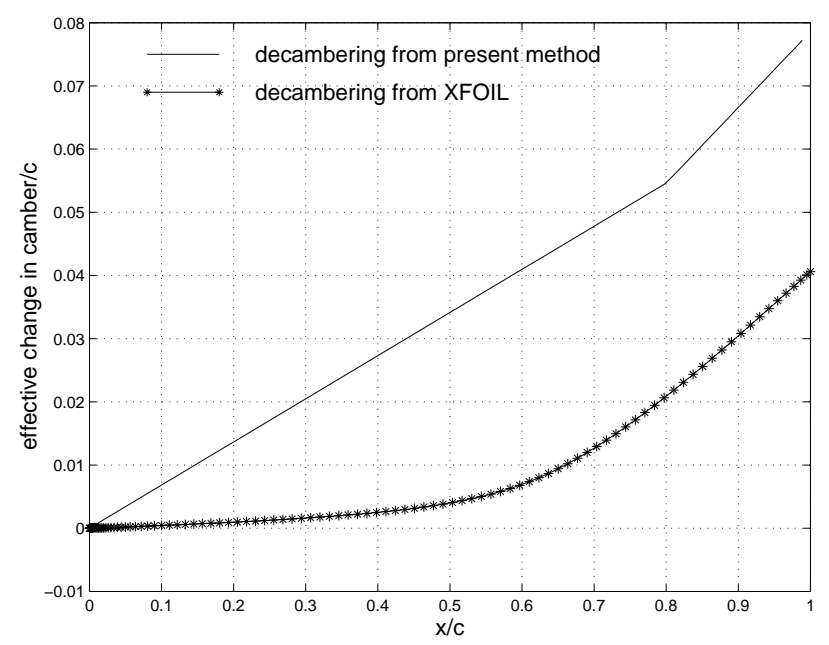

(b) Decambering function compared and XFOIL result.

Figure 2.8: Effectiveness of the decambering for $\alpha$ of $18 \mathrm{deg}$. 


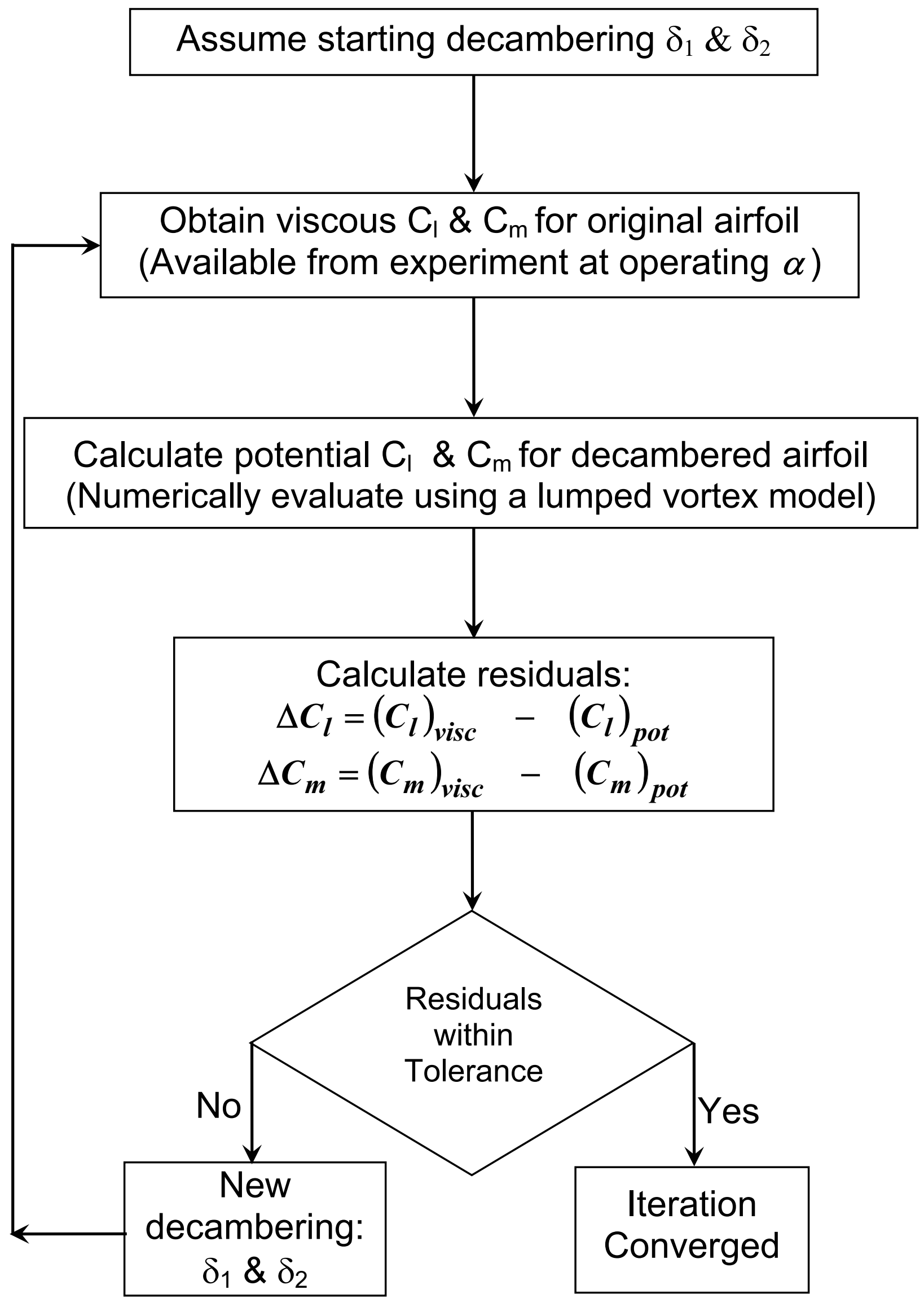

Figure 2.9: Flow chart of the iterative decambering approach in 2D flow. 


\section{Chapter 3}

\section{Post-stall prediction of a finite wing}

Using the overall methodology described in chapter 2, two schemes have been formulated for determining the post-stall solution of a finite wing. The primary difference between the two schemes is in the details of how the residuals, $\Delta C_{l}$ and $\Delta C_{m}$, are computed at each section of the wing. The first scheme, introduced in Ref. 12, was found to work well for certain airfoil lift curves, but failed to converge for several other airfoil lift-curves. This lack of robustness provided the impetus for developing the second scheme. The following sections explain the decambering approach for a wing, the overall iteration procedure used to implement the decambering approach, and the two schemes in detail.

\subsection{Decambering for a wing}

The objective of the current research was to incorporate the two-variable decambering function described in Chapter 2 for the analysis of a wing. For this purpose, a vortex lattice method (VLM) is used and the values of $\delta_{1}$ and $\delta_{2}$ at each section of a wing(s) are evaluated in an iterative fashion. 


\subsubsection{Vortex Lattice Method(VLM)}

In a typical VLM, the lifting surface is divided into several spanwise and chordwise lattices. Associated with each of these lattices is a ring vortex as shown in Fig. 3.1. The primary advantage of using ring vortex elements is that they can be easily implemented in a computer program. Also, the zero-normal-flow boundary condition is satisfied on the actual lifting surface which may have camber and different planform shapes. In the current work unsteady analysis is not done. Therefore, the wake behind the wing is not discretized and in order to satisfy the Kutta condition at the trailing edge the wake is replaced by a series of horse-shoe vortices. The leading segment of the vortex ring is placed on the lattice's quarter chord line and the control point is at the center of the three-quarter chord line of the lattice. The zero-normal-flow boundary condition is satisfied at the control point of each lattice. A positive $\Gamma$ is defined according to the right-hand rotation rule. Inthe current work, the VLM3D code was developed for analysis of multiple-lifting-surface configurations and the decambering approach was also implemented. A complete detailed description of the VLM3D code is provided in Appendix A.

\subsubsection{Predicting the decambering along wing span}

As explained earlier, the lifting surface is divided into several spanwise and chordwise lattices. Each spanwise section $j$ (composed of a row of chord-wise lattices) has two variables, $\delta_{1 j}$ and $\delta_{2 j}$, for defining the local decambered geometry at that section. Unlike in the two-dimensional case, where the $\delta_{1}$ and $\delta_{2}$ are selected to match the differences between the potential-flow and the viscous-flow results, in the three-dimensional case, changing a $\delta$ on one section is likely to have a significant effect on the neighboring sections and on the sections of the downstream 


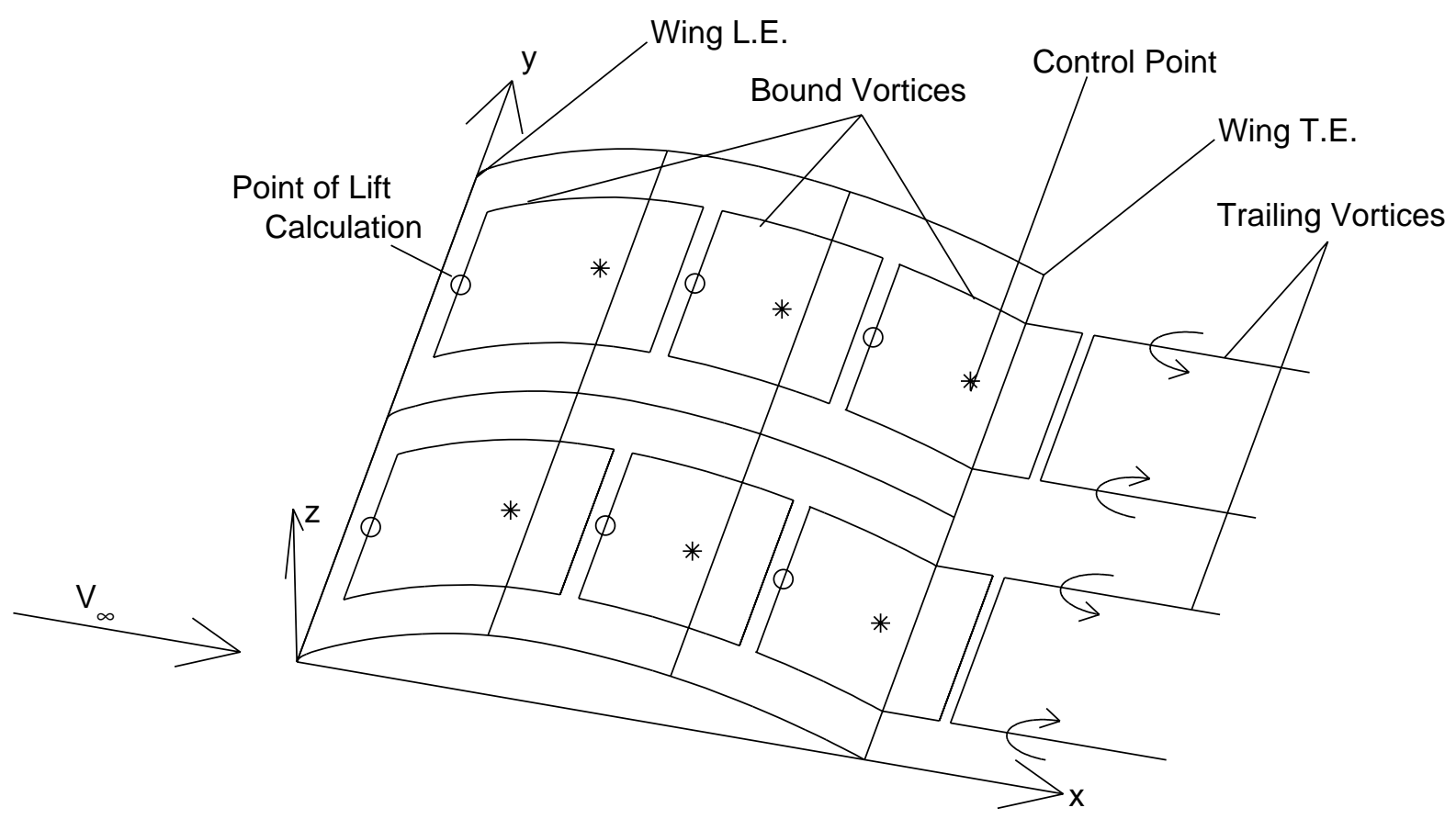

Figure 3.1: Vortex Lattice Method(only six lattices shown for clarity).

lifting surfaces. To account for these effects, a $2 N$-dimensional Newton iteration is used to predict the $\delta_{1}$ and $\delta_{2}$ at each of the $N$ sections of all the wings so that the $\Delta C_{l}$ and $\Delta C_{m}$ at these sections approach zero as the iteration progresses. A $2 N \times 2 N$ matrix equation as shown in Eqn. 3.1 has to be solved for each step of the Newton iteration. ${ }^{16}$ In this equation, $\mathbf{F}$ is a $2 N$-dimensional vector containing the residuals of the functions $f_{i}$ to be zeroed, $\delta \mathbf{x}$ is the $2 N$-dimensional vector containing the corrections required to the $2 N$ variables $x_{i}$ to bring the vector $\mathbf{F}$ closer to zero, and $\mathbf{J}$ is the $2 N \times 2 N$ Jacobian of the system containing the gradient information.

$$
\mathbf{J} \cdot \delta \mathbf{x}=-\mathbf{F}
$$

The Jacobian is partitioned into four sub-matrices as shown in Eqn. 3.2. Equations 3.3-3.6 show the elements of the four sub-matrices. 


$$
\begin{gathered}
J=\left(\begin{array}{cc}
J_{l 1} & J_{l 2} \\
J_{m 1} & J_{m 2}
\end{array}\right) \\
\left(J_{l 1}\right)_{i, j}=\frac{\partial \Delta C_{l i}}{\partial \delta_{1, j}}=\frac{\left(C_{l p}\right)_{i}-\left(C_{l s}\right)_{i}}{\left[\left(\delta_{1 s}\right)_{j}+p\right]-\left(\delta_{1 s}\right)_{j}} \\
\left(J_{m 1}\right)_{i, j}=\frac{\partial \Delta C_{m i}}{\partial \delta_{1, j}}=\frac{\left(C_{m p}\right)_{i}-\left(C_{m s}\right)_{i}}{\left[\left(\delta_{1 s}\right)_{j}+p\right]-\left(\delta_{1 s}\right)_{j}} \\
\left(J_{l 2}\right)_{i, j}=\frac{\partial \Delta C_{l i}}{\partial \delta_{2, j}}=\frac{\left(C_{l p}\right)_{i}-\left(C_{l s}\right)_{i}}{\left[\left(\delta_{2 s}\right)_{j}+p\right]-\left(\delta_{2 s}\right)_{j}} \\
\left(J_{m 2}\right)_{i, j}=\frac{\partial \Delta C_{m i}}{\partial \delta_{2, j}}=\frac{\left(C_{m p}\right)_{i}-\left(C_{m s}\right)_{i}}{\left[\left(\delta_{2 s}\right)_{j}+p\right]-\left(\delta_{2 s}\right)_{j}}
\end{gathered}
$$

For each step of the iteration, $\mathbf{F}$ and $\mathbf{J}$ are determined, and $\delta \mathbf{x}$ is computed using Eqn. 3.1. The corrections are then applied to the values of $\delta_{1}$ and $\delta_{2}$ for all the sections in an effort to bring the residuals closer to zero.

\subsection{The iteration procedure}

The iteration scheme can be summarized using the flow chart in Fig. 3.2, the illustration in Fig. 3.3 and the following procedure:

1. Assume starting values of the decambering defined by $\delta_{1}$ and $\delta_{2}$ for each section of the wing; for example, section $j$ has starting values denoted by $\left(\delta_{1 s}\right)_{j}$ and $\left(\delta_{2 s}\right)_{j}$;

2. Compute the wing aerodynamic characteristics using VLM3D. For this computation, the unit normal vector of each lattice is rotated to account for the decambered shape of each section of the wing; the VLM3D analysis provides the $C_{l}$ and $C_{m}$ of each section as output. These are the starting values for 
the current step of the iteration and are denoted by $\left(C_{l_{s}}\right)_{j}$ and $\left(C_{m s}\right)_{j}$ for section $j$.

3. Compute the starting values of the local effective angles of attack of each section corresponding to the section $C_{l}$ of that section; for example, the local effective angle of attack of section $j$ is obtained by setting $\left(C_{l}\right)_{\text {sec }}=$ $\left(C_{l_{s}}\right)_{j}$ in Eqn. 3.7. Let this effective angle of attack be denoted by $\left(\alpha_{s}\right)_{j}$. Eqn. 3.7 assumes a section lift-curve slope of $2 \pi$ per radian and accounts for the zero-lift angle of attack of the decambered section, which depends on the values of $\delta_{1}$ and $\delta_{2}$ and the $\alpha_{0 l}$ of the original airfoil camberline.

$$
\alpha_{e f f}=\frac{\left(C_{l}\right)_{s e c}}{2 \pi}-\delta_{1}-\delta_{2}\left[1-\frac{\theta_{2}}{\pi}+\frac{\sin \theta_{2}}{\pi}\right]+\alpha_{0 l}
$$

4. Residuals for Scheme 1: Compute the target $C_{l}$ of each section; for example, the target $C_{l}$ of section $j$ is given by $\left(C_{l t, 1}\right)_{j}$ (subscript 1 denotes scheme 1), which is the $C_{l}$ on the airfoil lift curve corresponding to $\left(\alpha_{s}\right)_{j}$, as shown in Fig. 3.3. Similarly, $\left(C_{m t, 1}\right)_{j}$, the target $C_{m}$, is the $C_{m}$ on the airfoil $C_{m}-\alpha$ curve corresponding to $\left(\alpha_{s}\right)_{j}$. Hence, compute the residuals for scheme 1 as $\left(\Delta C_{l, 1}\right)_{j}=\left(C_{l s}\right)_{j}-\left(C_{l t, 1}\right)_{j}$ and $\left(\Delta C_{m, 1}\right)_{j}=\left(C_{m s}\right)_{j}-\left(C_{m t, 1}\right)_{j}$.

5. Perturb $\delta_{1}$ at section $j$ by adding a small perturbation $p$.

6. Compute the wing aerodynamic characteristics with the perturbed decambering using VLM3D; for example, the resulting $C_{l}$ and $C_{m}$ for section $j$ are denoted by $\left(C_{l_{p}}\right)_{j}$ and $\left(C_{m_{p}}\right)_{j}$. Hence, compute the $j^{t h}$ column of $J_{l 1}$ and $J_{m 1}$ using Eqns. 3.3 and 3.4.

7. Residuals for Scheme 2: Compute the local effective angle of attack of each section using the perturbed decambering; for example, the local effective angle of attack of section $j$ is obtained by setting $\left(C_{l s e c}\right)=\left(C_{l p}\right)_{j}$ in Eqn. 3.7. This effective angle of attack is denoted by $\left(\alpha_{p}\right)_{j}$. The line joining 
the points $\left[\left(\alpha_{s}\right)_{j},\left(C_{l_{s}}\right)_{j}\right]$ and $\left[\left(\alpha_{p}\right)_{j},\left(C_{l_{p}}\right)_{j}\right]$ is called the "trajectory line," as it determines the linearized trajectory of how a point on the $C_{l}-\alpha$ curve defined by the local section $\alpha_{e f f}$ and local section $C_{l}$ moves with changes in $\delta_{1}$. Therefore, in scheme 2 , the target $C_{l},\left(C_{l t, 2}\right)_{j}$, of section $j$ for example, is the point of intersection between the trajectory line for section $j$ and the airfoil lift curve, as illustrated in Fig. 3.3. The corresponding $\alpha$ is $\left(\alpha_{t, 2}\right)_{j}$ and $\left(C_{m t, 2}\right)_{j}$ is the target $C_{m}$ on the airfoil $C_{m}-\alpha$ curve corresponding to $\left(\alpha_{t, 2}\right)_{j}$.

The residuals for scheme 2 are now computed as $\left(\Delta C_{l, 2}\right)_{j}=\left(C_{l s}\right)_{j}-\left(C_{l t, 2}\right)_{j}$ and $\left(\Delta C_{m, 2}\right)_{j}=\left(C_{m s}\right)_{j}-\left(C_{m t, 1}\right)_{j}$.

8. Reset the value of $\delta_{1}$ at section $j$ to $\left(\delta_{1 s}\right)_{j}$.

9. Cycle through steps 5-6 for all values of the section index $j$ to compute all the columns of $J_{l 1}$ and $J_{m 1}$.

10. Repeat steps 5-9 now perturbing $\delta_{2}$ instead of $\delta_{1}$ to compute $J_{l 2}$ and $J_{m 2}$. In this process, the computation of the residuals for scheme 2 in step 7 is ignored, as they have already been computed.

11. Using the Newton equation in Eqn. 3.1, compute the correction vector $\delta \mathbf{x}$. Update the values of $\delta_{1 s}$ and $\delta_{2 s}$ by adding the correction vector $\delta \mathbf{x}$ multiplied by a user-specified damping factor $D$ (also referred to under-relaxation factor). In the current work, $D$ has been set to 0.1 .

This iteration process is carried out until all the residuals have converged to a specified tolerance. In the current work a tolerance of 0.001 has been used in all the examples.

\subsection{Multiple intersections in scheme 2}

Figure 3.4 illustrates an important consequence of using an inclined trajectory line for determining the target $C_{l}$ in scheme 2 . 
Three possible ways in which the trajectory line may intersect the airfoil lift curve are illustrated in Fig. 3.4: (i) the trajectory line marked as L1 intersects the airfoil lift curve at a single pre-stall point, (ii) the trajectory line marked as L2 intersects the airfoil lift curve at multiple points and (iii) the trajectory line marked as L3 intersects the airfoil lift curve at a single point in the post-stall region. While there is no ambiguity in determining the values of the target $C_{l}$ for lines L1 and L3, there are clearly three possible choices for the target $C_{l}$ for line L2. This illustration clearly demonstrates that it is possible to obtain multiple solutions for post-stall conditions; a fact, that was apparently first suggested by von Kármán (see Ref. 4) and has since been discussed by several researchers. ${ }^{3-6,8,9,12}$ However, the approach in scheme 2 is novel because this scheme is believed to be the first one in which the possibility of multiple solutions for high angles of attack is brought to light right during the iteration process. Earlier approaches including scheme 1 were able to identify the existence of multiple solutions only as a result of obtaining multiple converged solutions with different initial conditions in the iteration procedure.

The existence of multiple intersections also presents a dilemma in choosing an appropriate target $C_{l}$ from the possible multiple solutions. The following procedure was developed for making the choice during the intersection process. At each step of the iteration, each of the sections on all of the wings is examined to identify those with single intersections, as identified by points A and B in Fig. 3.4. The target $C_{l}$ values for these sections are identified without ambiguity. Using a logical switch called "lpoststall" in the code, each of these sections are also tagged as "stalled" or "unstalled" depending on whether the $\alpha$ for the intersection point is greater than or less than the $\alpha$ for $C_{l \max }$. The sections with multiple intersection points are then examined. Using the trajectory line L2 in Fig. 3.4 for example, the intersection point 1 is chosen if the logical switch lpoststall 
for the section is unstalled and the intersection point 3 is chosen if the logical switch for the section is stalled. This procedure is illustrated using a flow chart in Fig. 3.5.

Next, another logic is applied in which all the sections of the wings are scanned to identify sets of contiguous sections, all of which have multiple intersections and all of which are also tagged as unstalled. If any of these sets of contiguous sections are bound on both sides by sections tagged as stalled, then all the sections in this set are also tagged stalled. This logic removed any occurrence of unstalled regions with multiple-intersections sandwiched between two stalled regions.

The values of the logical switch for all the sections are carried over from one iteration to the next as well as from one $\alpha$ to the next when performing the analysis for a sequence of angles of attack. Thus, if a section gets tagged as "stalled" at any point in the iteration, then it remains tagged as "stalled" unless the section ends up with a trajectory line like L1 in Fig. 3.4. 


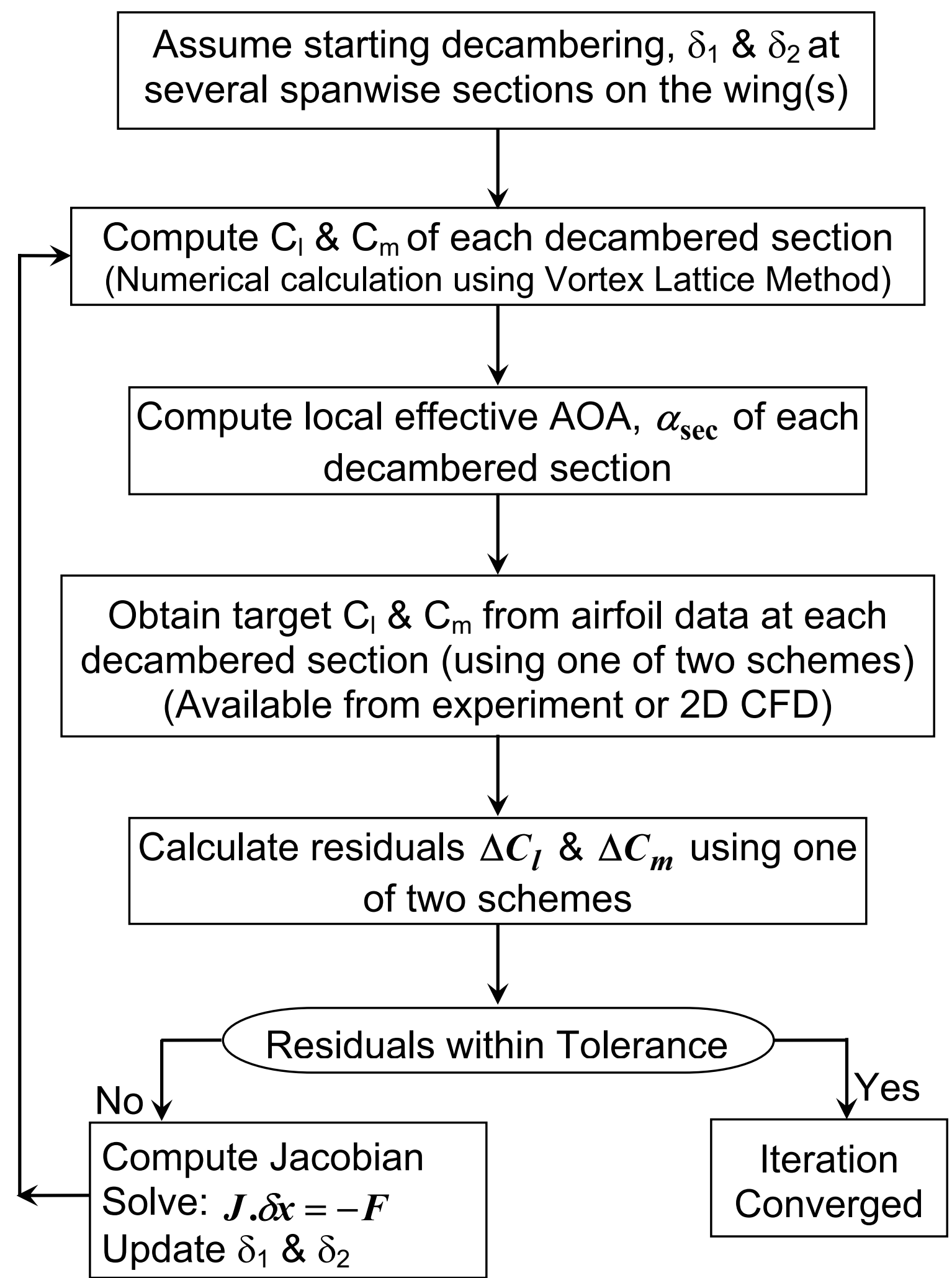

Figure 3.2: Flow chart of the iterative decambering approach for a wing(s). 


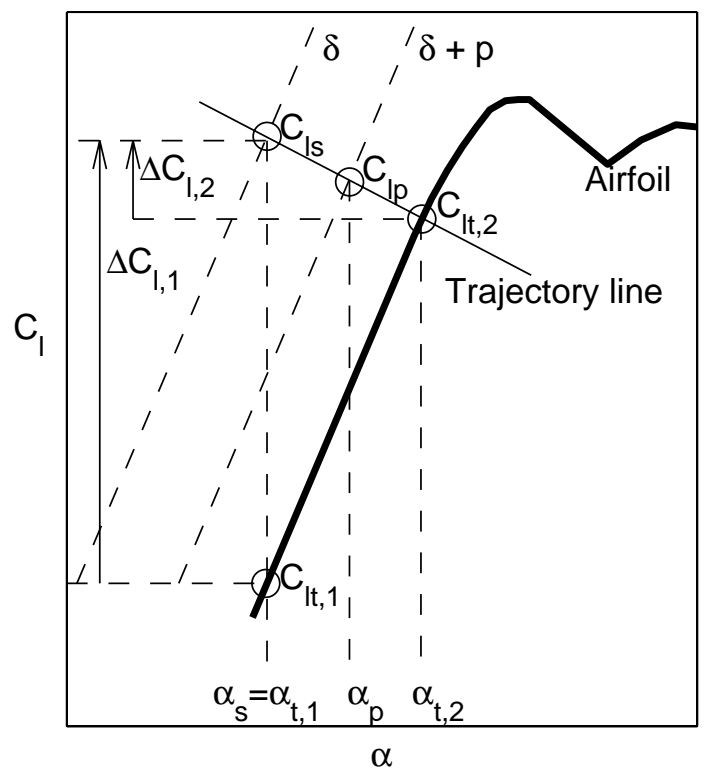

Figure 3.3: Illustration of the differences in the computation of the residuals using schemes 1 and 2 .

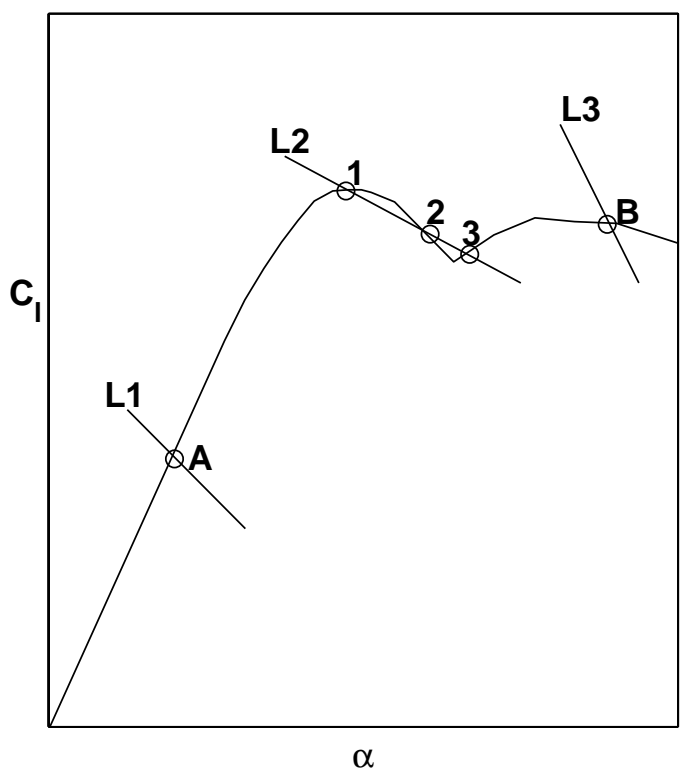

Figure 3.4: Illustration of the different ways in which a trajectory line may intersect the airfoil $C_{l}-\alpha$ curve. 


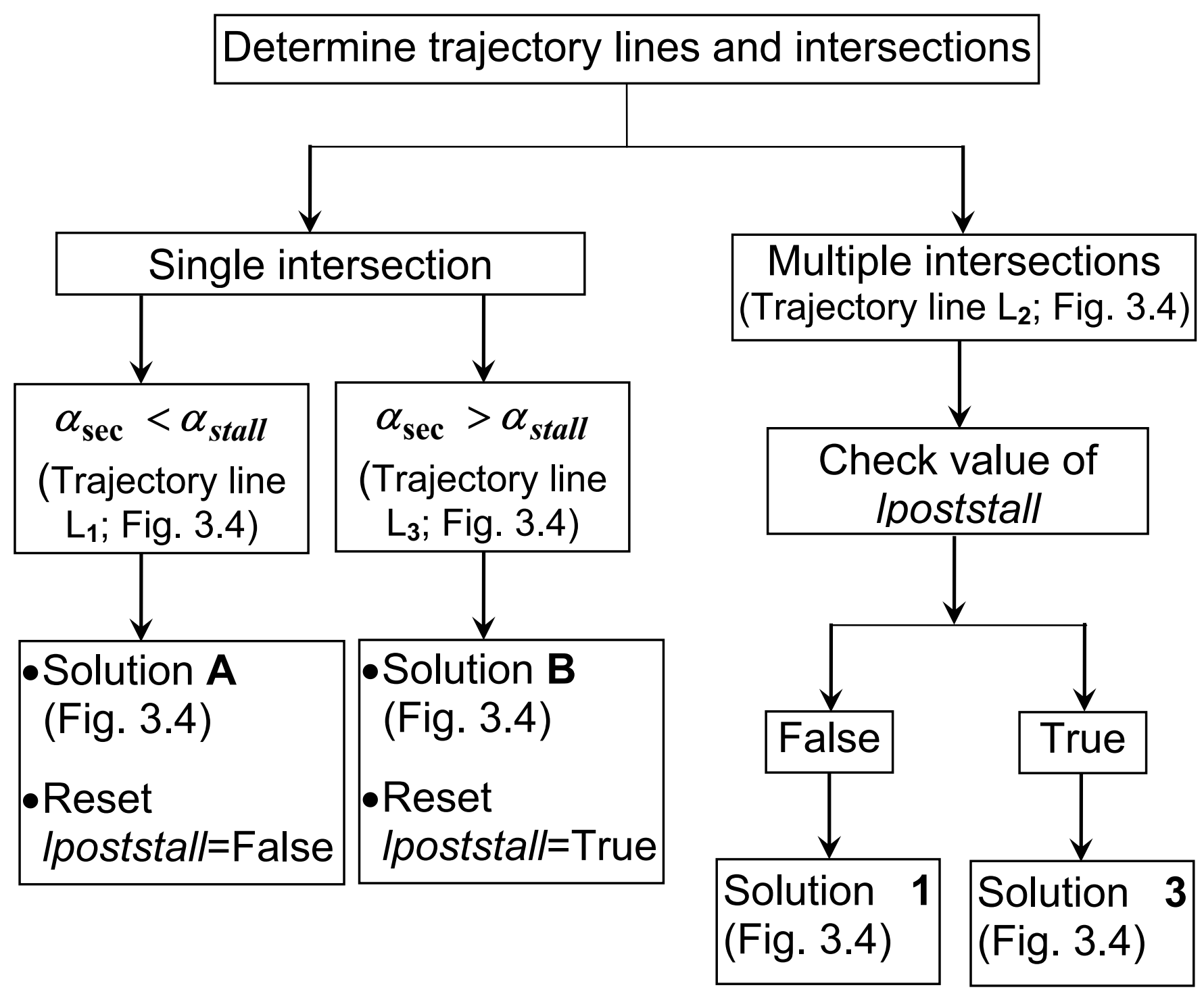

Figure 3.5: Flow chart for handling multiple intersections in Scheme 2 at a particular section on the wing(s). 


\section{Chapter 4}

\section{Results}

The iterative decambering approach discussed in Chapter 3 has been implemented for the analysis of multiple-lifting-surface configurations in VLM3D, a custom VLM code. In this chapter, post-stall results from VLM3D are presented for several airfoil lift curves, different planform shapes and several lifting-surface configurations. The computation of the residual has been implemented using two schemes and the effectiveness of the two schemes are compared. The examples in this chapter are presented in five sections as follows:

1. Section 4.1: In this section, the examples have been used to compare the predicted results from the current method with experimental results from Naik and Ostowari. ${ }^{17}$ In their work, ${ }^{17}$ experimental $C_{l}$ - $\alpha$ data for the NACA 4415 airfoil at Reynolds numbers of 0.5 million and 0.75 million are presented along with experimental $C_{L^{-}} \alpha$ data for finite constant-chord wings of several aspect ratios with the same airfoil and at the same Reynolds numbers. For the examples in this section, the two-dimensional experimental data has been used as input for the method and the predicted finite-wing lift curves have been compared with the experimental data. The examples have been used to compare the effectiveness of the two schemes and to illustrate how multiple converged solutions can result at post-stall angles of attack when different starting solutions are used for the iteration. 
2. Section 4.2: The objective of the second set of examples used in this section was to study the effect of planform shape on the stall characteristics of a wing. Tapered wings of different taper ratios are used to study where the wing first stalls and how the stall progresses along the span with increasing angle of attack.

3. Section 4.3: In this section, a wing-tail configuration is analyzed with the current method to demonstrate the capability of the current method to handle multiple-lifting-surface configurations. The effect of the wing stall on the aircraft pitching moment is shown to illustrate how the method can be used for providing information for the study of stability and control characteristics.

4. Section 4.4: A wing-canard configuration is used in this section to provide another example of an application to a multiple-lifting-surface configuration. This example illustrates how the canard stall behavior influences the wing lift distribution because of the downwash/upwash effects of the canard on the wing.

5. Section 4.5: In this section, an initial asymmetric distribution of $\delta_{1}$ is used for the iteration process to see if any asymmetries occur in the final converged $C_{l}$ distributions.

\subsection{Experimental Validation}

In this section, experimental two-dimensional data ${ }^{17}$ for a NACA 4415 airfoil at two Reynolds numbers of 0.5 million and 0.75 million, shown in Fig. 4.1, is used as input to generate post-stall results for rectangular wings of aspect ratios 12, 9, and 6 using VLM3D. The results from the iterative decambering approach are 


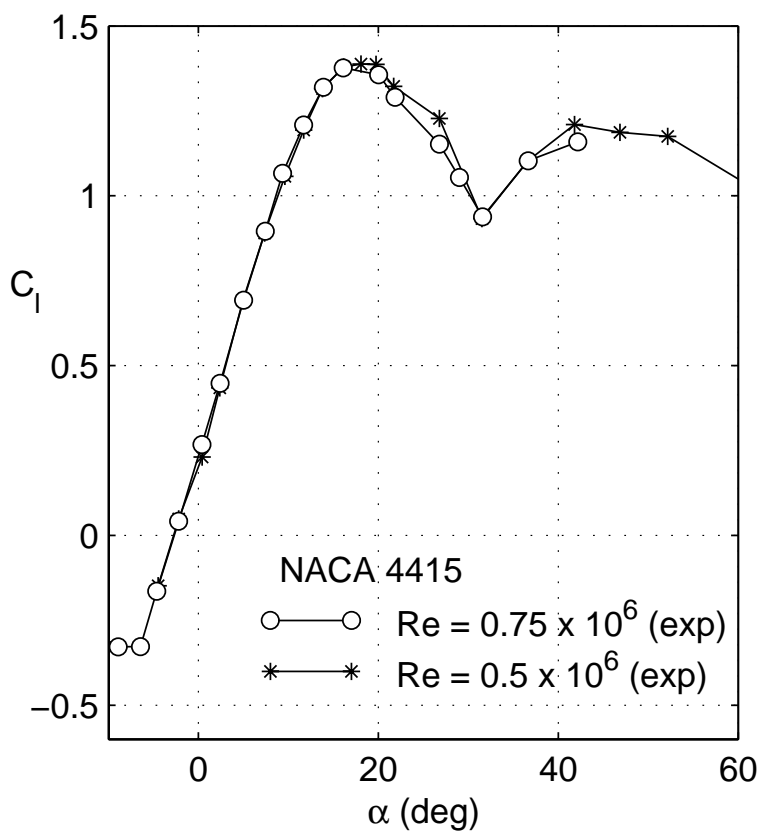

Figure 4.1: Airfoil lift curves for the NACA 4415 airfoil from Naik and Ostowari ${ }^{17}$.

then compared with the experimental $C_{L^{-}} \alpha$ curves for the corresponding wings from the experimental data of Naik and Ostowari. ${ }^{17}$ The following sub-sections present the results for each case:

\subsubsection{Rectangular Wing $(\mathrm{AR}=12)$ with the NACA 4415 Airfoil at Re of 0.5 Million}

In this case, the airfoil $C_{l^{-}} \alpha$ curve is from the experimental data ${ }^{17}$ for the NACA 4415 airfoil at $\operatorname{Re}=0.5 \times 10^{6}$ (Fig. 4.1). A rectangular wing of aspect ratio 12 as shown in Fig. 4.2(a) is considered. Figure 4.3 shows the wing $C_{L^{-}} \alpha$ curves from VLM3D using schemes 1 and 2. In the same figure, the airfoil $C_{l^{-}} \alpha$ curve and the wing $C_{L^{-}} \alpha$ curve from experiment ${ }^{17}$ are also shown for comparison. In both schemes, the starting values of $\delta_{1}$ and $\delta_{2}$ for each $\alpha$ were taken from the converged values for the previous $\alpha$. For the first $\alpha$ of the sequence, $\delta_{1}$ was set to -40 deg and $\delta_{2}$ was set to 0 deg for both schemes. 

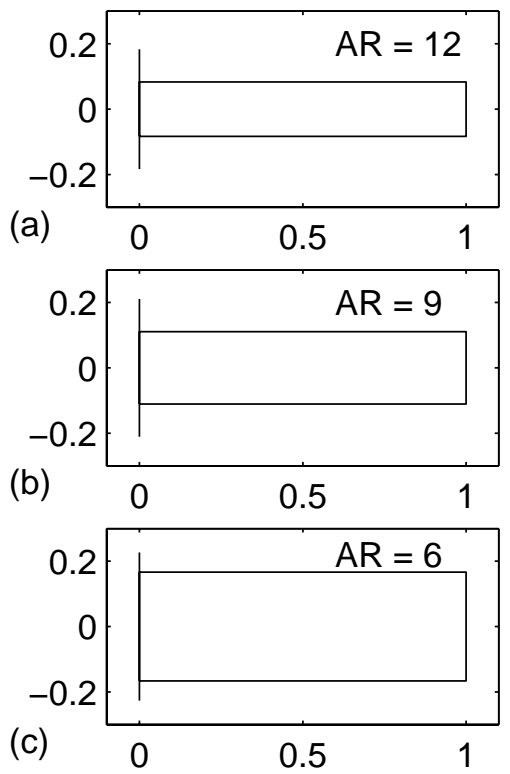

Figure 4.2: Planform of the rectangular wings (RHS shown) used in sec. 4.1.

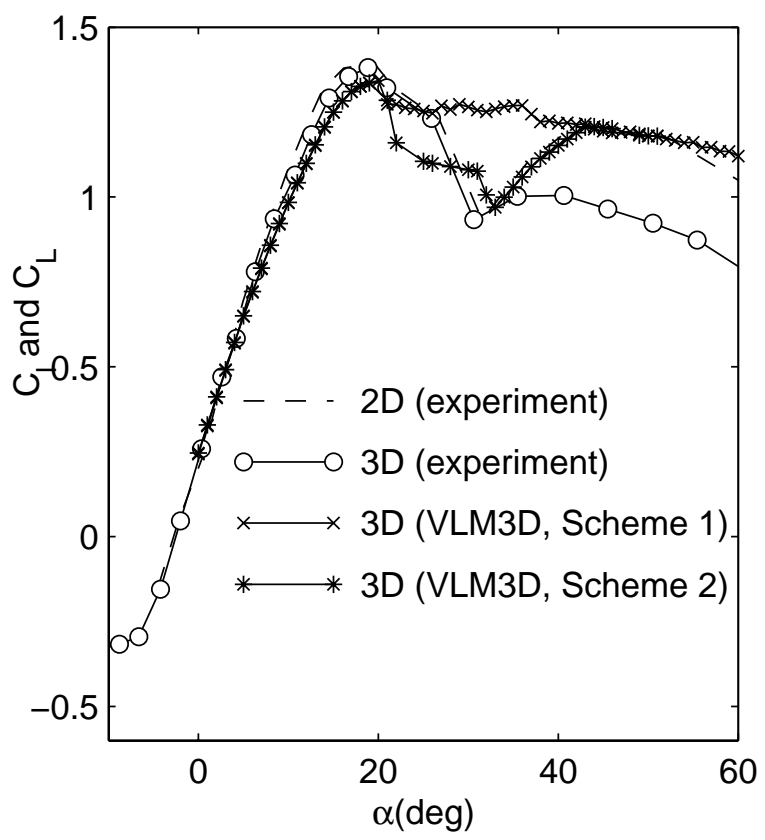

Figure 4.3: Wing $C_{L^{-}} \alpha$ predicted using schemes 1 and 2 for a rectangular wing of aspect ratio 12 , using a NACA 4415 airfoil at Reynolds number of 0.5 million. 

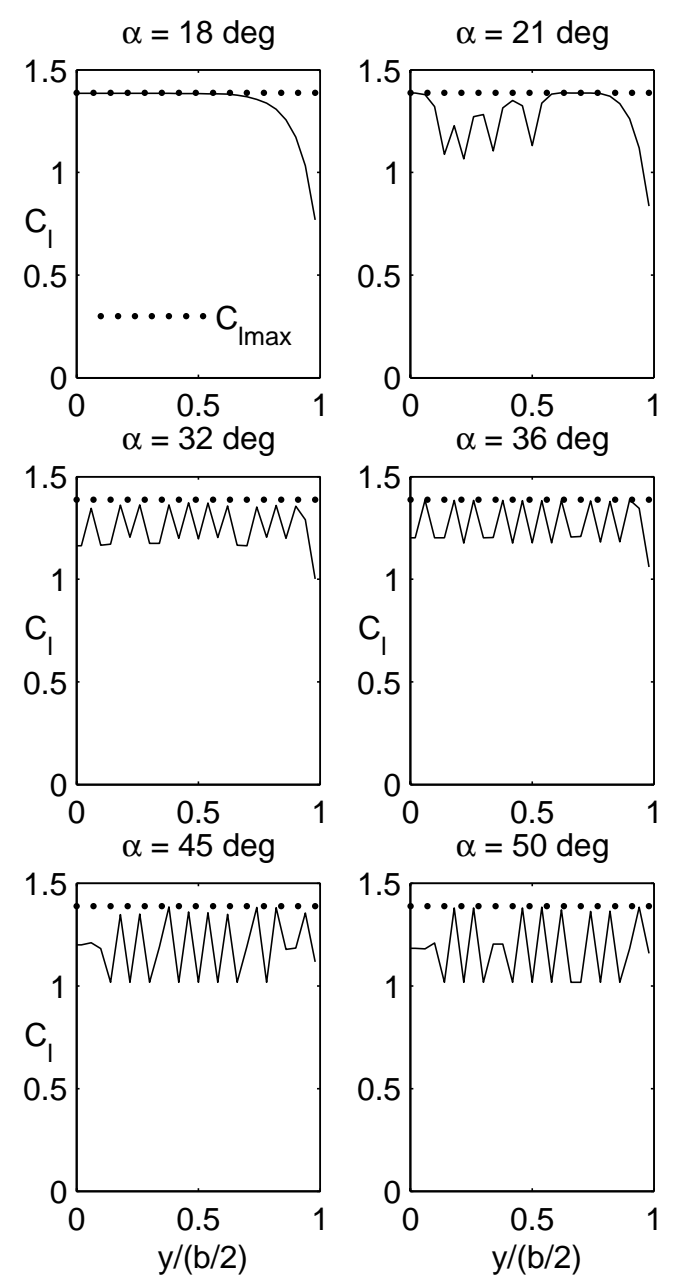

Figure 4.4: Spanwise $C_{l}$ distribution predicted for a rectangular wing of aspect ratio 12, using a NACA 4415 airfoil at Reynolds number of 0.5 million from scheme 1.

As is seen from Fig. 4.3, the two schemes result in identical predictions for the wing $C_{L}$ for pre-stall angles of attack. For post-stall conditions, the results of scheme 2 are closer to the experimental results. Figures 4.4 and 4.5 show the spanwise $C_{l}$ distributions for angles of attack 18, 21, 32, 36, 45 and 50 degrees from schemes 1 and 2 respectively. $C_{L \max }$ occurs at around $\alpha$ of 18 degrees. Experimental results for the spanwise $C_{l}$ distributions were not available for comparison. As seen from the results of scheme 1 in Fig. 4.4, there is substantial "sawtooth behavior" in the spanwise section $C_{l}$ distributions with regions of unstalled flow 

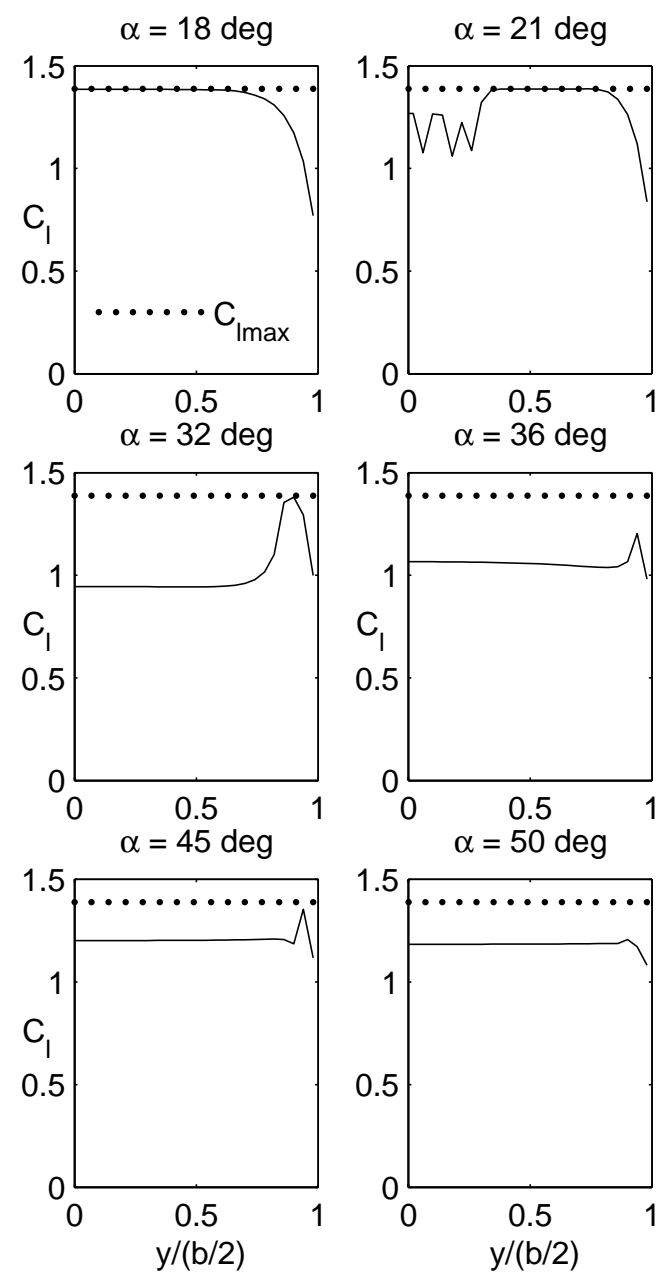

Figure 4.5: Spanwise $C_{l}$ distribution predicted for a rectangular wing of aspect ratio 12, using a NACA 4415 airfoil at Reynolds number of 0.5 million from scheme 2.

sandwiched between regions of stalled flow. Similar sawtooth oscillations have been reported by other researchers ${ }^{3-6,8,9,12}$ for post-stall angles of attack. Although these sawtooth results correspond to numerically converged solutions, it is believed that a real flow cannot have such spanwise oscillations. The results from scheme 2, shown in Fig. 4.5, do not exhibit these "sawtooth oscillations" for a majority of the conditions. For this reason, scheme 2 is considered more acceptable. The results from both the schemes, however, have numerically converged to within a tolerance of 0.001 in $\Delta C_{l}$ and $\Delta C_{m}$. 
The wing $C_{L}$ from scheme 2 in Fig. 4.3 shows that as the $\alpha$ is increased to $18 \mathrm{deg}$, the $C_{L}$ continues to increase. At this condition the entire wing remains unstalled as the local section $C_{l}$ values are less than the $C_{L \max }$ of 1.39 . This can be confirmed by examining the spanwise section $C_{l}$ distribution from scheme 2 in Fig. 4.5 for $\alpha$ of $18 \mathrm{deg}$. At $18 \mathrm{deg}$ the inboard portion of the wing is close to stall. As the $\alpha$ is increased between $18 \mathrm{deg}$ and $21 \mathrm{deg}$, a part of the inboard portion of the wing stalls and another part is close to stall as can be seen from the spanwise section $C_{l}$ distribution for $\alpha$ of $21 \mathrm{deg}$ in Fig. 4.5. As the $\alpha$ is increased beyond 21 deg, the spanwise extent of the stalled portion increases. At 32 deg most of the wing has stalled and the outboard portion is close to stall as shown in Fig. 4.5. Between $32 \mathrm{deg}$ and $44 \mathrm{deg}$ the $C_{l}$ on the stalled portion of the wing increases. The wing $C_{L}$ therefore increases between $32 \mathrm{deg}$ and $44 \mathrm{deg}$ as shown in Fig. 4.3. Beyond $44 \mathrm{deg}$, the $C_{l}$ on the wing does not increase anymore. In fact, it remains almost constant at 1.2, as shown in Fig. 4.5 for $\alpha$ of 45 deg. At $50 \mathrm{deg}$, the entire wing has stalled as seen from the spanwise section $C_{l}$ distribution for $\alpha$ of $50 \mathrm{deg}$. Therefore the wing $C_{L}$ decreases between $44 \mathrm{deg}$ and $51 \mathrm{deg}$.

The wing $C_{L^{-}} \alpha$ from scheme 1 on the other hand does not show a marked decrease when the $\alpha$ is increased between 18 and 32 deg as shown in Fig. 4.3. This can be explained by investigating the spanwise section $C_{l}$ distributions from scheme 1 for $\alpha$ of 32, 36, 45 and 50 deg in Fig. 4.4. There is considerable sawtooth behavior and no large-scale stalling of the wing. Even at 50 deg there are several unstalled sections sandwiched between stalled sections. Hence, although the wing $C_{L}$ drops after 18 deg as some sections stall, it does not show any marked decrease or a $C_{L}$ minimum.

It is to be mentioned that a sequence of angles of attack from -5 to $60 \mathrm{deg}$ was analyzed using scheme 2 and some angles of attack did not converge. In Fig. 4.3 the wing $C_{L}$ for only the converged angles of attack are plotted for scheme 2. For 


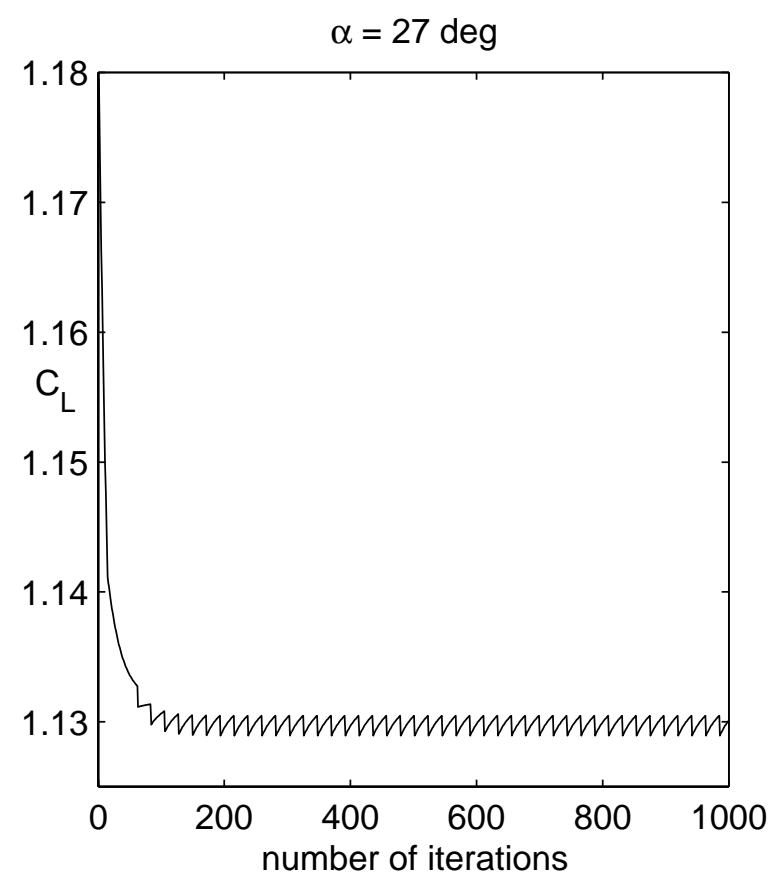

Figure 4.6: Wing $C_{L}$ variation with number of iterations for a rectangular wing of aspect ratio 12, using a NACA 4415 airfoil at Reynolds number of 0.5 million from scheme 2 .

example, the iterations did not converge at $\alpha$ of $27 \mathrm{deg}$ and Fig. 4.6 shows the variation of the wing $C_{L}$ with number of iterations for $\alpha$ of $27 \mathrm{deg}$. It is seen that the convergence plot for the wing $C_{L}$ exhibits an undamped periodic behavior and oscillates within a certain range and does not converge to any particular value. A probable explanation for this behavior is the existence of multiple solutions (a maximum of 5 possible solutions for the airfoil data considered) at $27 \mathrm{deg}$. Scheme 2 uses an inclined trajectory line in order to determine a solution wherein, whenever more than two solutions are possible the intermediate solutions are neglected and either the maximum or minimum solution is chosen depending upon "lpoststall". In the particular case of $27 \mathrm{deg}$, the maximum or minimum solution is not within the required tolerance and so neither of them is chosen. And any other possible solution is an intermediate solution and therefore that solution is also not chosen. As a result the iteration oscillates between the maximum and 
minimum values but does not converge.

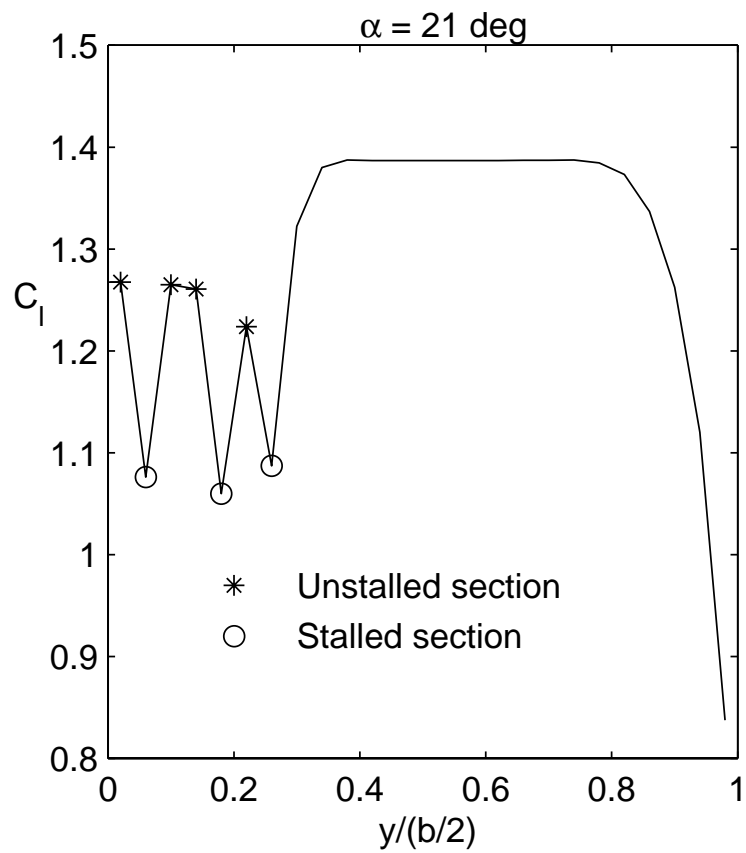

Figure 4.7: Sawtooth in spanwise $C_{l}$ distribution for a rectangular wing of aspect ratio 12, using a NACA 4415 airfoil at Reynolds number of 0.5 million from scheme 2.

Also, for $\alpha$ of $21 \mathrm{deg}$, oscillations were observed in the spanwise section $C_{l}$ distribution as shown in Fig. 4.5. This sawtooth region at $\alpha$ of $21 \mathrm{deg}$ is examined closely in Fig. 4.7 and it illustrates that the sawtooth occurs because of unstalled sections sandwiched between stalled sections. Such oscillations were observed for some angles of attack in the sequence, some of which have final converged solutions. Figure 4.8 shows the section $C_{l}$ plotted against the section effective angle of attack on the airfoil $C_{l^{-}} \alpha$ curve for the points corresponding to the upper and lower corners of the sawtooth region. It is seen that the upper corners of the sawtooth region (marked by "**" in Figs. 4.7 and 4.8) have converged to the unstalled region of the airfoil $C_{l^{-}} \alpha$ curve as shown in Fig. 4.8 and the lower corners (marked by "o" in Figs. 4.7 and 4.8) have converged to the stalled region of the airfoil $C_{l^{-}} \alpha$ curve as shown in Fig. 4.8. Because of the fact that the trajectory 


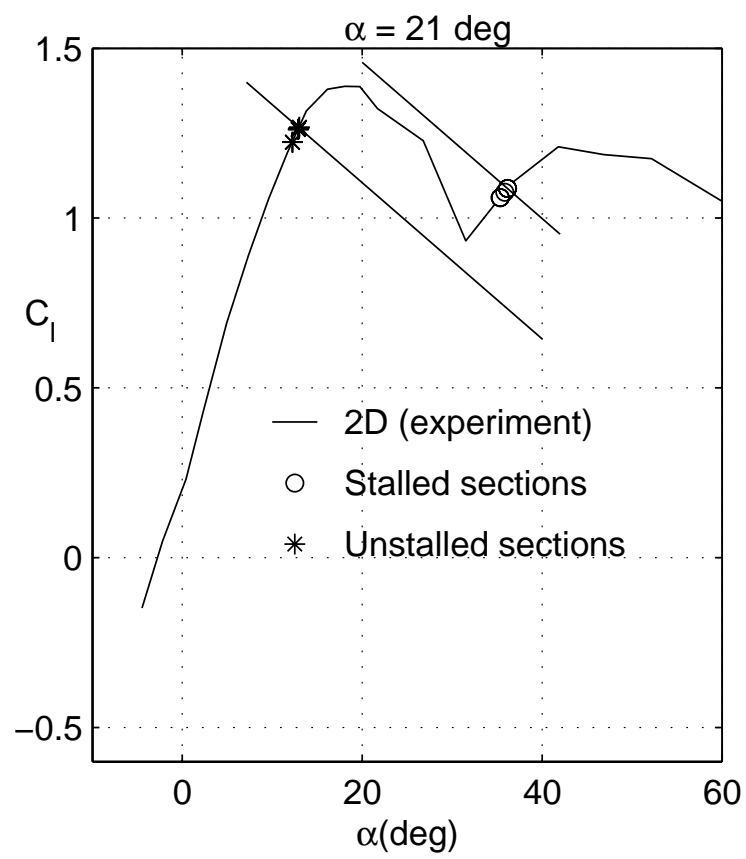

Figure 4.8: Location of the upper and lower corners of the sawtooth region shown in Fig. 4.7 on the NACA 4415 airfoil $C_{l^{-}} \alpha$ curve.

lines for the upper corner points intersect the airfoil $C_{l^{-}} \alpha$ curve at only a single point, the post-stall logic was unable to remove such oscillations.

\subsubsection{Effect of initial conditions on the iterations for Scheme 2}

It must be mentioned that for a majority of the cases, Scheme 2 is successful in converging to realistic solutions with no "sawtooth behavior". It is only for a few conditions that the converged solution has a sawtooth behavior (e.g. $\alpha=21 \mathrm{deg}$ in Fig. 4.5) or the solution does not converge due to an undamped periodic convergence pattern as shown in Fig. 4.6. Figure 4.9 shows the wing $C_{L^{-}} \alpha$ predicted using scheme 2 for two different starting conditions for the Newton Iteration: (a) $\delta_{1}=-40 \mathrm{deg}$ for all sections at each $\alpha$ and (b) $\delta_{1}=0$ deg for all sections at each $\alpha$. Figure 4.10 shows the spanwise $C_{l}$ distributions for the two cases for $\alpha$ of 18 


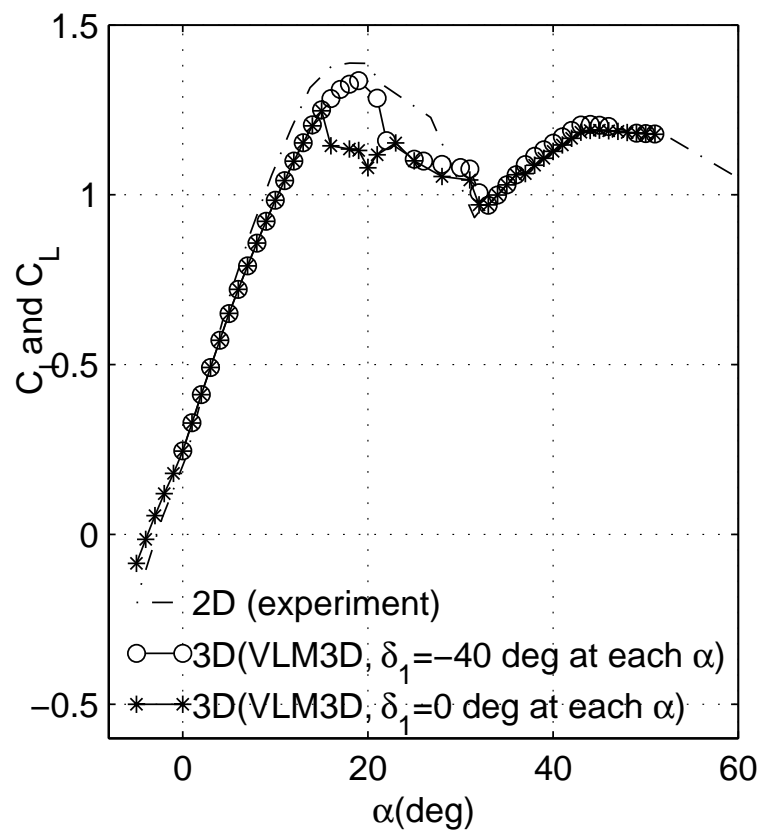

Figure 4.9: Wing $C_{L^{-}} \alpha$ predicted for a rectangular wing of aspect ratio 12 using a NACA 4415 airfoil at Reynolds number of 0.5 million for different starting values of $\delta_{1}$.

deg. The results clearly illustrate that multiple solutions are possible for poststall conditions. Furthermore, the results do not provide any clear guidelines as to which is the correct solution. It can however be said that the different schemes and starting assumptions predict the wing $C_{L}$ for a given post-stall angle of attack within a small scatter band. The scatter shown confirms the possibility of multiple solutions at post-stall angles of attack pointed out by other researchers ${ }^{3-6,8,9,12}$ and the sensitivity of post-stall solutions to initial conditions as well as schemes used for the Newton iteration.

\subsubsection{Rectangular Wing $(\mathrm{AR}=9)$ with the NACA 4415 Airfoil at Re of 0.5 Million}

For this case also, the airfoil $C_{l^{-}} \alpha$ curve is from experiment and is as shown in Fig. 4.1 for $\operatorname{Re}=0.5 \times 10^{6}$. A rectangular wing of aspect ratio 9 , as shown in 

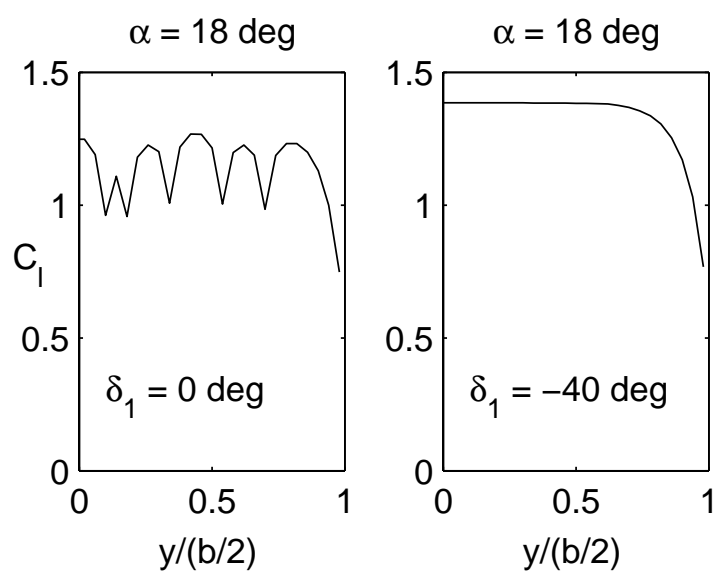

Figure 4.10: Spanwise section $C_{l}$ predicted for a rectangular wing of aspect ratio 12 using a NACA 4415 airfoil at Reynolds number of 0.5 million for different starting values of $\delta_{1}$.

Fig. 4.2, is studied. Figure 4.11 shows the wing $C_{L^{-}} \alpha$ curves from VLM3D using schemes 1 and 2. In the same figure the airfoil $C_{l^{-}} \alpha$ curve and the wing $C_{L^{-}} \alpha$ curve from experiment ${ }^{17}$ are also shown for comparison. In both schemes, the starting values of $\delta_{1}$ and $\delta_{2}$ were taken from the converged results of the previous $\alpha$. For the first $\alpha$ of the sequence, $\delta_{1}$ was set to $-40 \mathrm{deg}$ and $\delta_{2}$ was set to $0 \mathrm{deg}$ for both schemes. A sequence of angles of attack from -5 to $60 \mathrm{deg}$ was used and a few angles of attack did not converge for scheme 2. In Fig. 4.11 the wing $C_{L}$ for only the converged angles of attack are plotted for scheme 2 .

It is evident again from Fig. 4.11 that in comparing the results of the two schemes with the experimental data, scheme 2 gives a better comparison with experiment. Figs. 4.12 and 4.13 show the spanwise section $C_{l}$ distribution for $\alpha$ of $20,21,32,42,44$ and 50 degrees from scheme 1 and scheme 2 respectively. $C_{\text {Lmax }}$ occurs at around $\alpha$ of 20 degrees. Experimental results for the spanwise $C_{l}$ distributions were not available for comparison. As seen from the results of scheme 1 in Fig. 4.12, there is substantial sawtooth behavior in the spanwise section $C_{l}$ distributions. This sawtooth behavior is not present in the results of scheme 2 shown in Fig. 4.13. Results from both schemes have numerically converged to 


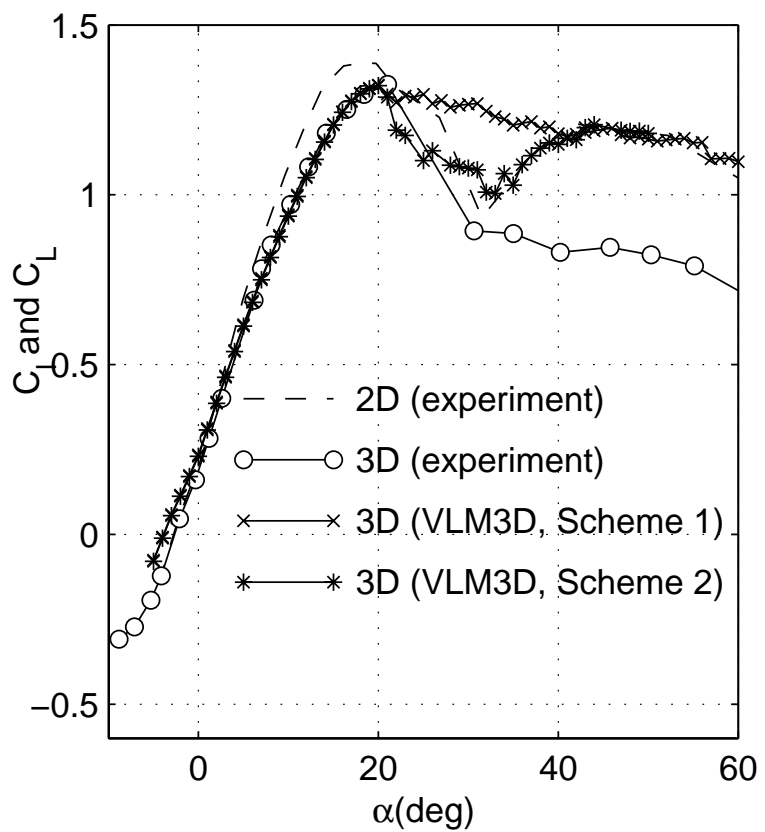

Figure 4.11: Wing $C_{L^{-}} \alpha$ predicted using schemes 1 and 2 for a rectangular wing of aspect ratio 9, using a NACA 4415 airfoil at Reynolds number of 0.5 million.

within a tolerance of 0.001 in $\Delta C_{l}$ and $\Delta C_{m}$.

\subsubsection{Rectangular Wing $(\mathrm{AR}=6)$ with the NACA 4415 Airfoil at Re of 0.5 Million}

In this case also, the airfoil $C_{l^{-}} \alpha$ curve is from experiment and is as shown in Fig. 4.1 for $\operatorname{Re}=0.5 \times 10^{6}$. A rectangular wing of aspect ratio 6 , as shown in Fig. 4.2, is studied. Figure 4.14 shows the wing $C_{L^{-}} \alpha$ curve from VLM3D using scheme 2. In the same figure the airfoil $C_{l^{-}} \alpha$ curve and the wing $C_{L^{-}} \alpha$ curve from experiment ${ }^{17}$ are also shown for comparison. For this case, scheme 1 failed to converge for post-stall angles of attack. As in the previous cases, the starting values of $\delta_{1}$ and $\delta_{2}$ were taken from the converged results of the previous $\alpha$. For the first $\alpha$ of the sequence, $\delta_{1}$ was set to $-40 \operatorname{deg}$ and $\delta_{2}$ was set to 0 deg. A sequence of angles of attack from -5 to 60 deg was used and a few angles of attack did not converge for scheme 2. In Fig. 4.14 the wing $C_{L}$ of only the converged angles of 

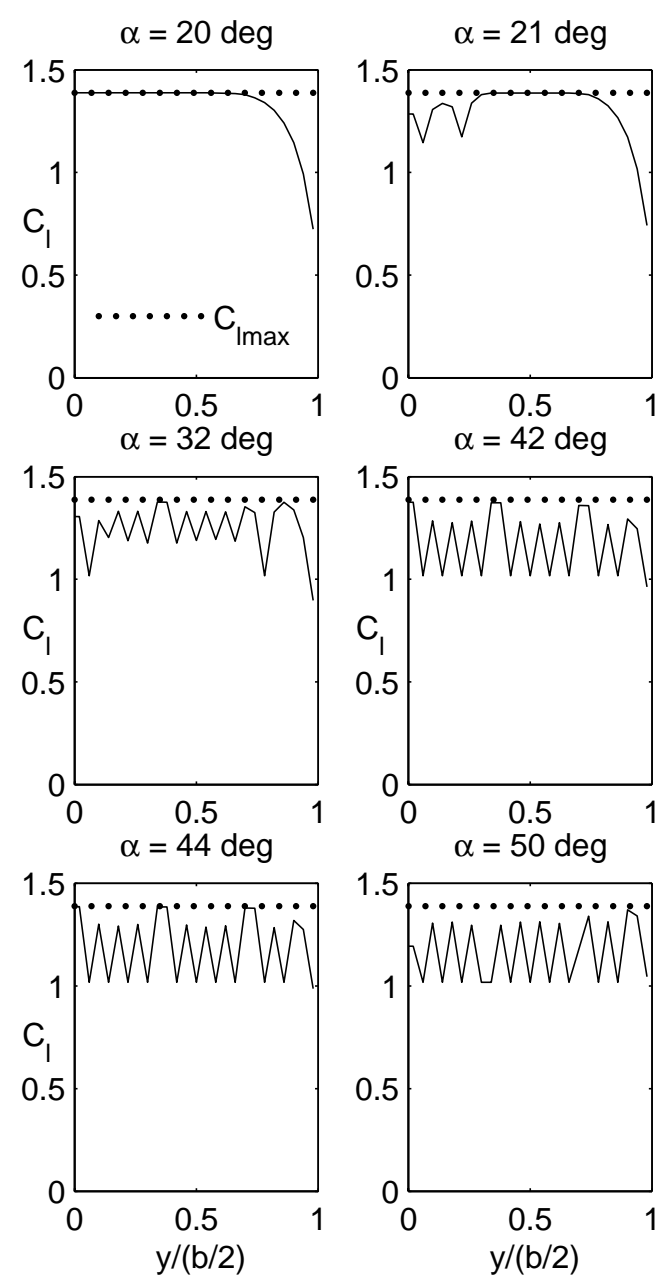

Figure 4.12: Spanwise $C_{l}$ distribution predicted for a rectangular wing of aspect ratio 9, using a NACA 4415 airfoil at Reynolds number of 0.5 million from scheme 1.

attack are plotted for scheme 2 .

Fig. 4.15 shows the spanwise section $C_{l}$ distribution for $\alpha$ of $21,22,32$, and 43 degrees from scheme 2. The trends are similar to those seen in sec. 4.1.1 for aspect ratio of 12 . 

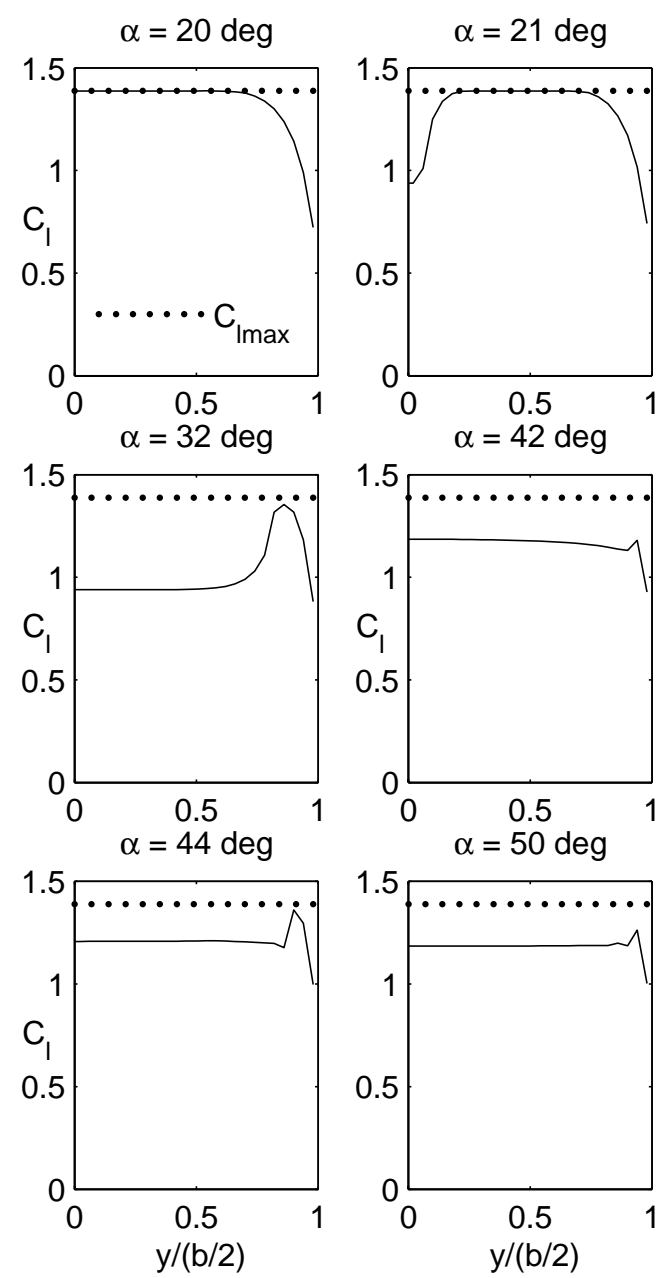

Figure 4.13: Spanwise $C_{l}$ distribution predicted for a rectangular wing of aspect ratio 9, using a NACA 4415 airfoil at Reynolds number of 0.5 million from scheme 2 .

\subsubsection{Rectangular Wing $(\mathrm{AR}=12)$ with the NACA 4415 Airfoil at Re of 0.75 Million}

In this case, the airfoil $C_{l^{-}} \alpha$ curve is from experiment and is as shown in Fig. 4.1 for $\operatorname{Re}=0.75 \times 10^{6}$. A rectangular wing of aspect ratio 12, as shown in Fig. 4.2, is studied. Figure 4.16 shows the wing $C_{L^{-}} \alpha$ curves from VLM3D using schemes 1 and 2. In the same figure the airfoil $C_{l^{-}} \alpha$ curve and the wing $C_{L^{-}} \alpha$ curve from experiment ${ }^{17}$ are also shown for comparison. In both schemes, the starting values of $\delta_{1}$ and $\delta_{2}$ were taken from the converged results of the previous $\alpha$. For the 


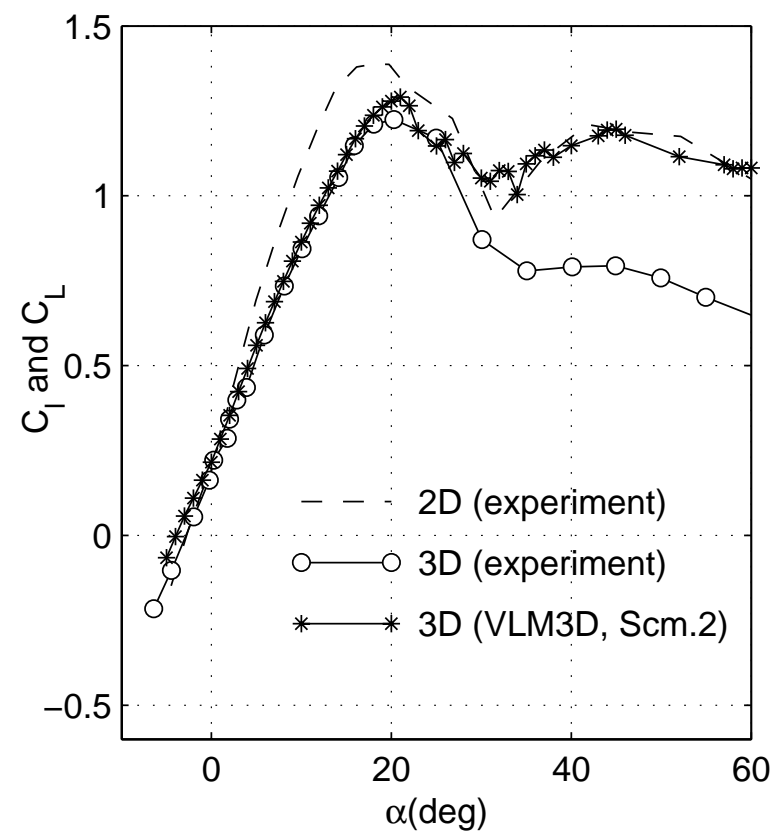

Figure 4.14: Wing $C_{L^{-}} \alpha$ predicted using scheme 2 for a rectangular wing of aspect ratio 6, using a NACA 4415 airfoil at Reynolds number of 0.5 million.

first $\alpha$ of the sequence, $\delta_{1}$ was set to $-40 \mathrm{deg}$ and $\delta_{2}$ was set to 0 deg for both schemes. A sequence of angles of attack from -5 to 60 deg was used and a few angles of attack did not converge for scheme 2. In Fig. 4.16, the wing $C_{L}$ of only the converged angles of attack are plotted for scheme 2 .

As observed before, it can be seen from Fig. 4.16 that scheme 2 gives a better comparison with experiment. Figs. 4.17 and 4.18 show the spanwise section $C_{l}$ distribution for $\alpha$ of 18, 21, 33, 36, 46 and 50 degrees from scheme 1 and scheme 2 respectively. $C_{L \max }$ occurs at around $\alpha$ of 18 degrees. Experimental results for the spanwise $C_{l}$ distributions were not available for comparison. As seen from the results of scheme 1 in Fig. 4.17, there is substantial sawtooth behavior in the spanwise section $C_{l}$ distributions. On the other hand, the results from scheme 2 for all conditions, except for $\alpha$ of 21 , deg do not exhibit the sawtooth oscillations. Results from both schemes have numerically converged to within a tolerance of 0.001 in $\Delta C_{l}$ and $\Delta C_{m}$. In general, the trends are similar to those seen in sec. 4.1.1 

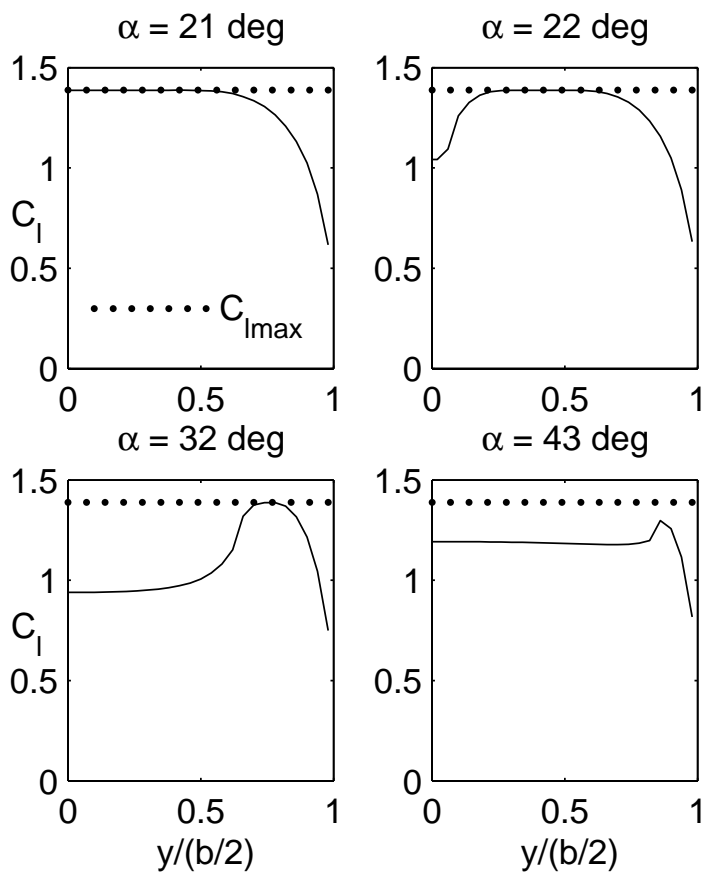

Figure 4.15: Spanwise $C_{l}$ distribution predicted for a rectangular wing of aspect ratio 6, using a NACA 4415 airfoil at Reynolds number of 0.5 million from scheme 2 .

for aspect ratio of 12 .

\subsubsection{Rectangular Wing $(\mathrm{AR}=9)$ with the NACA 4415 Airfoil at Re of 0.75 Million}

In this case the airfoil $C_{l^{-}} \alpha$ curve is from experiment and is as shown in Fig. 4.1 for $\operatorname{Re}=0.75 \times 10^{6}$. A rectangular wing of aspect ratio 9 as shown in Fig. 4.2 is studied. Figure 4.19 shows the wing $C_{L^{-}} \alpha$ curves from VLM3D using schemes 1 and 2. In the same figure the airfoil $C_{l^{-}} \alpha$ curve and the wing $C_{L^{-}} \alpha$ curve from experiment ${ }^{17}$ are also shown for comparison. In both schemes, the starting values of $\delta_{1}$ and $\delta_{2}$ were taken from the converged results of the previous $\alpha$. For the first $\alpha$ of the sequence, $\delta_{1}$ was set to $-40 \mathrm{deg}$ and $\delta_{2}$ was set to 0 deg for both schemes. A sequence of angles of attack from -5 to 60 deg was used and some angles of attack did not converge for scheme 2. In Fig. 4.19 the wing $C_{L}$ of only 


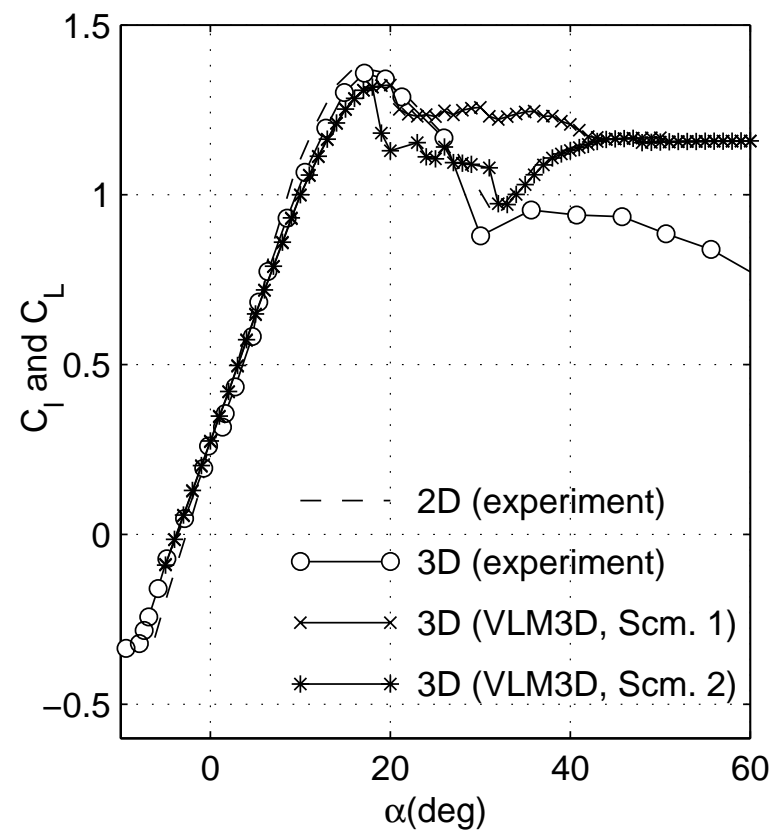

Figure 4.16: Wing $C_{L^{-}} \alpha$ predicted using schemes 1 and 2 for a rectangular wing of aspect ratio 12, using a NACA 4415 airfoil at Reynolds number of 0.75 million.

the converged angles of attack are plotted for scheme 2 .

As observed in earlier examples, it can be seen from Fig. 4.19 that scheme 2 gives a better comparison with experiment. Figs. 4.20 and 4.21 show the spanwise section $C_{l}$ distribution for $\alpha$ of 18,20,32,37, 47 and 50 degrees from scheme 1 and scheme 2 respectively. $C_{L \max }$ occurs at around $\alpha$ of 18 degrees. Experimental results for the spanwise $C_{l}$ distributions were not available for comparison. As seen from the results of scheme 1 in Fig. 4.20, there is substantial sawtooth behavior in the spanwise section $C_{l}$ distributions. Except for $\alpha$ of 20 deg, no sawtooth behavior is seen in the results for scheme 2. Results from both schemes have numerically converged to within a tolerance of 0.001 in $\Delta C_{l}$ and $\Delta C_{m}$. The trends are similar to those observed for earlier cases. 

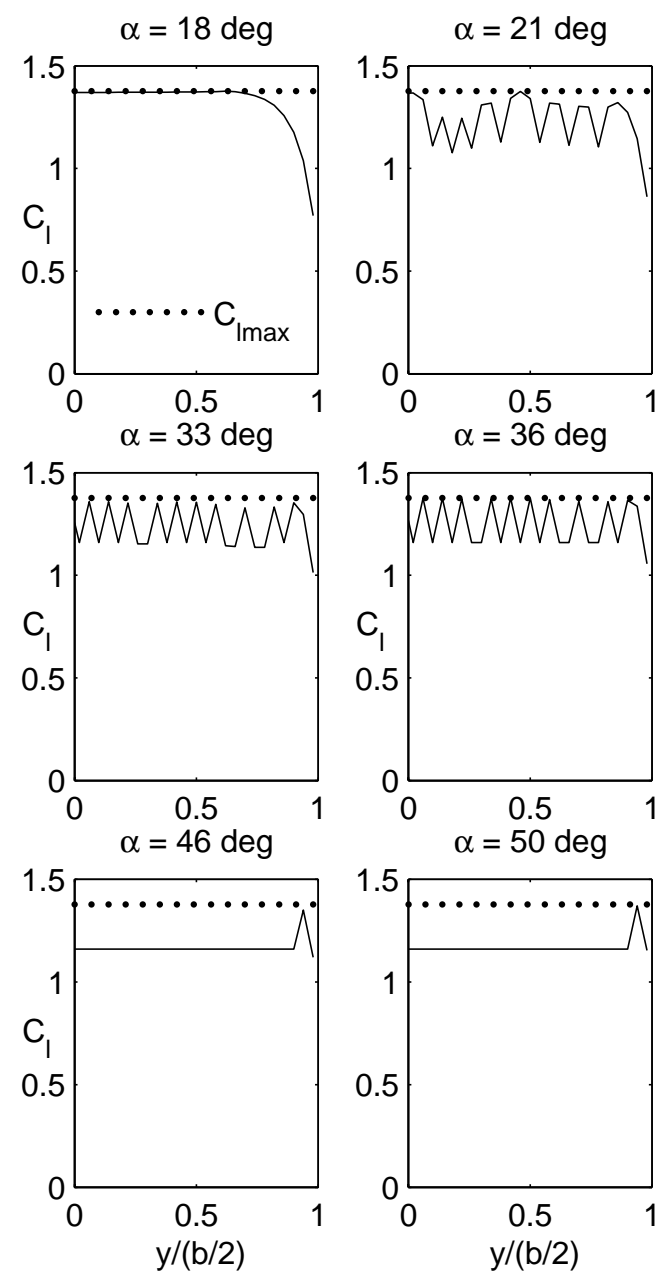

Figure 4.17: Spanwise $C_{l}$ distribution predicted for a rectangular wing of aspect ratio 12, using a NACA 4415 airfoil at Reynolds number of 0.75 million from scheme 1.

\subsubsection{Rectangular Wing $(\mathrm{AR}=6)$ with the NACA 4415 Airfoil at Re of 0.75 Million}

In this case the airfoil $C_{l^{-}} \alpha$ curve is from experiment and is as shown in Fig. 4.1 for $\operatorname{Re}=0.5 \times 10^{6}$. A rectangular wing of aspect ratio 6 as shown in Fig. 4.2 is studied. Figure 4.22 shows the wing $C_{L^{-}} \alpha$ curve from VLM3D using scheme 2 . In the same figure the airfoil $C_{l^{-}} \alpha$ curve and the wing $C_{L^{-}} \alpha$ curve from experiment ${ }^{17}$ are also shown for comparison. For this case scheme 1 failed to converge. As in the previous cases, the starting values of $\delta_{1}$ and $\delta_{2}$ were taken from the converged 

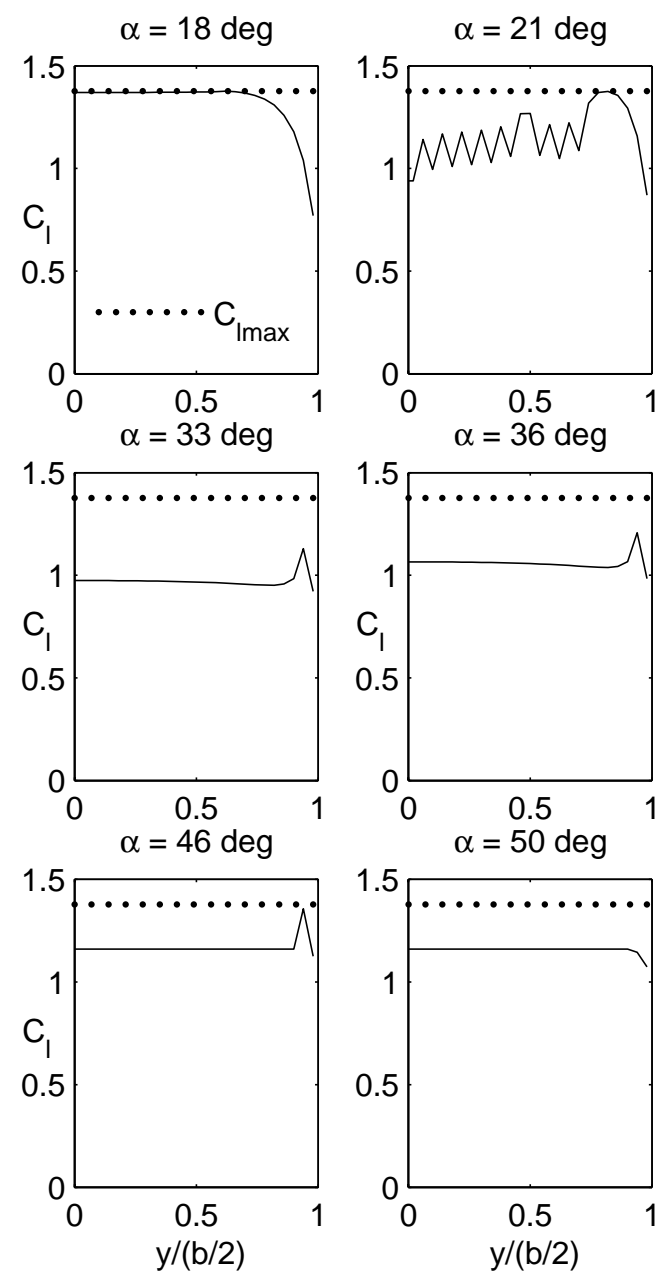

Figure 4.18: Spanwise $C_{l}$ distribution predicted for a rectangular wing of aspect ratio 12, using a NACA 4415 airfoil at Reynolds number of 0.75 million from scheme 2 .

results of the previous $\alpha$. For the first $\alpha$ of the sequence, $\delta_{1}$ was set to $-40 \mathrm{deg}$ and $\delta_{2}$ was set to 0 deg. A sequence of angles of attack from -5 to 60 deg was used and a few angles of attack did not converge for scheme 2. In Fig. 4.22 the wing $C_{L}$ of only the converged angles of attack are plotted for scheme 2 .

Fig. 4.23 shows the spanwise section $C_{l}$ distribution for $\alpha$ of $18,19,37$, and 60 degrees from scheme 2. $C_{L \max }$ occurs at around $\alpha$ of 18 degrees. Experimental results for the spanwise $C_{l}$ distributions were not available for comparison. Results from scheme 2 have numerically converged to within a tolerance of 0.001 in $\Delta C_{l}$ 


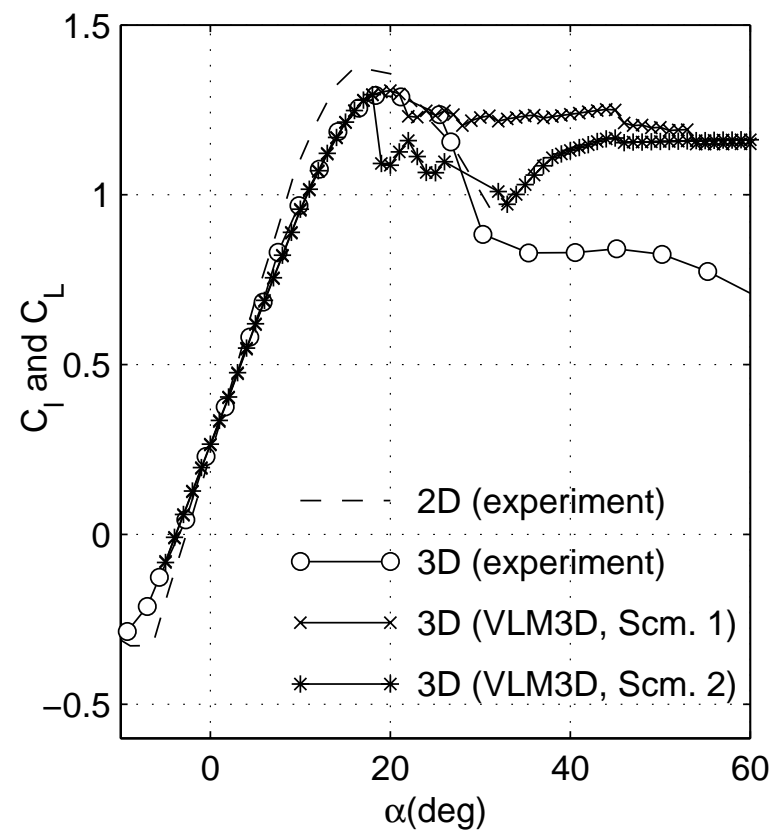

Figure 4.19: Wing $C_{L^{-}} \alpha$ predicted using schemes 1 and 2 for a rectangular wing of aspect ratio 9, using a NACA 4415 airfoil at Reynolds number of 0.75 million.

and $\Delta C_{m}$. Except for $\alpha$ of $19 \mathrm{deg}$, no sawtooth behavior is exhibited in the results of scheme 2 in Fig. 4.23.

\subsubsection{Changes to the Lift Curve with Change to Aspect Ratio}

In this subsection, the change to the wing $C_{L}$ with aspect ratio is studied. The airfoil $C_{l}-\alpha$ curve is from experiment and is as shown in Fig. 4.1 for Re of 0.5 million. The results from earlier subsections for the three rectangular wings of aspect ratios of 12, 9, and 6 as shown in Fig. 4.2 have been used. Figure 4.24 shows the $C_{L^{-}} \alpha$ curves of the three wings from VLM3D using scheme 2. In the same figure the airfoil $C_{l^{-}} \alpha$ curve from experiment ${ }^{17}$ is also shown for comparison. Figure 4.25 shows the results for the three wings from the experimental data of Naik and Ostowari. ${ }^{17}$ It is seen that the current approach is successful in capturing all of the important trends. 

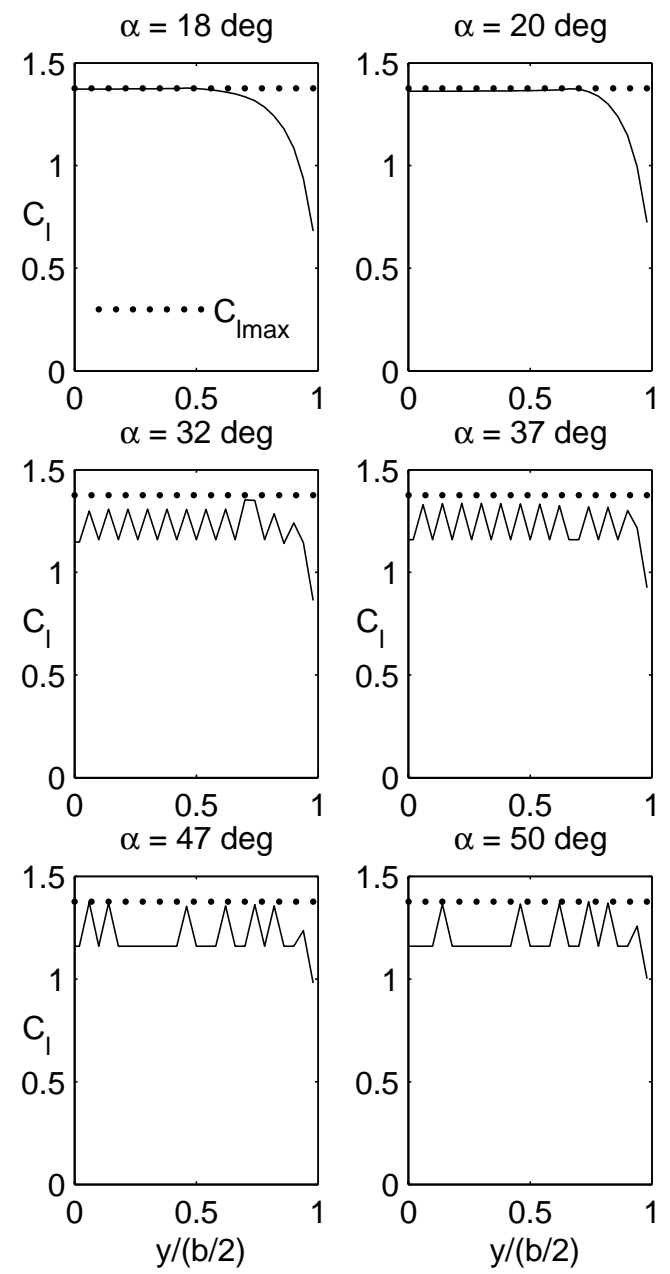

Figure 4.20: Spanwise $C_{l}$ distribution predicted for a rectangular wing of aspect ratio 9, using a NACA 4415 airfoil at Reynolds number of 0.75 million from scheme 1. 

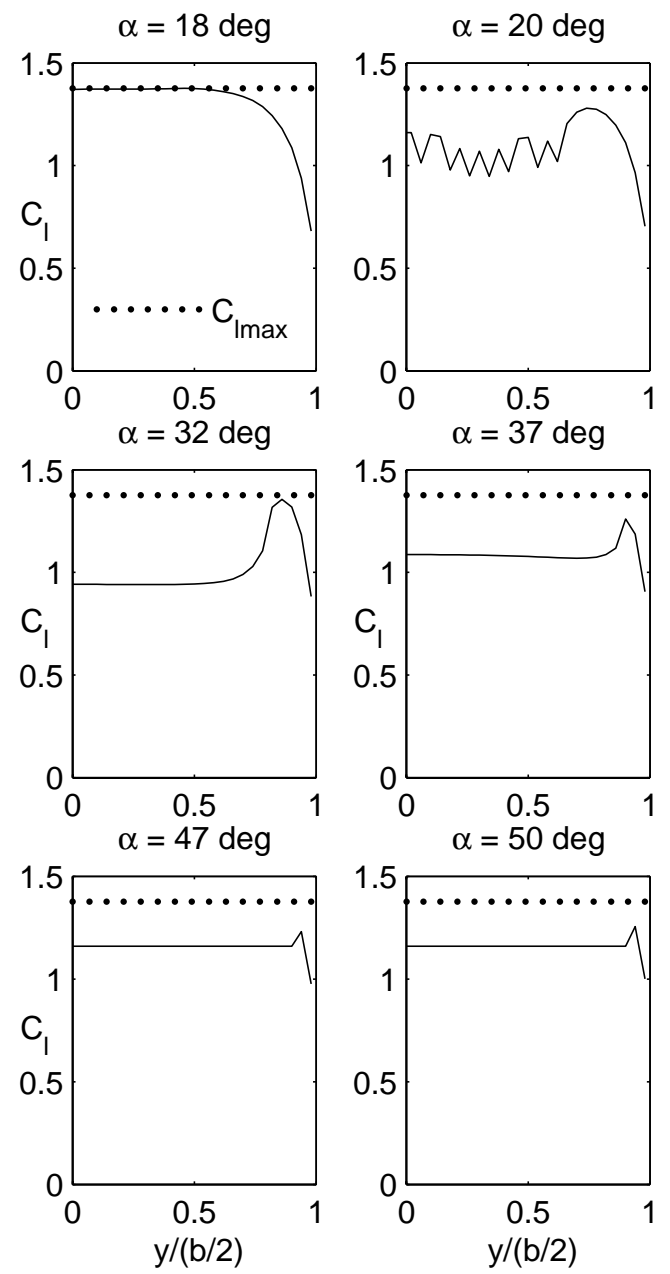

Figure 4.21: Spanwise $C_{l}$ distribution predicted for a rectangular wing of aspect ratio 9, using a NACA 4415 airfoil at Reynolds number of 0.75 million from scheme 2 . 


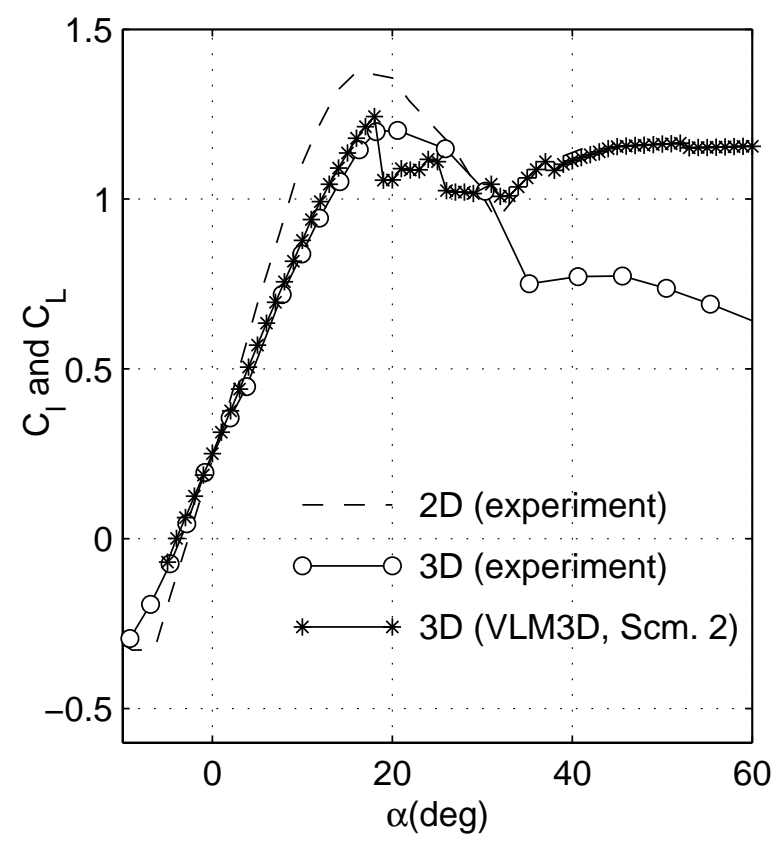

Figure 4.22: Wing $C_{L^{-}} \alpha$ predicted using scheme 2 for a rectangular wing of aspect ratio 6, using a NACA 4415 airfoil at Reynolds number of 0.75 million.
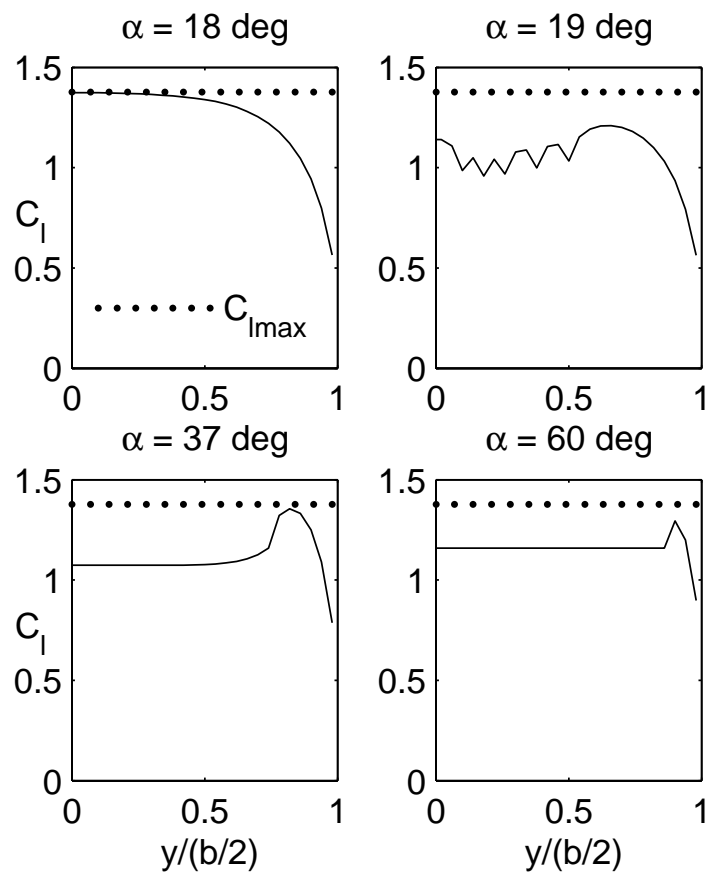

Figure 4.23: Spanwise $C_{l}$ distribution predicted for a rectangular wing of aspect ratio 6, using a NACA 4415 airfoil at Reynolds number of 0.75 million from scheme 2 . 


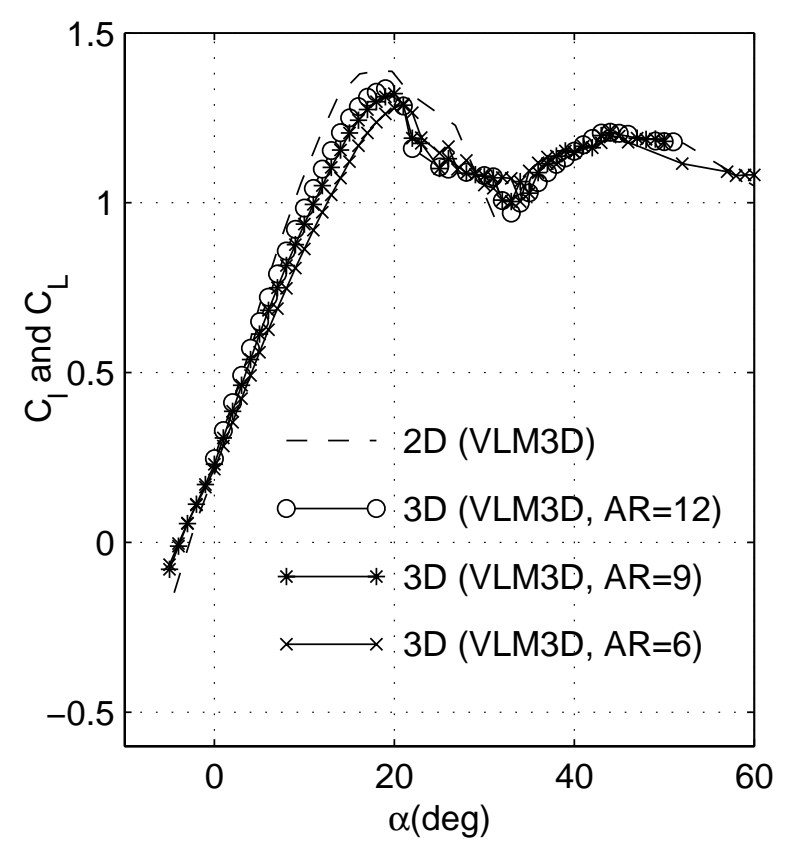

Figure 4.24: Wing $C_{L^{-}} \alpha$ predicted from VLM3D for rectangular wings of aspect ratios 12, 9 and 6 using a NACA 4415 airfoil at Reynolds number of 0.5 million.

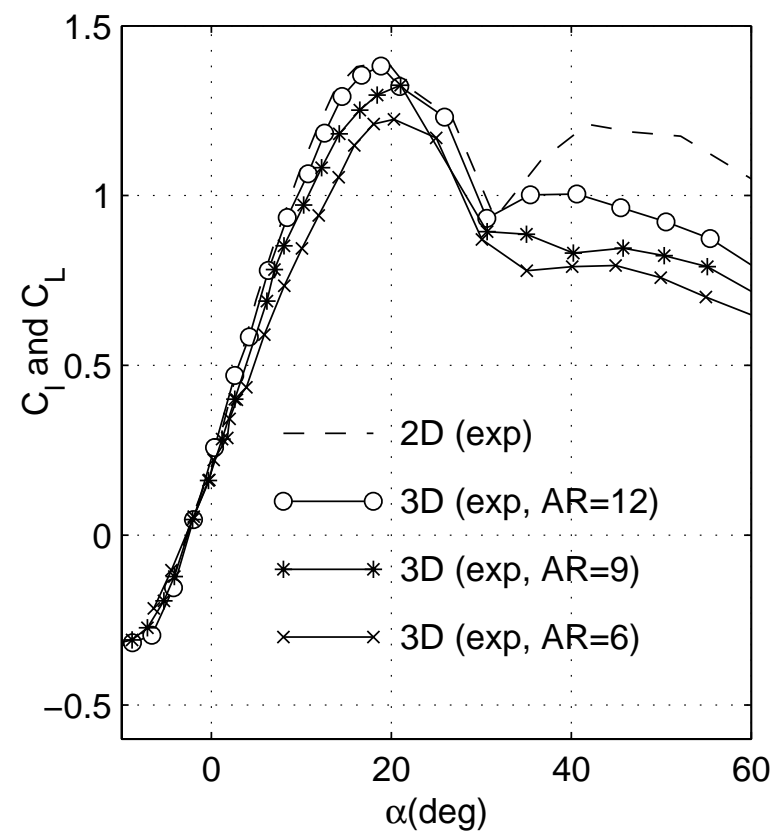

Figure 4.25: Wing $C_{L^{-}} \alpha$ predicted from experiment for rectangular wings of aspect ratios 12, 9 and 6 using a NACA 4415 airfoil at Reynolds number of 0.5 million. 


\subsubsection{Summary}

Having made an in-depth study of the results predicted by schemes 1 and 2, it is found that Scheme 2 is more robust. The spanwise section $C_{l}$ distributions predicted by scheme 1 exhibit substantial undesirable sawtooth behavior. The spanwise section $C_{l}$ distributions predicted by Scheme 2, on the other hand, are devoid of such oscillations for most of the cases studied. Scheme 1 also has far

more convergence problems than Scheme 2. Scheme 2, is therefore found to be better suited for post-stall prediction purposes and results presented henceforth will only be from Scheme 2 . 


\subsection{Study of stall characteristics}

In this example, the change in stall characteristics with change in taper ratio is studied. For this purpose, four wings each of aspect ratio 10 and taper ratios of $0.3,0.5,0.75$ and 1.0 are studied. The right-side planform of the wing with taper ratio of 1.0 (rectangular wing), is shown in Fig. 4.26 and the right-side planforms of the wings of taper ratios of $0.3,0.5$ and 0.75 are as shown in Fig. 4.27. The airfoil used has the hypothetical lift curve as shown in Fig. 4.28. This lift curve is similar to those used by Sears ${ }^{4}$ and by Levinsky. ${ }^{6}$

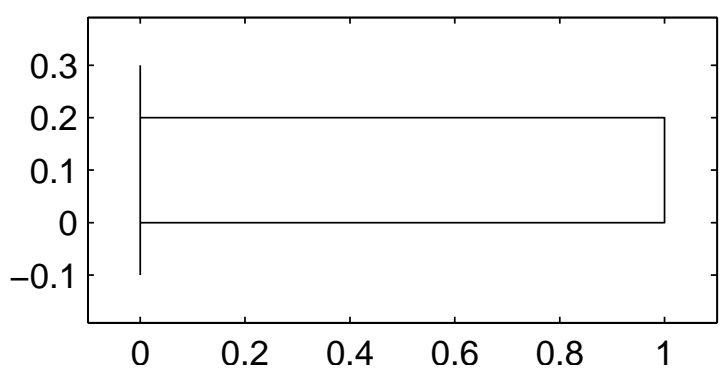

Figure 4.26: Planform of the rectangular wing (RHS shown) used in sec. 4.2.

The wing $C_{L^{-}} \alpha$ of the four wings from scheme 2 is shown in Fig. 4.29 and the spanwise section $C_{l}$ distributions from scheme 2 for the four wings for $\alpha$ of 10,17 , 18 and 24 deg are shown in Figs. 4.30, 4.31, 4.32 and 4.33 respectively. It is observed from the wing $C_{L^{-}} \alpha$ curves for the wings of taper ratios of $0.3,0.5$ and 0.75 that the wing $C_{L}$ increases as the $\alpha$ is increased to 17 deg. However, the rectangular wing stalls at the root at a slightly lower angle of attack of 16 deg. This behavior occurs because at a given $\alpha$, the rectangular wing has the largest section $C_{l}$ among all the wings in this study. This can be confirmed by examining the spanwise section $C_{l}$ distributions from scheme 2 for the four wings in Fig. 4.30 for $\alpha$ of $10 \mathrm{deg}$. At $10 \mathrm{deg}$, the section $C_{l}$ on the root of the wing of taper ratio of 1.0 is the largest and with decrease in taper ratio the section $C_{l} \mathrm{~s}$ on the roots of the other three wings decrease. This pattern is exactly reversed on the tips of 

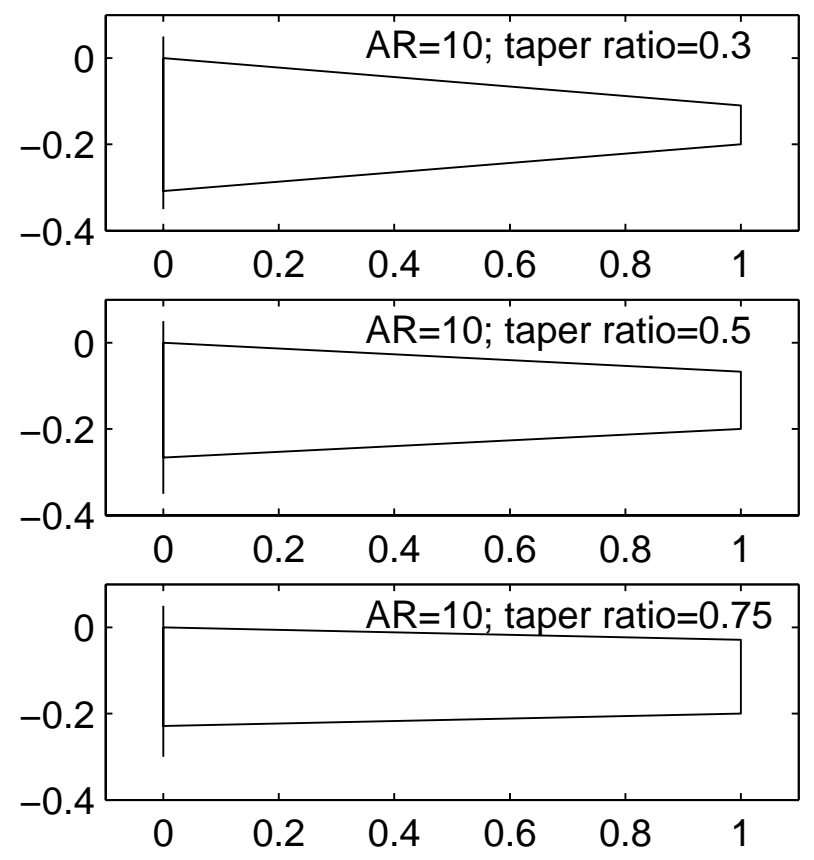

Figure 4.27: Planform of the tapered wings (RHS shown) used in sec. 4.2.

the four wings. The section $C_{l}$ in the vicinity of the tips of the wing of taper ratio of 1 is the least and with decrease in taper ratio the section $C_{l}$ on the outboard portions of the other three wings increase.

As the $\alpha$ is increased beyond $16 \mathrm{deg}$ the wing $C_{L}$ of the wing of taper ratio of 1 decreases sharply. This is because the root of this wing stalls as can be seen from the section $C_{l}$ distribution at $17 \mathrm{deg}$ for the wing of taper ratio of 1 in Fig. 4.31. At $17 \mathrm{deg}$, the section $C_{l}$ on the outboard portions of all the four wings is increasing. The outboard portion of the wing of taper ratio of 0.3 is closest to stall and with increase in taper ratio the section $C_{l}$ on the outboard portion of the other wings move farther away from $C_{L \max }$ as seen from Fig. 4.31. At 18 deg, large portions of the three wings of taper ratios of $0.3,0.5$ and 0.75 have stalled and the outboard portion of the wing of taper ratio of 1.0 is near stall as shown in Fig. 4.32. A small part of the inboard portion of the wings of taper ratios of $0.3,0.5$ and 0.75 is unstalled. For the wing of taper ratio 0.75 the stalled portion is located more inboard and for the wing of taper ratio 0.3 the stalled portion is 


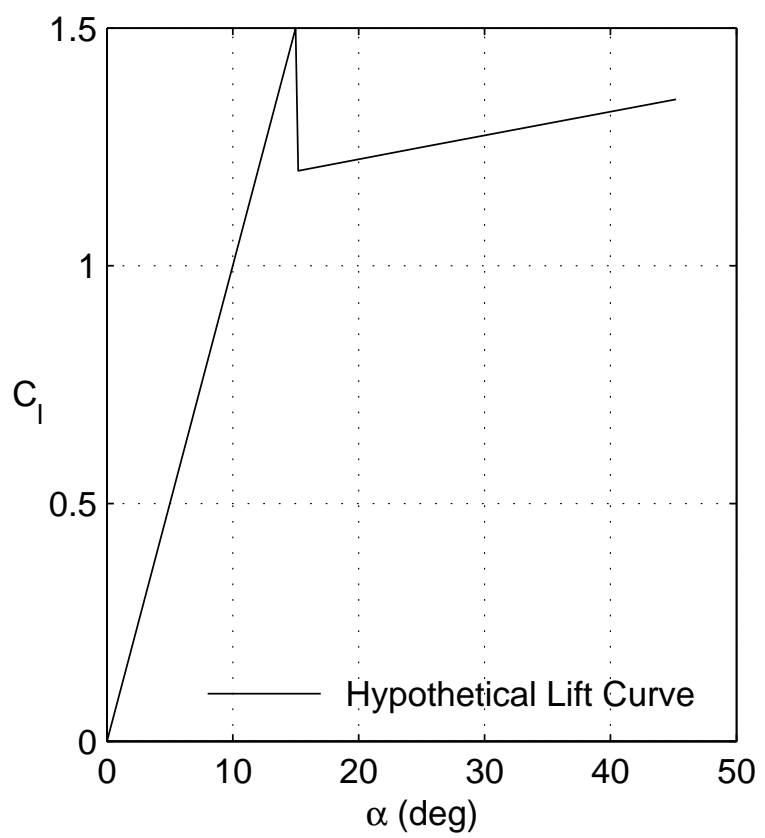

Figure 4.28: Airfoil lift curve used in sec. $4.2-4.5$.

located nearest to the tips as seen from Fig. 4.32. Hence, with increase in taper ratio the stalled regions are located closer to the root. At 24 deg the root stalls for the three wings of taper ratios of $0.3,0.5$ and 0.75 and the tips of the wing of taper ratio of 1.0 stall as shown in Fig. 4.33.

The pre-stall wing $C_{L}$ from scheme 2 does not show any noticeable differences between the four wings as shown in Fig. 4.29. This is because the four wings are of the same aspect ratio. However, the four wings have different stall characteristics. The trends in stall behavior with taper ratio predicted by the current method are very similar to those seen in experiments. In summary, it is observed that stall begins at the root of the rectangular wing and at the outboard portion on the tapered wings. More of the wing tips stall for the wing of taper ratio of 0.3 than for the taper ratio of 0.75 . 


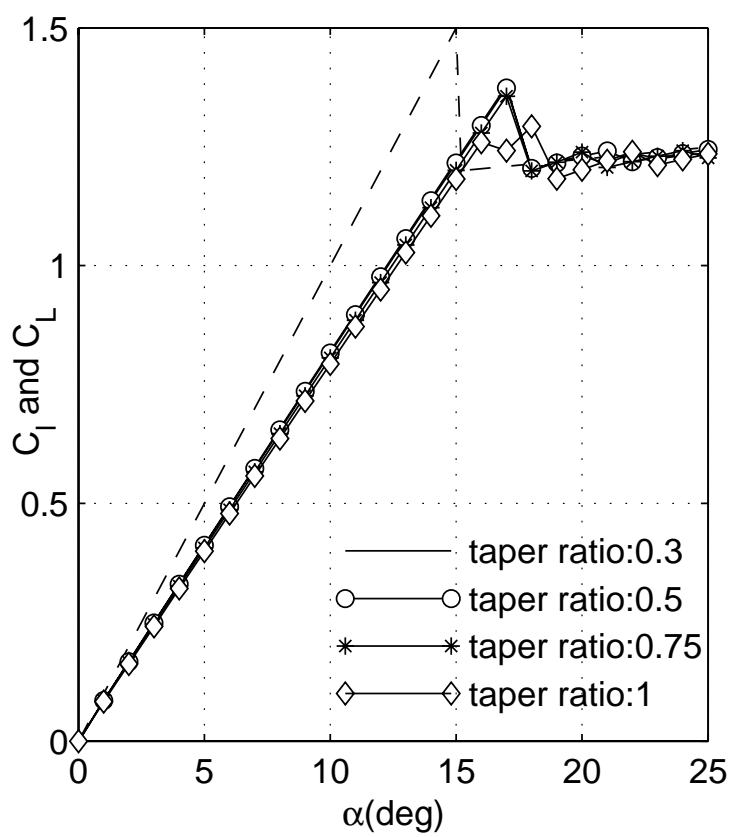

Figure 4.29: Wing $C_{L^{-}} \alpha$ for wings of different taper ratios, each of aspect ratio 10 using scheme 2 .

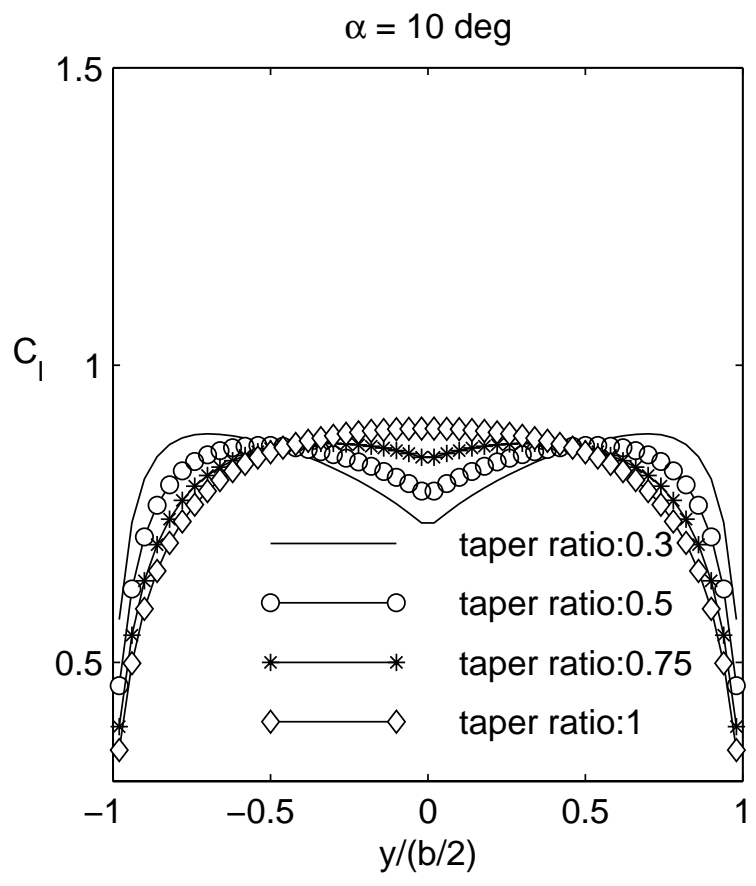

Figure 4.30: Change in the spanwise section $C_{l}$ distribution with taper at $\alpha$ of 10 deg using scheme 2 . 


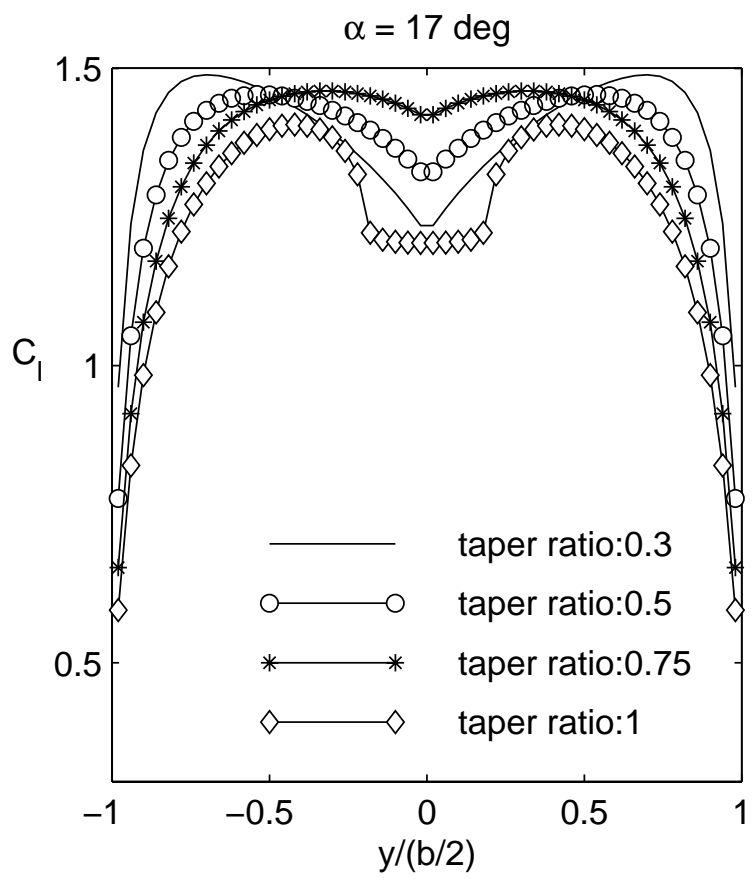

Figure 4.31: Change in the spanwise section $C_{l}$ distribution with taper at $\alpha$ of 17 deg using scheme 2.

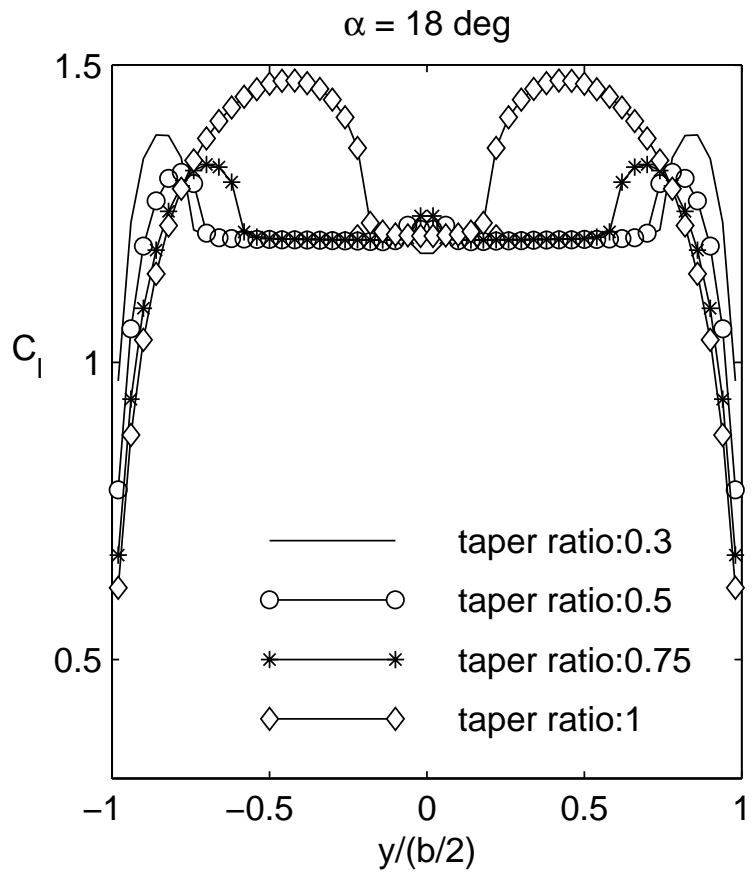

Figure 4.32: Change in the spanwise section $C_{l}$ distribution with taper at $\alpha$ of 18 deg using scheme 2. 


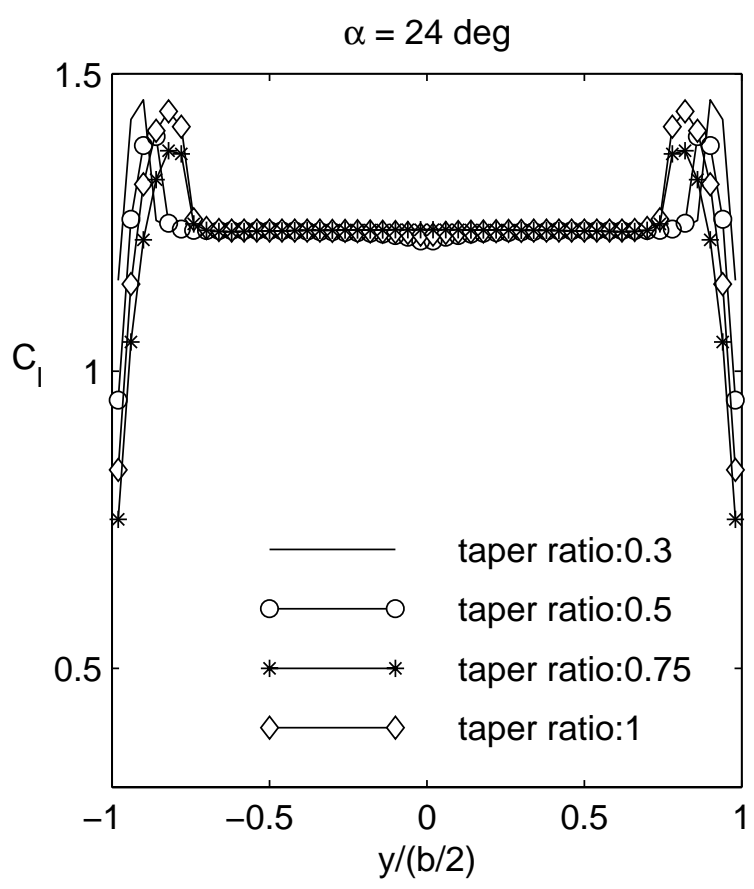

Figure 4.33: Change in the spanwise section $C_{l}$ distribution with taper at $\alpha$ of 24 deg using scheme 2.

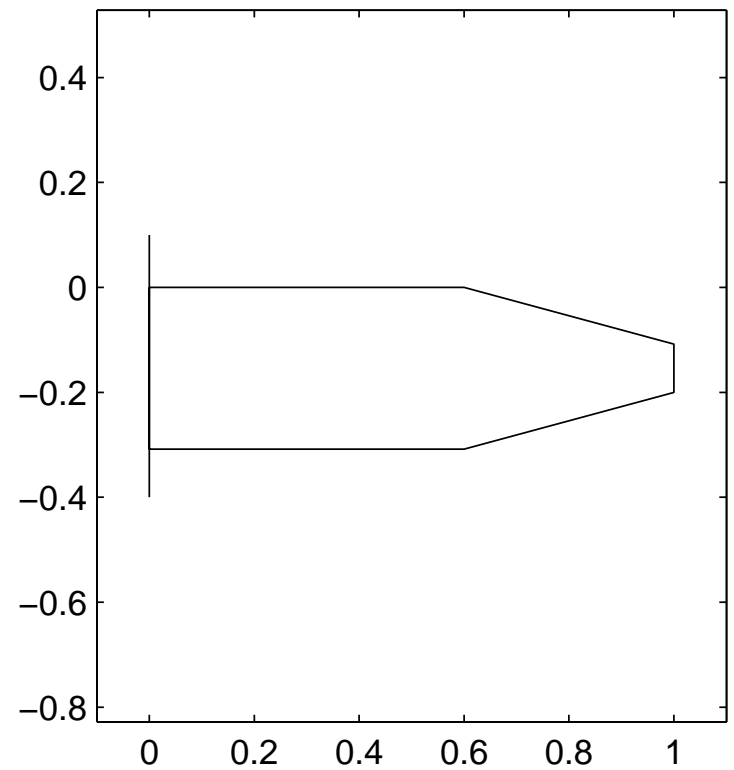

Figure 4.34: Planform of the part-tapered wing (RHS shown). 


\subsubsection{Stall Characteristics of a Part-Tapered Wing}

As seen in the examples so far, the rectangular wing has a tendency to stall first at the root which provides stall warning and greater aileron control. A tapered wing, on the other hand, provides a decrease in induced drag and good structural properties. A highly tapered wing has a tendency to stall first slightly at the outboard portion of the wing. A good compromise for a low-speed aircraft is a combination of both rectangular and tapered planform shapes. The rectangular inboard portion provides good stall characteristics and is cost effective. The tapered outboard portion decreases weight and increases aspect ratio. Therefore, in this example, such a planform is studied and the right-side of the planform is as shown in Fig. 4.34. The airfoil used has the hypothetical lift curve as shown in Fig. 4.28.

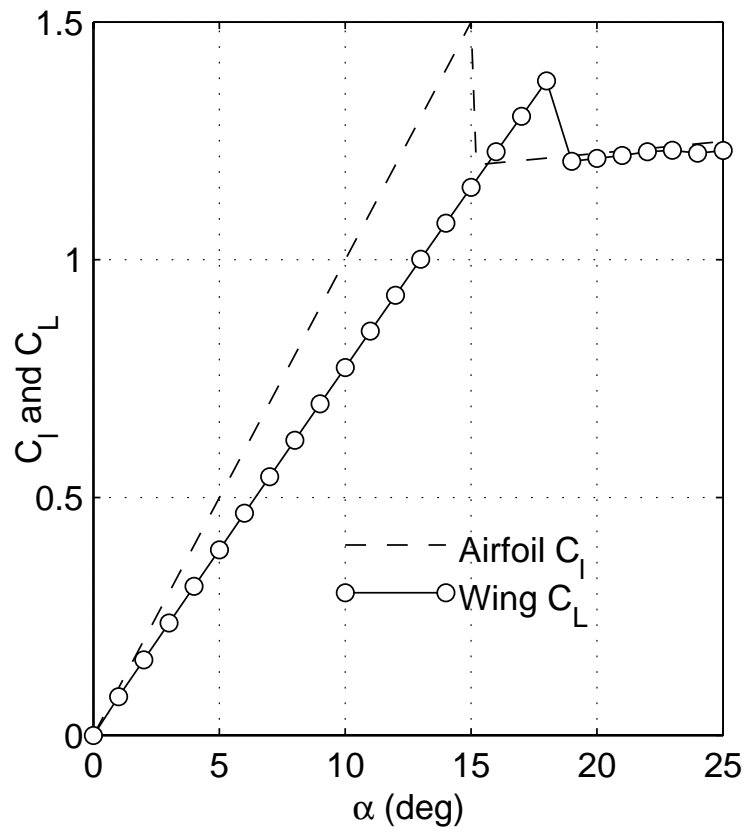

Figure 4.35: Lift curves for the part-tapered wing.

The wing $C_{L^{-}} \alpha$ for this planform from scheme 2 is as shown in Fig. 4.35 and the spanwise section $C_{l}$ distributions for $\alpha$ of 18, 19, 23 and 25 degrees are as 

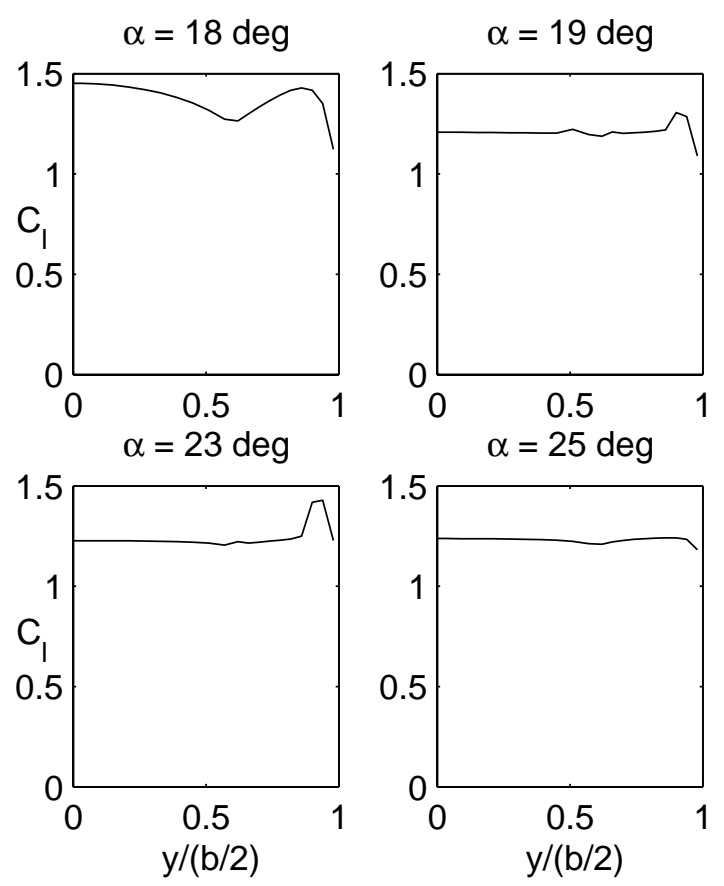

Figure 4.36: Spanwise $C_{l}$ distributions for the part-tapered wing.

shown in Fig. 4.36. It can be seen from Fig. 4.35 that as the $\alpha$ is increased to 18 deg the wing $C_{L}$ increases. At this condition the entire wing remains unstalled as the local section $C_{l}$ values are less than the $C_{l \max }$ of 1.5 . This can be confirmed by examining the spanwise section $C_{l}$ distribution from scheme 2 in Fig. 4.36 for $\alpha$ of $18 \mathrm{deg}$. As the $\alpha$ is increased to $19 \mathrm{deg}$ the inboard portion of the wing and a part of the outboard portion near the tips of the wing stall as can be seen from the spanwise section $C_{l}$ distribution for $\alpha$ of 19 deg in Fig. 4.36. As a result the wing $C_{L}$ also drops as the $\alpha$ is increased from 18 to $19 \mathrm{deg}$. At $23 \mathrm{deg}$ the $C_{l}$ on a small portion near the wing tips is near stall as can be seen from the section $C_{l}$ distribution at $\alpha$ of $23 \mathrm{deg}$ in Fig. 4.36. At $25 \mathrm{deg}$, almost the entire wing is stalled except for a small section in between the wing tips and the wing root. Such a part-tapered wing planform allows for tailoring of the stall characteristics by adjusting the location of the planform break and the taper ratio. 


\subsection{Wing-Tail Configuration}

This example illustrates the effectiveness of the method for a multiple liftingsurface configuration. In this example, results are presented for a high aspect ratio $(\mathrm{AR}=10)$ constant-chord wing geometry with a conventional aft tail. The planform for the wing-tail configuration is shown in Fig. 4.37. For this example, a single airfoil is assumed for both the wing and tail. The airfoil lift curve is the hypothetical lift curve as shown in Fig. 4.28.

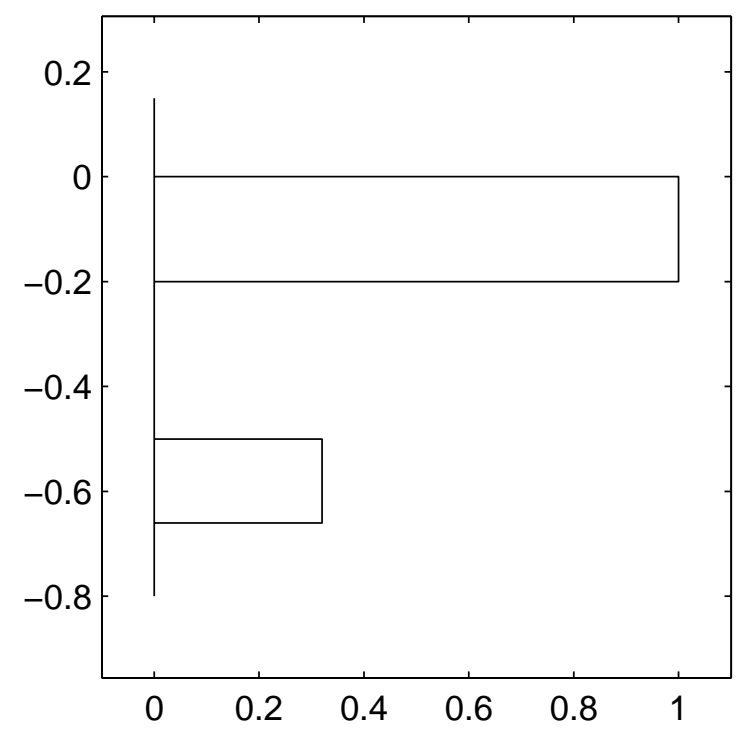

Figure 4.37: Planform of the wing-tail configuration (RHS shown).

Figure 4.38 shows the predicted $C_{L^{-}} \alpha$ curve for the wing-tail configuration with scheme 2 and starting $\delta_{1}$ values of -25 for all sections at each $\alpha$. Also shown in the same figure, is the airfoil $C_{l^{-}} \alpha$ curve for comparison. Figure 4.39 shows the $C_{L, w}$ and $C_{L, t}$ as a function of $\alpha$ along with the wing-tail total $C_{L^{-}} \alpha$ curve. It must be mentioned that the tail $C_{L, t}$ in this plot is nondimensionalized with reference to the tail area. The spanwise $C_{l}$ distributions are shown in Fig. 4.40 for $\alpha$ values of $10,15,18$, and $23 \mathrm{deg}$. These values were chosen for illustrating the stall behavior. 


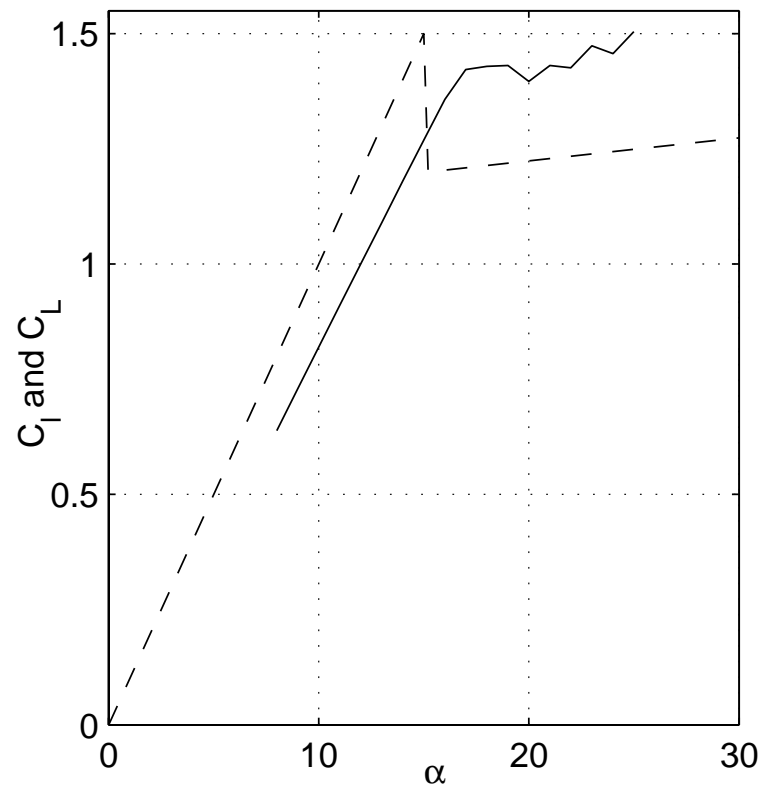

Figure 4.38: Lift curves for the wing-tail configuration.

From Fig. 4.38, it is seen that as the $\alpha$ is increased to $17 \mathrm{deg}$, the $C_{L}$ continues to increase. At these conditions, the entire wing remains unstalled as the local section $C_{l}$ values are less than $C_{l \max }$ of 1.5 . This result can be confirmed by examining the spanwise section $C_{l}$ distributions for the wing at $\alpha$ of 10 and 15 deg in Fig. 4.40. At $\alpha$ of 17 deg, the inboard portion of the wing is close to stall. As the $\alpha$ is increased from $17 \mathrm{deg}$ to $18 \mathrm{deg}$, the inboard portion of the wing stalls as seen in the spanwise $C_{l}$ plot for the wing at $\alpha$ of $18 \mathrm{deg}$. As a result, the wing $C_{L^{-}} \alpha$ curve shows a stall break at $\alpha$ of $17 \mathrm{deg}$. Although the inboard portion of the wing is stalled, the $C_{l}$ on the outboard portion continues to increase with $\alpha$; and this causes the $C_{L}$ to increase again between $20 \mathrm{deg}$ and $25 \mathrm{deg}$. Beyond $\alpha$ of $20 \mathrm{deg}$, the spanwise extent of the stalled region increases with increasing $\alpha$ as shown in the spanwise $C_{l}$ plot at $\alpha$ of 23 deg. In this example, the tail remains unstalled over the entire $\alpha$ range considered.

Of particular interest when studying wing-tail configurations is the aircraft pitching moment about the CG. For this analysis, the CG was located to provide 


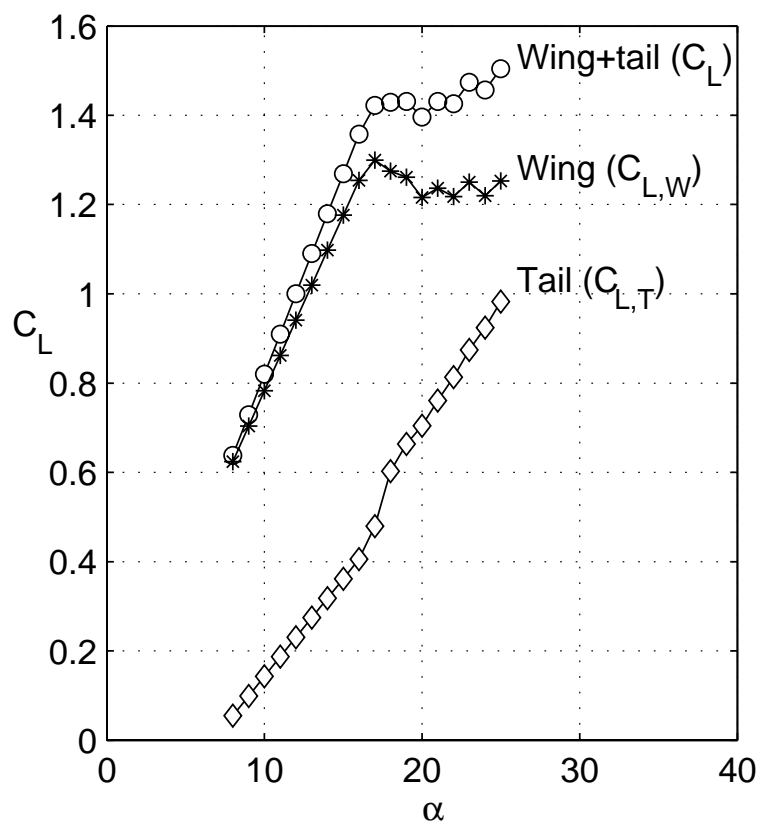

Figure 4.39: Individual contributions of the wing and tail to the total lift of the wing-tail configuration.

a static margin of $10 \%$ of the wing mean aerodynamic chord. A tail incidence of -5 deg was chosen and results in the aircraft being trimmed at $\alpha$ close to 13 deg. In other words, the $C_{M_{c g}}$ is nearly zero at $\alpha$ of $13 \mathrm{deg}$. Figure 4.41 shows the variation of the aircraft $C_{M c g}$ as a function of $\alpha$. As the $\alpha$ is increased, a distinct increase in the nose-down pitching moment (negative $C_{M c g}$ ) is seen at the onset of stall at $\alpha$ of $17 \mathrm{deg}$.

To examine the cause of this nose-down pitching moment at stall onset, it is instructive to study the individual contributions of the wing and the tail to the configuration lift curve as shown in Fig. 4.39. It can be seen that much of the contribution to the aircraft $C_{L^{-}} \alpha$-curve behavior can be traced to the characteristics of the wing lift curve. There is, however, a noticeable increase in the tail $C_{L, t}$ at the onset of wing stall at $\alpha$ of $17 \mathrm{deg}$. This increase in $C_{L, t}$ at stall onset contributes significantly to the increase in the nose-down pitching moment at stall. The origin of the sudden increase in $C_{L, t}$ at stall can in turn be traced to the 

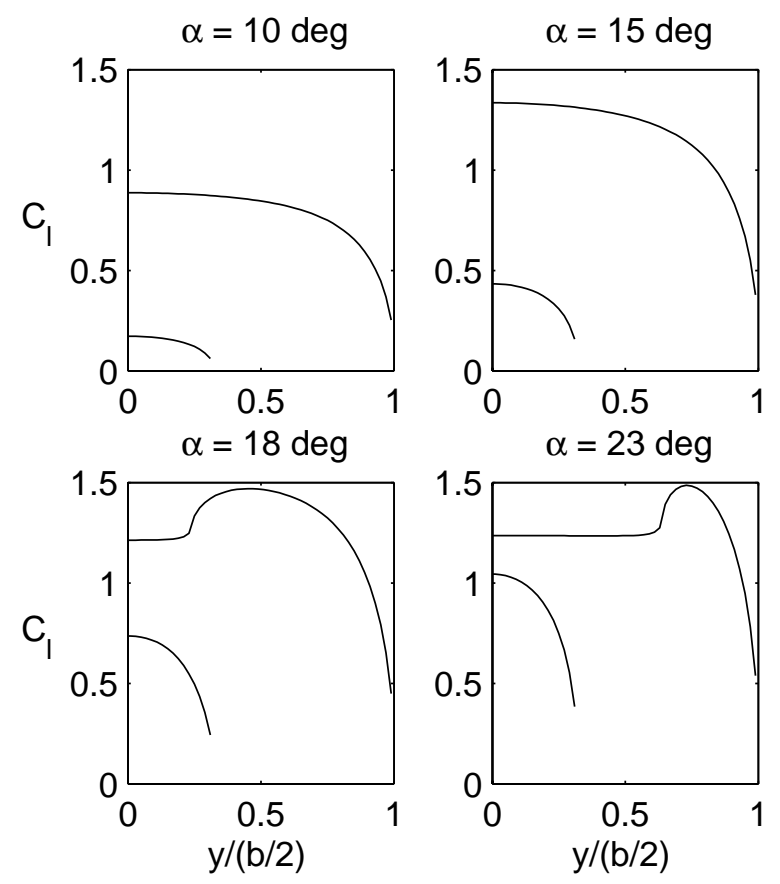

Figure 4.40: Spanwise $C_{l}$ distributions for the wing-tail configuration.

shape of the wing spanwise lift distribution at the onset of stall. As seen earlier in Fig. 4.40, the wing stall occurs at the root for $\alpha$ of 18 deg for this example. This loss in lift over the inboard portion of the wing results in a reduction in the downwash at the tail. In addition, it also results in a pair of vortices that cause an upwash at the tail. As a consequence of these two factors, there is a distinct increase in the $C_{L, t}$ at the onset of stall.

Thus, as illustrated by this simple example, a method that can predict the poststall characteristics of wing-tail configurations can provide important information for the study of aircraft longitudinal behavior at these post-stall conditions. 


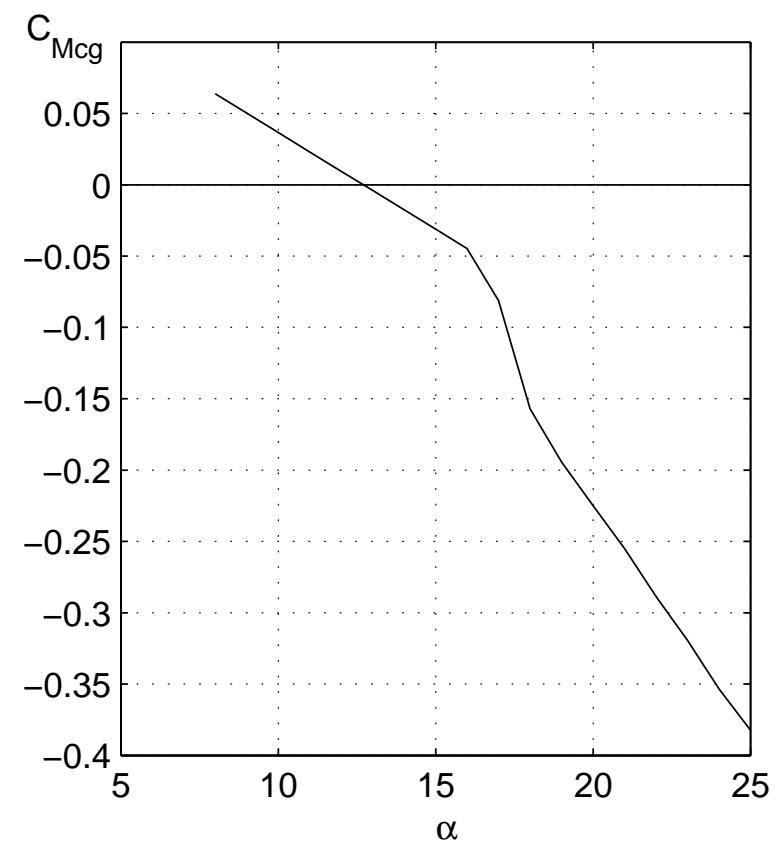

Figure 4.41: Pitching-moment curve for the wing-tail configuration. 


\subsection{Wing-Canard Configuration}

This example illustrates the effectiveness of the method for another multiple lifting-surface configuration, a high aspect ratio $(\mathrm{AR}=10)$ constant-chord wing geometry with a canard $(\mathrm{AR}=10)$. The planform for the wing-canard configuration is shown in Fig. 4.42. A single airfoil is assumed for both the wing and the canard. The airfoil lift curve is the hypothetical lift curve as shown in Fig. 4.28. An important criterion for acceptable stall behavior of such a configuration is that the canard stalls before the wing. Both the wing and canard have zero incidence. In this simple example, which is used only for illustration of the capability of the current method.

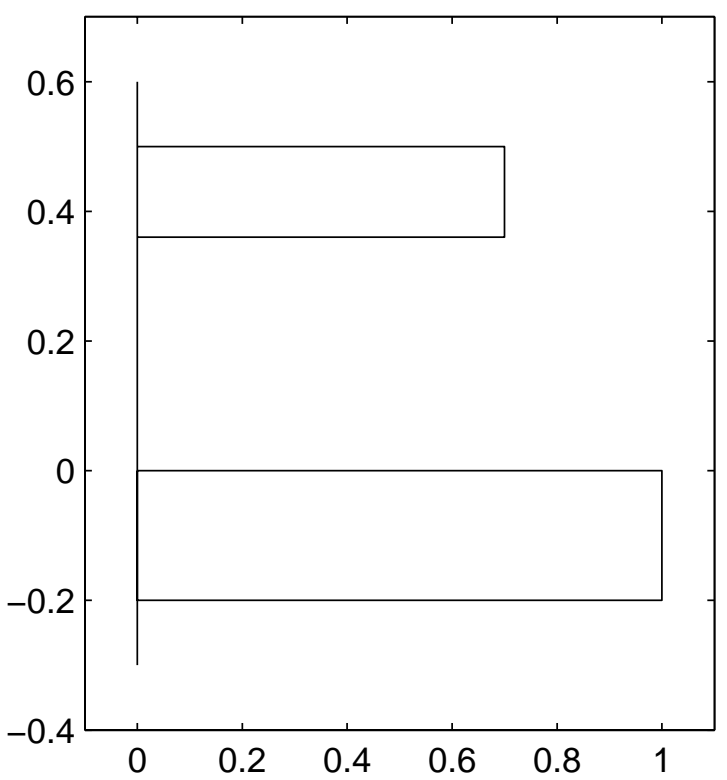

Figure 4.42: Planform of the wing-canard configuration (RHS shown).

Scheme 2 was used with starting $\delta_{1}$ values of 0 deg on all sections on both the wing and canard for the first $\alpha$ of the $\alpha$-sequence. The resulting configuration $C_{L^{-}} \alpha$ curve is shown in Fig. 4.43. The configuration $C_{L}$ is defined with reference to the wing planform area. Also shown in the same figure is the airfoil lift curve for comparison. The $\delta_{1}$ and $\delta_{2}$ values for each $\alpha$ is taken from the converged 


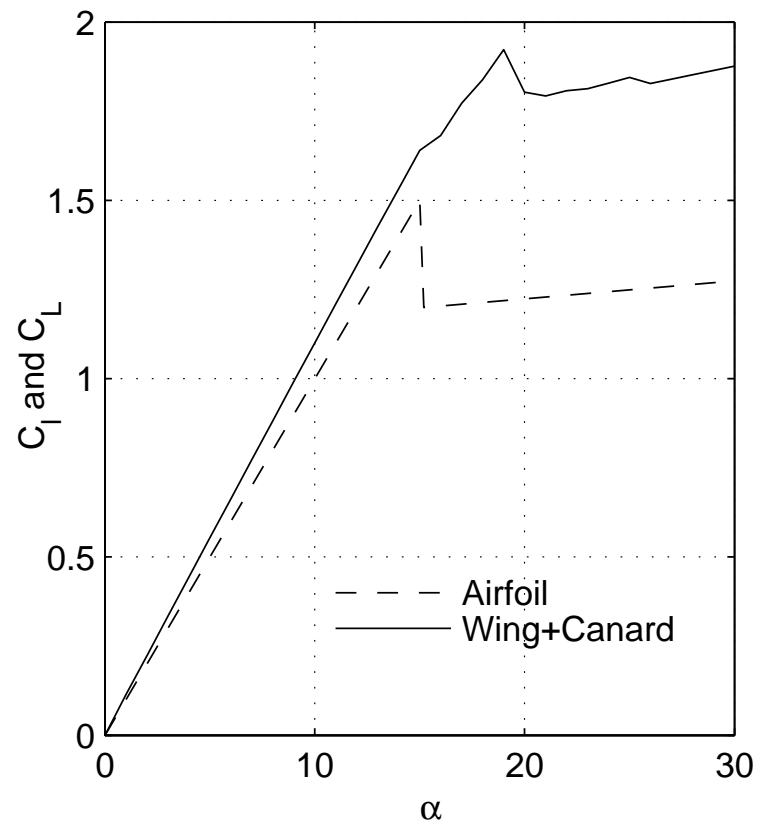

Figure 4.43: Lift curves of the wing-canard configuration.

values from the previous $\alpha$. The spanwise section $C_{l}$ distributions for the wing and canard are shown in Fig. 4.44 for $\alpha$ of 15, 16, 19, 20, 23 and 26 degrees. The values were chosen to explain the stall behavior. Figure 4.45 shows the lift curves of the wing and canard separately. Also plotted on the same figure are the wing-canard lift curve and the airfoil lift curve for comparison. The canard $C_{L}$ in this plot is defined with reference to the canard planform area.

From Fig. 4.43, it is seen that as the $\alpha$ is increased to $19 \mathrm{deg}$, the $C_{L}$ continues to increase. At these conditions, the entire wing is unstalled as the local section $C_{l}$ values are less than $C_{l \max }$ of 1.5 but the canard has stalled for $\alpha$ greater than 16 deg. From Fig. 4.45 it can be seen from the $C_{L^{-}} \alpha$ curve of the canard that as the $\alpha$ is increased to $15 \mathrm{deg}$ the canard remains unstalled. At $\alpha=15 \mathrm{deg}$ the canard is close to stall as can be seen from the section $C_{l}$ distribution of the canard at $\alpha=15 \mathrm{deg}$ in Fig. 4.44. As the $\alpha$ is increased just beyond 15 deg the root of the canard stalls as can be seen from the section $C_{l}$ distribution of the canard at $\alpha=16 \mathrm{deg}$ in Fig. 4.44. The wing $C_{L^{-}} \alpha$ on the other hand increases as the $\alpha$ is 

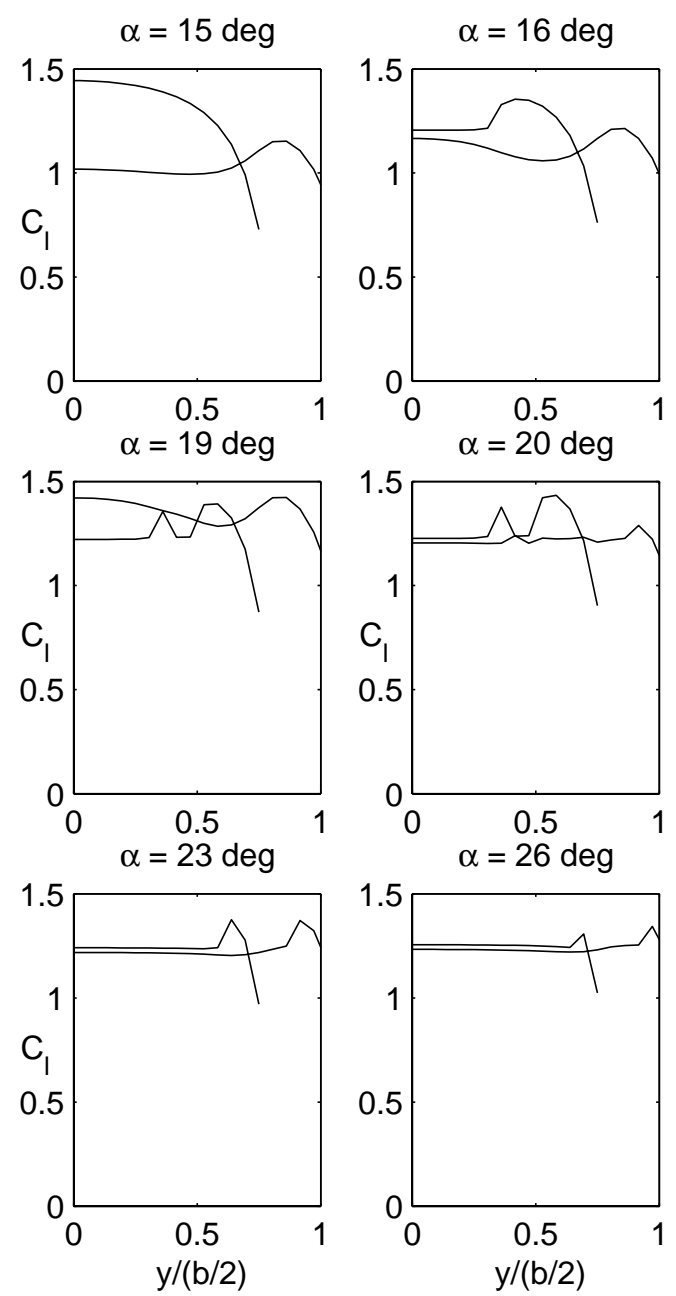

Figure 4.44: Spanwise $C_{l}$ distributions for the wing-canard configuration.

increased to $19 \mathrm{deg}$ as shown in Fig. 4.43. In such a configuration the inboard portion of the wing is under the influence of the downwash from the canard so that as the lift on the canard increases the downwash on the wing, which in turn causes reduced lift on the inboard portion of the wing. At $\alpha=15 \mathrm{deg}$ the canard is close to stall and the inboard portion of the wing has reduced lift but the lift on the outboard portion of the wing is increased because of the upwash from the canard. This is confirmed by the section $C_{l}$ distribution on the wing at $\alpha$ of 15 as shown in Fig. 4.44. At $16 \mathrm{deg}$, the canard stalls and therefore the downwash on the wing decreases. Also, two trailing vortices are shed from the edges of the 


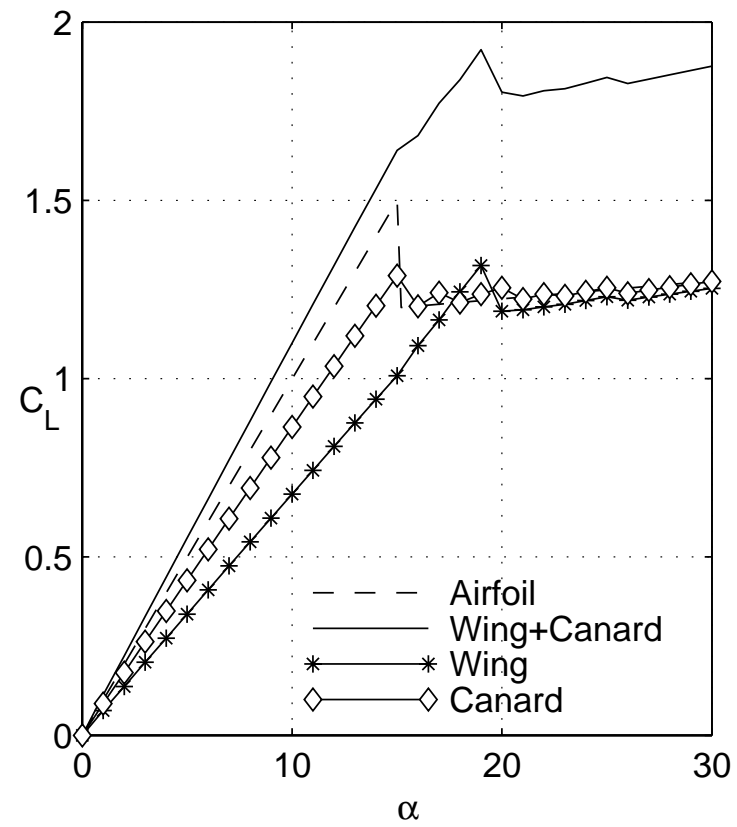

Figure 4.45: Individual contributions of the wing and canard to the total lift of the wing-canard configuration.

stalled portion of the canard that cause upwash on the inboard portion of the wing. As a result, the lift on the inboard portion of the wing now increases as seen from the section $C_{l}$ distribution on the wing at 16 as shown in Fig. 4.44. At 19 deg a part of the outboard portion of the wing and the inboard portion of the wing are close to stall. As the $\alpha$ is increased to 20 deg these portions of the wing stall as can be seen from the section $C_{l}$ distribution on the wing at 20 as shown in Fig. 4.44. As the $\alpha$ is increased beyond 20 deg most of the canard and the wing has stalled except for two small regions on the outboard portions as shown by the section $C_{l}$ distribution of the wing and canard at 23 deg in Fig. 4.44. At 26 deg both the wing and canard are stalled as shown by the section $C_{l}$ distribution of the wing and canard at 26 deg in Fig. 4.44. 


\subsection{Spanwise Asymmetry in the Initial Conditions}

In all the examples presented so far, the starting distribution of $\delta_{1}$ and $\delta_{2}$ was assumed to be a constant along the wing span. The spanwise section $C_{l}$ distributions obtained for these cases was symmetric. As stated before, while in the past, some researchers ${ }^{8,9}$ have observed no asymmetric lift distributions for symmetric flight conditions even when the iterations were started with asymmetric initial conditions, other researchers ${ }^{3,4,6}$ have observed asymmetric lift distributions for perfectly symmetric starting lift distributions. The aim of this example is, therefore, to investigate the presence of any asymmetry in the final converged lift distribution when the starting conditions(distribution of $\delta_{1}$ and $\delta_{2}$ along wing span) are asymmetric.

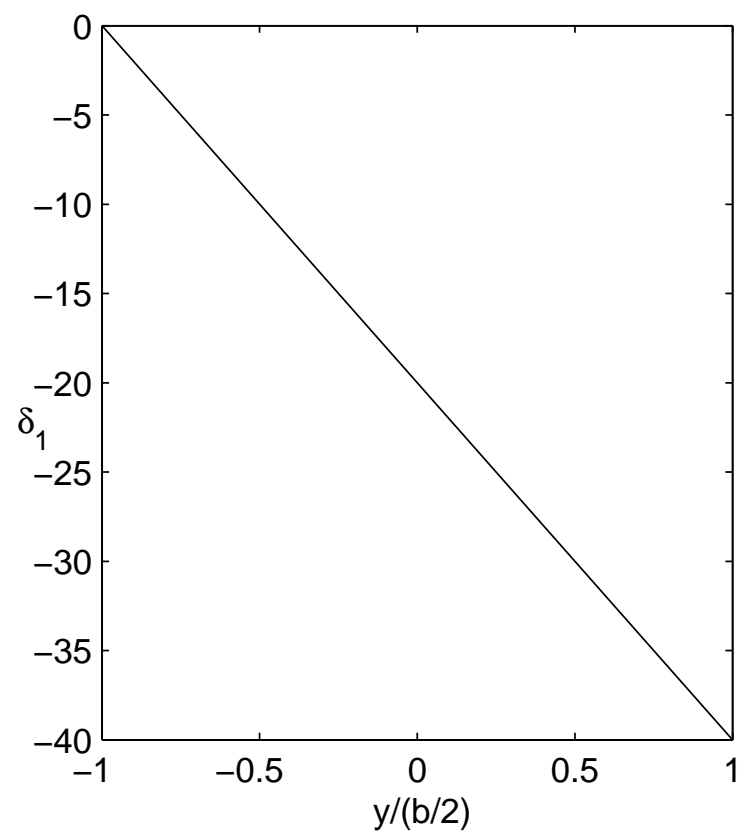

Figure 4.46: Initial $\delta_{1}$ distribution along wing span.

The right-side planform of the rectangular wing used in this example is shown in Fig. 4.26. The airfoil used has the hypothetical lift curve as shown in Fig. 4.28. 


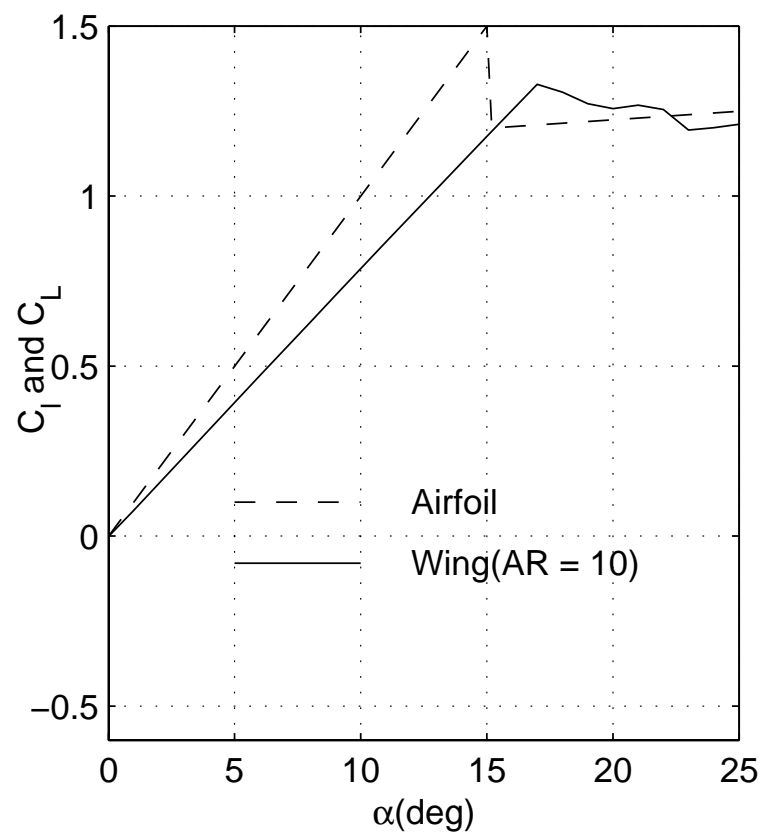

Figure 4.47: Wing $C_{L}$ with an initial asymmetric distribution of $\delta_{1}$.

Figure 4.46 shows the starting distribution of $\delta_{1}$ along wing span. The starting distribution of $\delta_{2}$ along wing span is constant and in this example it is assumed to be zero. An $\alpha$-sequence of $0-25 \mathrm{deg}$ is used, and for each $\alpha$, the starting values of $\delta_{1}$ are set as shown in Fig. 4.46 and the starting values of $\delta_{2}$ are set to zero along the wing span. The converged wing $C_{L^{-}} \alpha$ using this starting distribution of $\delta_{1}$ and $\delta_{2}$ is shown in Fig. 4.47. Figure 4.48 shows the final converged spanwise section $C_{l}$ for $\alpha$ of $10,15,20$ and 25 degrees. Using a tolerance of 0.001 , the number of iterations required to converge is 281 for $\alpha$ of $10 \mathrm{deg}, 289$ for $\alpha$ of $15 \mathrm{deg}, 222$ for $\alpha$ of $20 \mathrm{deg}$ and 142 for $\alpha$ of $25 \mathrm{deg}$. As can be seen in Fig. 4.48 there is no asymmetry in the spanwise section $C_{l}$ distributions although the starting distribution of $\delta_{1}$ is asymmetric. However, there is asymmetry in the spanwise section $C_{l}$ distribution after the first iteration, as shown in Fig. 4.49. The asymmetry is seen to be considerably diminished after 100 iterations, as shown in Fig. 4.50. The distribution of $\delta_{1}$ also shows similar behavior. The final converged distribution of $\delta_{1}$ along wing span does not show any asymmetry as shown in Fig. 4.51. However, 
the distribution of $\delta_{1}$ after the first and 100 iterations is asymmetric as shown in Figs. 4.52 and 4.53.
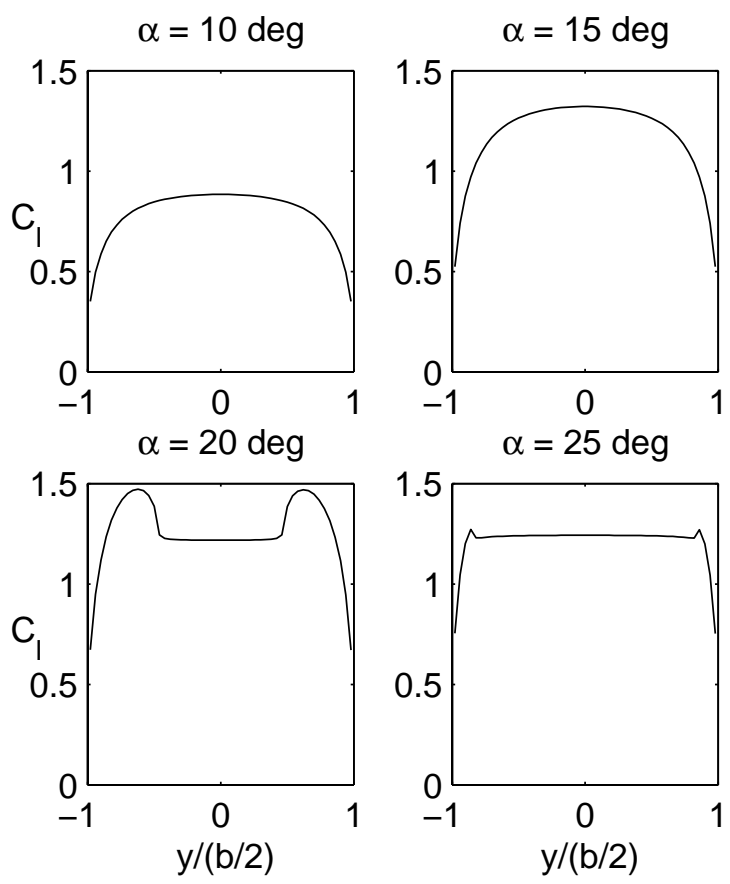

Figure 4.48: Final converged spanwise section $C_{l}$ with an initial asymmetric distribution of $\delta_{1}$.

The reason for the marked asymmetry in the spanwise section $C_{l}$ distributions after the first and after 100 iterations and symmetry in the final converged solutions can be traced to the distribution of the local-section effective angle of attack along span. The local-section effective angles of attack are directly related to $\delta_{1}$ and $\delta_{2}$ as shown in eqn. 3.7. The spanwise distributions of the local effective angle of attack, $\alpha_{\text {eff }}$ after 1 iteration, 100 iterations and after convergence are shown in Figs. 4.54, 4.55 and 4.56 respectively. As with the spanwise distribution of $\delta_{1}$ and $C_{l}$, the final converged solution of the $\alpha_{\text {eff }}$ also is not asymmetric but the solutions after the first and 100 iterations show asymmetry. 

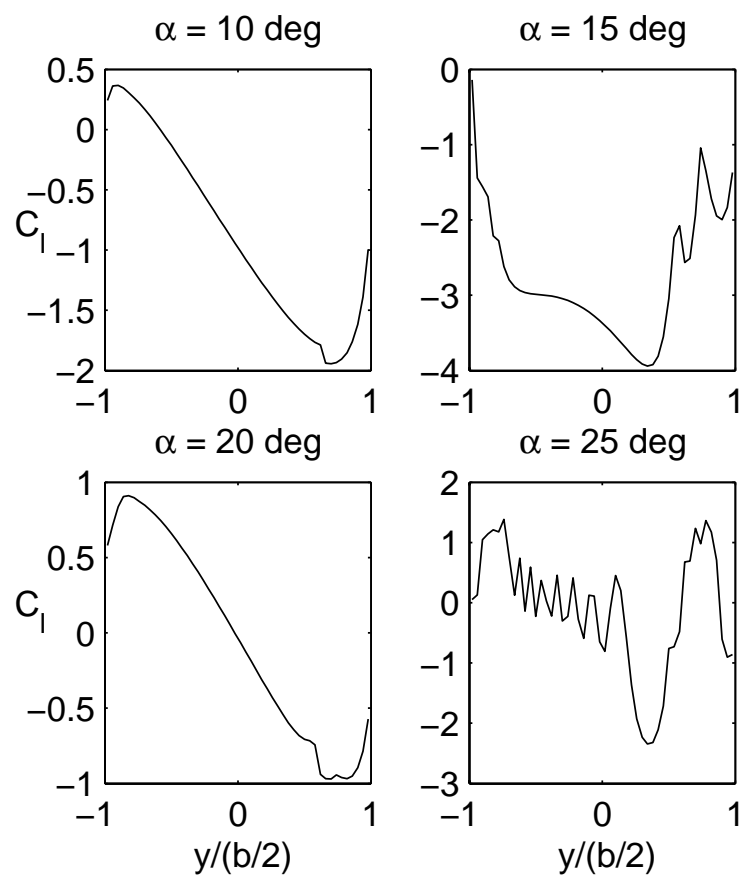

Figure 4.49: Spanwise section $C_{l}$ with an initial asymmetric distribution of $\delta_{1}$ after 1 iteration.
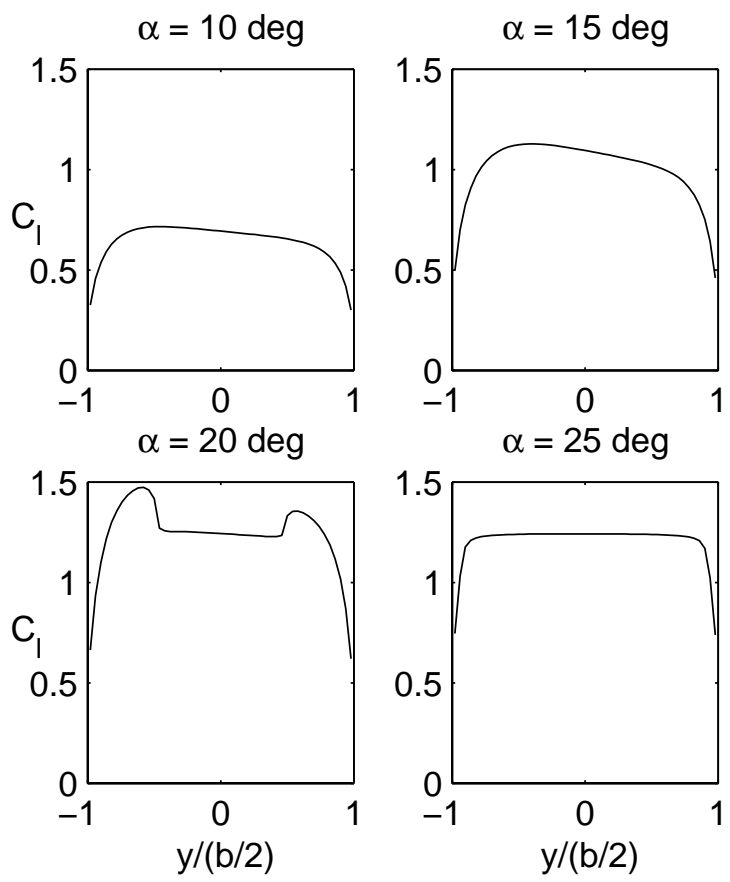

Figure 4.50: Spanwise section $C_{l}$ with an initial asymmetric distribution of $\delta_{1}$ after 100 iterations. 
Therefore, using VLM3D no asymmetry in the final converged lift distributions are observed even when the starting distribution of $\delta_{1}$ is asymmetric. The spanwise lift distributions, the spanwise $\delta_{1}$ distributions and the spanwise $\alpha_{\text {eff }}$ distribution however, start off showing marked asymmetry but with the increase in the number of iterations, the asymmetry progressively reduces and the final converged solution is symmetric.
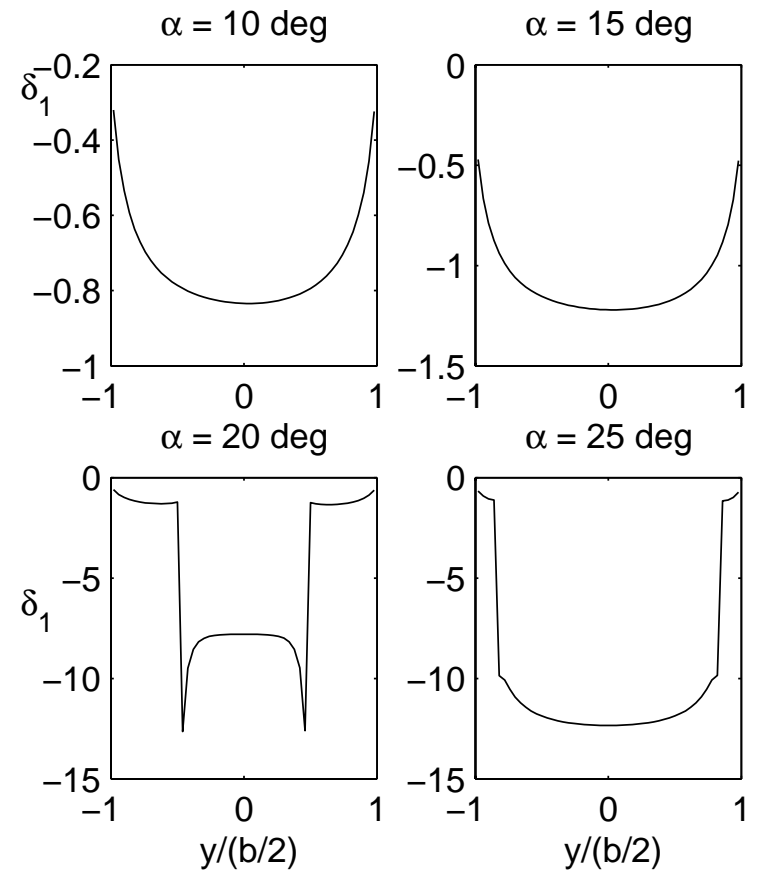

Figure 4.51: Final converged spanwise $\delta_{1}$ distributions. 

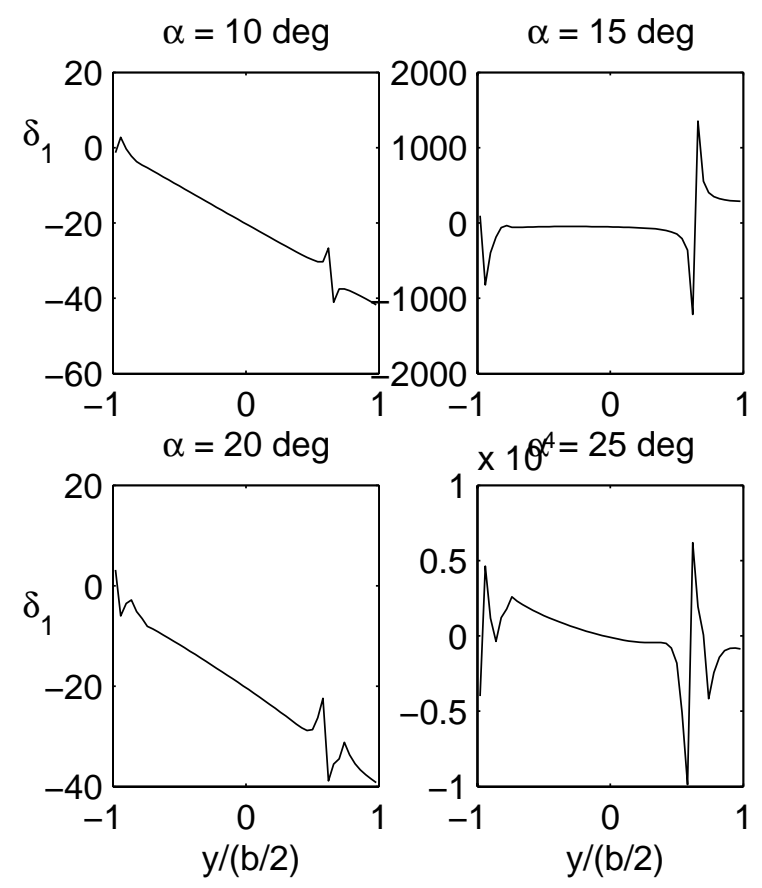

Figure 4.52: Spanwise $\delta_{1}$ distributions after 1 iteration.
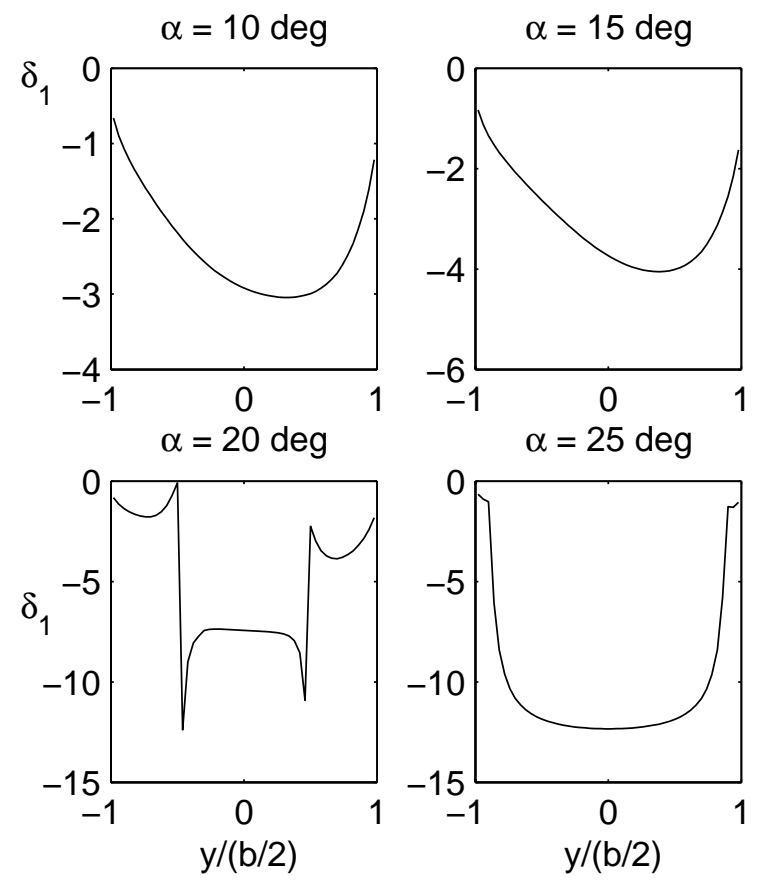

Figure 4.53: Spanwise $\delta_{1}$ distributions after 100 iterations. 

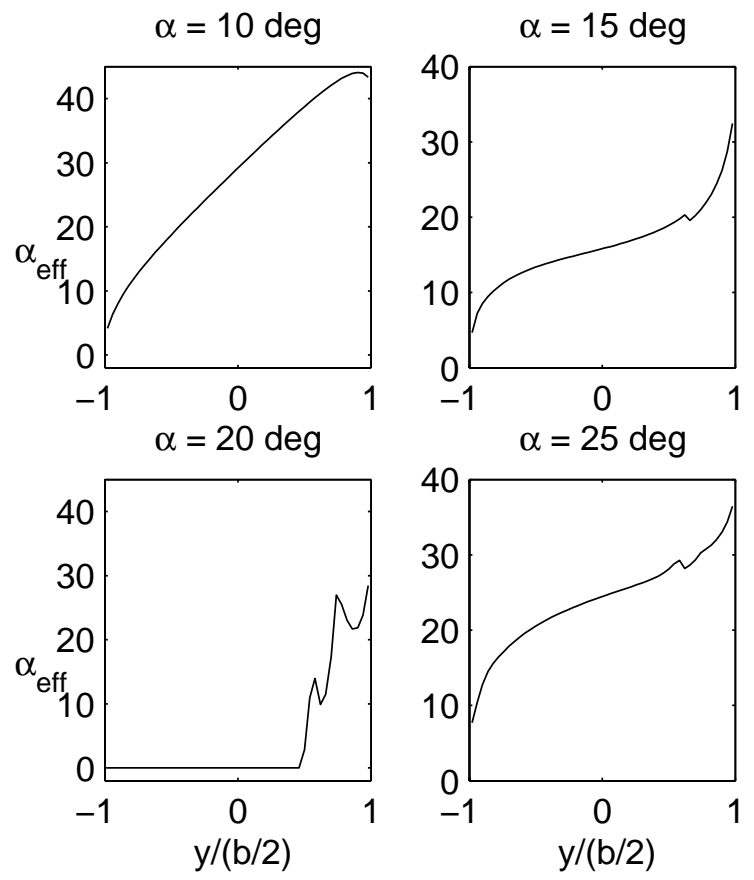

Figure 4.54: Spanwise distributions of $\alpha_{e f f}$ after 1 iteration.
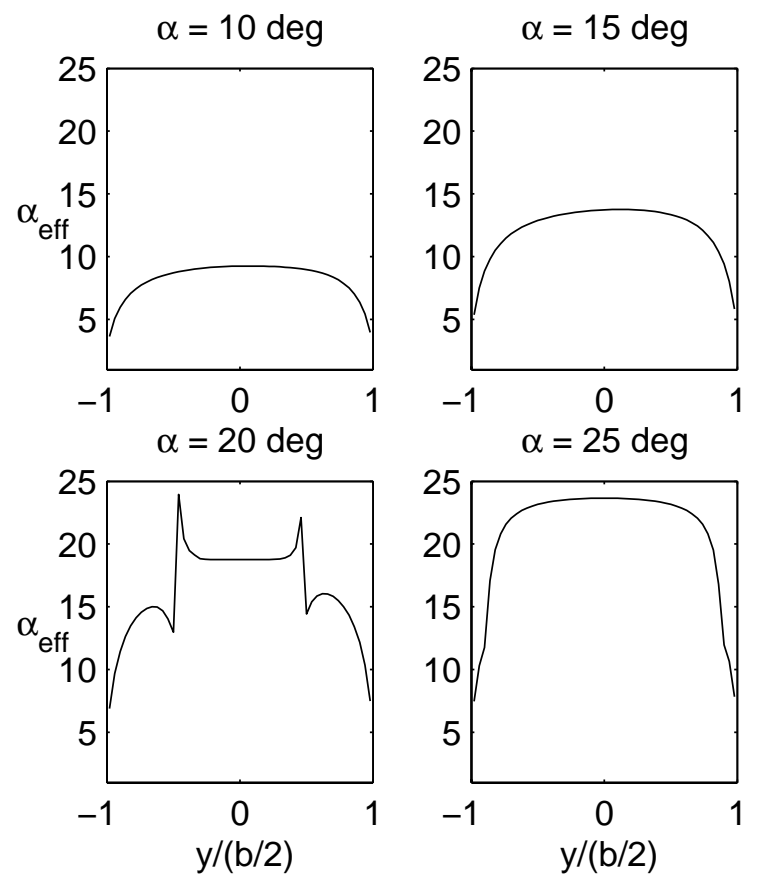

Figure 4.55: Spanwise distributions of $\alpha_{e f f}$ after 100 iterations. 

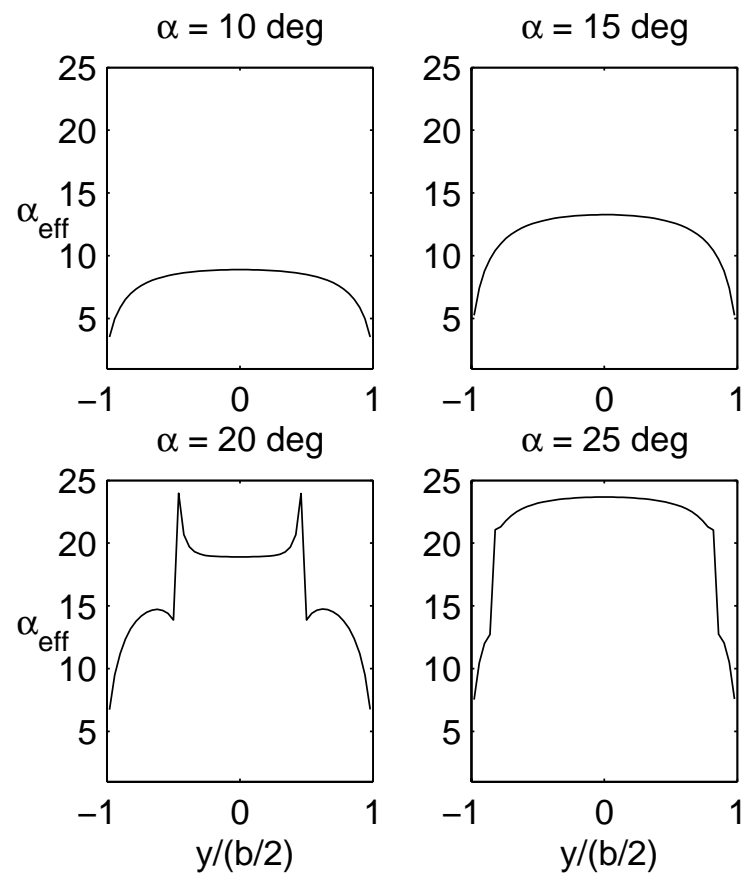

Figure 4.56: Final converged spanwise distributions of $\alpha_{e f f}$. 


\section{Chapter 5}

\section{Conclusions}

A decambering approach has been developed for the post-stall prediction of multiplelifting-surface configurations using known section data. The approach accounts for the boundary-layer separation effects on each section of each lifting surface in an iterative fashion. This approach is implemented using a multi-dimensional Newton iteration in a vortex-lattice method using two schemes, labeled scheme 1 and scheme 2. The two schemes differ in the procedure for the computation of the residuals. Scheme 2 is found to be more robust and its predictions more acceptable. A key feature of scheme 2 is that it takes into consideration the change in the effective $\alpha$ of a section when a small perturbation is made to the decambering at that section. As a consequence, the target $C_{l}$ for computing the residual is determined using the intersection(s) of a "trajectory line" and the airfoil lift curve. This trajectory line, which is computed for each section at every step of the iteration, is the line along which the point represented by the $C_{l}$ and the effective $\alpha$ at a section moves due to a perturbation to the decambering at that section.

A key outcome of determining the target $C_{l}$ using the intersection(s) of the trajectory line with the airfoil $C_{l^{-}} \alpha$ curve is that certain sections that are operating close to stall may end up with multiple intersections at high angles of attack. Scheme 2, thus, brings to light the existence of multiple solutions right during the iteration process. Earlier approaches were able to identify the existence of multiple 
solutions only as a result of obtaining multiple converged solutions with different initial conditions in the iteration procedure. Scheme 2, therefore, brings new insight to the iterative solution of wing flows using known airfoil characteristics. A special logic has been developed for sections with multiple intersections in order to choose a solution from one of the multiple choices for the target $C_{l}$.

The current method has been used for the analysis of several example single and multiple wings. The first set of examples have been used for comparison with experimental data of Naik and Ostowari. ${ }^{17}$ This experimental dataset includes lift characteristics of the NACA 4415 airfoil at several Reynolds numbers and lift characteristics of finite wings of several aspect ratios with this airfoil at the same Reynolds numbers. The two-dimensional $C_{l^{-}} \alpha$ curves from the experimental data is used as input to the current method and the predicted finite-wing characteristics from both the schemes are compared with the corresponding experimental results. In comparison to the results from scheme 1 , the results from scheme 2 are found to be more acceptable. It is found that the wing $C_{L^{-}} \alpha$ for the examples presented agrees well with the experimental results till 30 degrees. Beyond 30 degrees the comparison with the experimental values is fair. As mentioned by Naik and Ostowari, ${ }^{17}$ in the experiments conducted to generate the airfoil data, the two-dimensional blade model was attached only at one end due to which it experienced some bending and twisting effects at high Reynolds numbers and high $C_{l}$ conditions. However, no attempt was made in Ref. 17 to correct the data for these effects. Because this two-dimensional data is used as input to the current method, any errors in the input data will result in errors in the predicted wing characteristics.

Comparison of the results from scheme 2 with scheme 1 shows that scheme 2 eliminates oscillations in the spanwise section $C_{l}$ distributions that were found in the results of Scheme 1 and were caused by the occurrence of regions of unstalled 
flow being sandwiched between regions of stalled flow. As with other methods, multiple solutions are possible for wings at high angles of attack and the solution is sensitive to the choice of the initial conditions for the iteration process. However, for certain angles of attack, sawtooth oscillations in the spanwise $C_{l}$ distributions from scheme 2 are observed in the stalled regions of the wing. It is shown that this sawtooth oscillation, also reported by other investigators, occurs because of alternate sections in the stalled region having converged to the unstalled portion of the airfoil $C_{l^{-}} \alpha$ curve. Although such solutions are numerically converged, they are not physically realizable. Thus, scheme 2, like many of the earlier ones, is not guaranteed to converge to physically-realizable solutions for every $\alpha$ of every geometry and airfoil combination.

In the second set of examples, the current method with scheme 2 is used to study the stall characteristics of wings with different taper ratios. The method predicts that rectangular wings stall first at the root, whereas tapered wings start stalling at the outboard portions. These trends correlate well with well-known effects of taper on the stall behavior. An example of a part-tapered planform is used to illustrate the stall behavior for such a planform. The third set of examples presents the post-stall characteristics for a wing-tail configuration. These examples shows how the current method is capable of predicting the effects of wing stall on the aircraft pitching moment behavior. The fourth set of examples presents the results for a wing-canard configuration to illustrate the stall behavior and the effect of the canard lift distribution on the wing aerodynamics.

Researchers in the past have reported asymmetry in the lift distributions even when the starting conditions were perfectly symmetric. The fifth example in this study was used to examine if such asymmetric behavior was seen with the current method. The results with scheme 2 did not exhibit any such asymmetries even when the starting conditions were asymmetric. 
The approach developed in this research uses a novel scheme (scheme 2) for computing the residuals for the iterative prediction of wing post-stall characteristics using section data. The examples show that this scheme 2 is more robust than scheme 1, which is similar to the schemes used by other researchers. An important new feature of scheme 2 is the determination of multiple target $C_{l}$ values during the iterative process for sections that are close to stall. The algorithm developed for choosing the desired target $C_{l}$ from among the multiple solutions enables solution of the equations for post-stall angles of attack. Good comparison with experimental data for pre-stall conditions and fair comparison with the experimental data for post-stall conditions indicates that the method can be used with reasonable confidence for the difficult task of rapid post-stall prediction of wing characteristics. Such rapid prediction method can be valuable in the design of new multiple-lifting-surface configurations. While significant progress has been made with the development of the new scheme in this research, some convergence problems still remain for certain airfoil lift curves and some angles of attack. Such problems have also been reported with other methods. ${ }^{7}$ Further research is needed to sort such problems before rapid post-stall predictions can be used routinely in aircraft design. 


\section{Chapter 6}

\section{Future Work}

This research was undertaken with the objective of developing a numerical prediction method that can rapidly provide post-stall data for multiple-lifting-surface configurations, where each lifting surface may have different planform shapes and different camber. In doing so, the only experimental data for both airfoil and finite wings for post-stall conditions found for comparison and validation was that of Naik and Ostowari. ${ }^{17}$ Even then, only the wing $C_{L}$ could be compared since no experimental data for the spanwise section $C_{l}$ distributions were found. Therefore, this work calls for experimental pressure measurements along the surface of a wing so that the experimental spanwise section- $C_{l}$ distributions can be calculated.

An important extension of this work will be to perform the unsteady analysis of a lifting surface. For this the wake behind the wing will also have to be discretized. The strength of the trailing vortices will be time-dependent and also depend on the vorticity shed by the wing.

Future work will involve improving upon the modeling technique used to get a measure of the effective decambering due to separated flow in the present VLM3D. This can be done by defining the separated flow using a cubic camberline instead of two linear functions.

In this work, the two-valued decambering function is evaluated using a Newtons's method. The Newton's method is a standard root polishing algorithm. 
The convergence of such an algorithm depends on the starting guess for the location of the root. Such a method is fast but less robust. When the behaviour of the function is compatible with the algorithm and a good initial guess is available, the Newton's method can provide rapid convergence. The Newton's method worked well for the airfoil data used in this work and convergence in all cases was obtained within a tolerance of 0.001 . However, using scheme 2, possibilities of multiple solutions arise and the Newton's method fails to converge for a few cases, and follows an undamped periodic motion. In other words, the Newton's method has problems finding the appropriate root in the multiple solution zone in some cases. This can be avoided by using a root bracketing method like the bisection method or the regula falsi method. Such methods are guaranteed to converge since these algorithms begin with a bounded region known to contain a root. The size of the bounded region is reduced, iteratively, until it finds a root within the allowable tolerance. This provides rigorous error estimation for the location of the root.

Therefore, the Newton's method can be replaced by the bisection method and the behaviour of the solution in the multiple solution zone can be studied. The bisection method will look for a root $\left(\alpha_{s e c}\right)$ within the minimum and maximum $\alpha$ of the $C_{l^{-}} \alpha$ curve provided as input.

The bisection method is less efficient than the Newton's method but it is guaranteed to converge and less prone to odd behaviour. It is thus believed that using the bisection method, the problem of a few angles of attack not converging as explained in detail in sec. 4.1 can be removed. 


\section{Appendix A}

\section{VLM3D}

The code VLM3D is developed to implement the methodology explained in Chapter 3. The following sections describe in detail the various components of VLM3D. In order to calculate the velocity induced at an arbitrary point $P(x p, y p, z p)$ due to a ring vortex, two subroutines "vortexring" and "horse-shoe-vortex" are developed. Subroutine "vortexring" calculates the induced velocity by adding the contributions of the four sides of the vortex ring, each of which is a finite vortex segment. Subroutine "horse-shoe-vortex" calculates the induced velocity of a horse-shoe vortex by adding the contributions of a finite vortex segment and two semi-infinite trailing vortices. When the ring vortex is at the trailing edge, both subroutines are used to calculate the induced velocity. The horse-shoe vortex is formed by the trailing segment of the vortex ring and two trailing vortices at its two ends.

\section{A.0.1 Subroutine readgeom}

This subroutine reads in the geometry parameters of a wing and/or its configurations. The airfoil geometry can be generated or can also be provided as an input. Both symmetric and cambered airfoils can be used. 


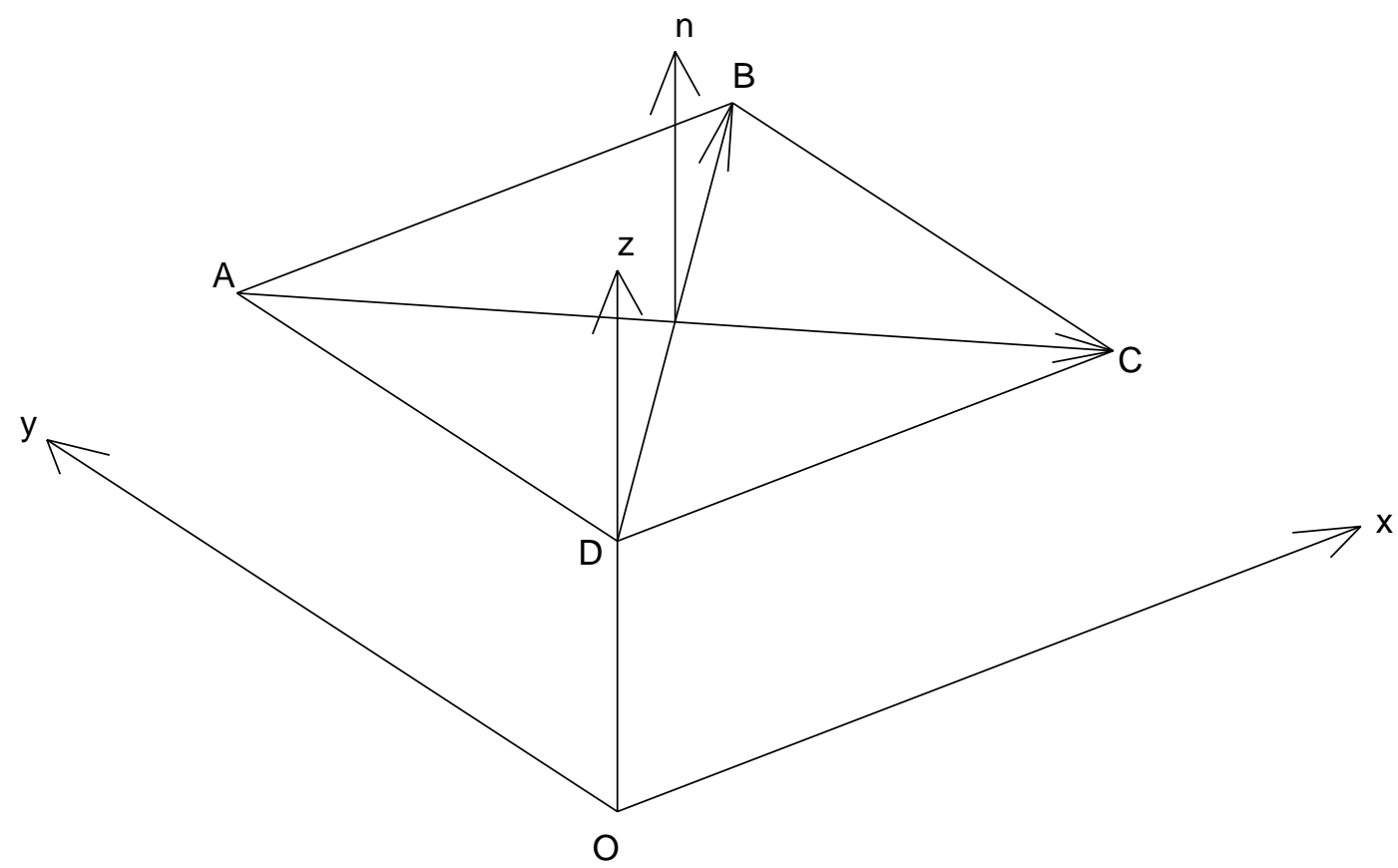

Figure A.1: Unit normal to a lattice.

\section{A.0.2 Subroutine geomgen}

This subroutine generates the geometry of the wing and/or its configurations for a given airfoil geometry. Rectangular and tapered wings can be generated. It divides the lifting surfaces into quadrilateral lattices for a given number of sections along span and along chord. For each lattice, its four vertices, the control point, vertices of the vortex ring and the unit normals are generated. The unit normals are generated by calling the subroutine panel-unitnormal. If a wing(s) has an incidence, geometric twist or aerodynamic twist, the effect is simulated by rotating the normals. The normals are rotated by calling the subroutine rotate-normalsingle-flap. A brief description of panel-unitnormal and rotate-normal-single-flap follow:

panel-unitnormal: This subroutine generates the unit normal to the panel by taking the vector cross product of the two vectors $\overrightarrow{A C}$ and $\overrightarrow{D B}$ as shown in Fig. A.1. 
rotate-normal-single-flap: This subroutine rotates the unit normal to a lattice. It is to be remembered that for all cases considered in this research work the component of the unit normal in the $y$-axis does not get affected. Therefore, only the components of the unit normal in the $x$ and $z$ axes are rotated. The following relations are used to rotate the normals:

$$
\begin{gathered}
U_{n x}{ }^{\text {new }}=U_{n x}{ }^{\text {old }} \cos \theta+U_{n z}{ }^{\text {old }} \sin \theta \\
U_{n z}{ }^{\text {new }}=-U_{n x}{ }^{\text {old }} \sin \theta+U_{n z}{ }^{\text {old }} \cos \theta
\end{gathered}
$$

where $\theta$ is the angle of rotation, $U_{n}$ denotes the unit normal and $x$ and $z$ denote the axis.

\section{A.0.3 Subroutine influence}

This subroutine calculates the influence coefficients of the wing and/or its configuration under consideration. Essentially, the influence coefficient at a particular point is the total induced velocity due to all vortices in its vicinity. In this case, the total induced velocity due to all the ring vortices and the horse-shoe vortices at the trailing edge at the control point of a lattice is the influence coefficient of that particular lattice. The influence coeficients depend only on the geometry. To calculate the induced velocity due to a ring vortex, the subroutine vortexring is called and to calculate the induced velocity due to a horse-shoe vortex at the trailing edge the subroutine horse-shoe-vortex is called. A brief description of vortexring and horse-shoe-vortex follow:

vortexring: This subroutine calculates the velocity induced due to a vortex

ring. It calls the subroutine vortex four times for the four segements of the vortex ring and sums up the contribution of all four segments to get the net contribution 


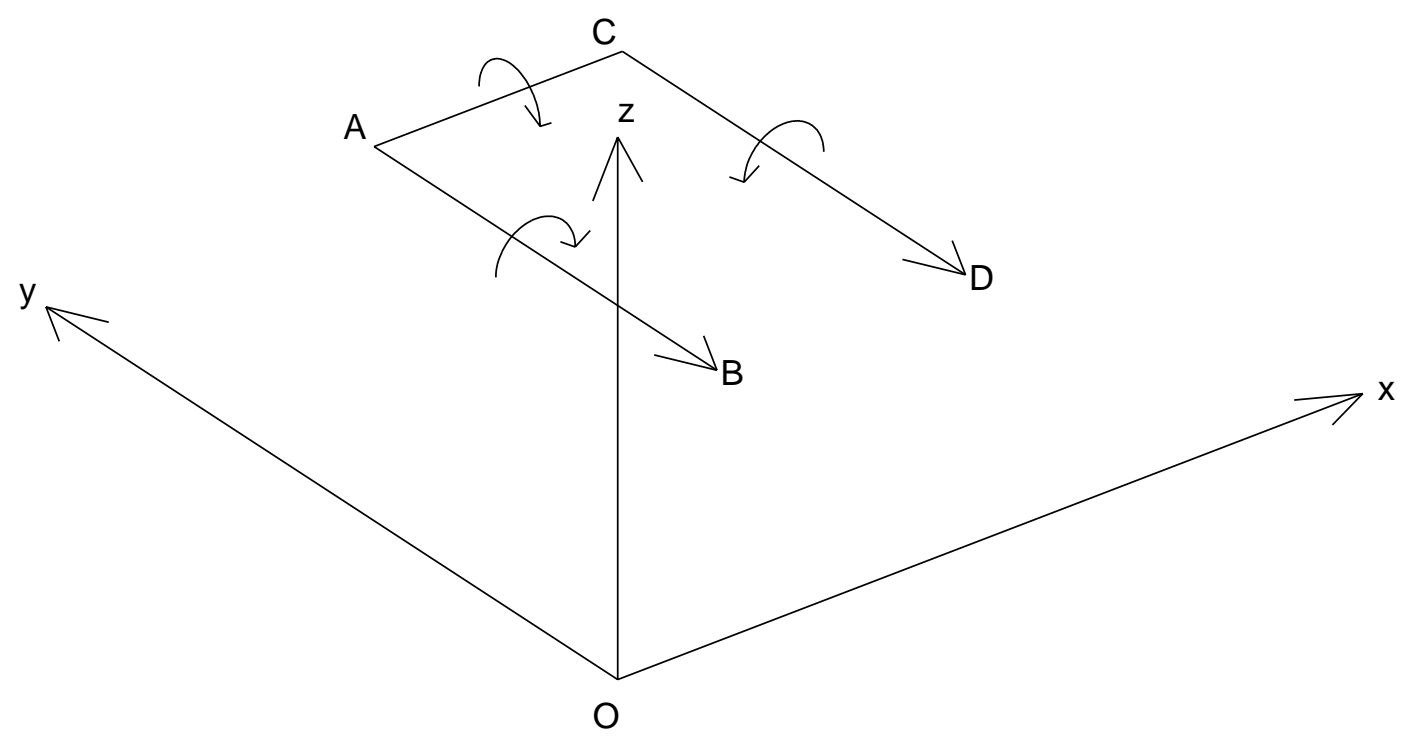

Figure A.2: Horse-shoe vortex at the trailing edge.

of the vortex ring. The subroutine vortex calculates the velocity induced by a finite vortex segment at a point $[x p, y p, z p]$ using the following relation:

$$
\overrightarrow{V_{\text {ind }}}=\frac{\left(\left|\overrightarrow{r_{1}}\right|+\left|\overrightarrow{r_{2}}\right|\right)\left(1-\frac{\overrightarrow{r_{1}} \overrightarrow{r_{2}}}{\left|\overrightarrow{r_{1}}\right|\left|\overrightarrow{r_{2}}\right|}\right)}{4 \pi\left|\overrightarrow{r_{1}} X \overrightarrow{r_{2}}\right|^{2}}\left(\overrightarrow{r_{1}} X \overrightarrow{r_{2}}\right)
$$

horse-shoe-vortex: This subroutine calcultes the induced velocity due to a horse-shoe vortex. It calls the subroutines vortex and trailing-vortex to calculate the net velocity due to a horse-shoe vortex. The subroutine trailing-vortex calculates the induced velocity due to the two trailing vortices of a horse-shoe vortex using the following relation:

$$
\overrightarrow{V_{i n d}}=\frac{1-\frac{\overrightarrow{r_{1}} \overrightarrow{U_{n}}}{\left|\overrightarrow{r_{1}}\right|}}{4 \pi\left|\overrightarrow{r_{1}} X \overrightarrow{U_{n}}\right|^{2}}\left(\overrightarrow{r_{1}} X \overrightarrow{U_{n}}\right)+\frac{1-\frac{\overrightarrow{r_{2}} \overrightarrow{U_{n}}}{\left|\overrightarrow{r_{2}}\right|}}{4 \pi\left|\overrightarrow{r_{2}} X \overrightarrow{U_{n}}\right|^{2}}\left(\overrightarrow{r_{2}} X \overrightarrow{U_{n}}\right)
$$

where the first part of the right hand side is the induced velocity due to the semi-infinite vortex $A B$ at the point [xp,yp,zp] and the second part of the right hand side is the induced velocity due to the semi-infinite vortex $C D$ at the point [xp,yp,zp] as shown in Fig. A.2. 


\section{A.0.4 Subroutine readoper}

This subroutine reads in the operating conditions of the wing and/or its configurations. Some of its salient features can be briefly described as follows:

Based on the inputs as per readoper, VLM3D can operate in the linear or non-linear mode and at a single $\alpha$ or at an $\alpha$-sequence. When operating at an $\alpha$-sequence, it has an added option to calculate the influence coefficients only once for the entire operation. When operating in non-linear mode, it can implement scheme 1 or 2 and initialise $\delta_{1}$ and $\delta_{2}$ at the first $\alpha$ of an $\alpha$-sequence or at each $\alpha$ of an $\alpha$-sequence.

\section{A.0.5 Subroutine dorhs}

This subroutine calculates the right hand side of the eqn. 3.1 based on the operating angle of attack and the free-stream vector.

\section{A.0.6 Subroutine iteration}

The entire non-linear analysis is executed by this subroutine. It calculates the jacobian matrix explained in section 3.2 and executes scheme 1 or scheme 2 as per requirement. To calculate the experimental $C_{l}$ and $C_{m}$ corresponding to the local section angle of attack, $\alpha_{e f f}$, subroutine data is called for scheme 1 and subroutine locate-alfa-intersect is called for scheme 2. Subroutine rotate-normals is used to rotate the normals of each lattice to account for the flap deflections, $\delta_{1}$ and $\delta_{2}$. Using scheme 2, this subroutine identifies unstalled sections with multiple solutions sandwiched between stalled sections and resets them as stalled sections. This removes the oscillations in the spanwise section $C_{l}$ distributions considerably. It solves the matrix eqn. 3.2 to yield new values of the flap deflections. For each angle of attack this subroutine performs the entire iteration process described in 
section 3.2. A brief description of subroutines data and locate-alfa-intersect follow: data: For a given $\delta_{1}$ and $\delta_{2}$ and the potential $C_{l}$ and $C_{m}$, the local section angle of attack, $\alpha_{e f f}$ is calculated using eqn. 3.7 in this subroutine. Corresponding to this angle of attack the experimental values of $C_{l}$ and $C_{m}$ are calculated. These are the target values for scheme 1 .

locate-alfa-intersect: In this subroutine, for a given $\delta_{1}$ and $\delta_{2}$ and the slope of the trajectory line, the local section angle of attack, $\alpha_{\text {eff }}$ is calculated by scanning the entire input airfoil $C_{l}$ data. Corresponding to this angle of attack the experimental values of $C_{l}$ and $C_{m}$ are calculated. These are the target values for scheme 2 . 


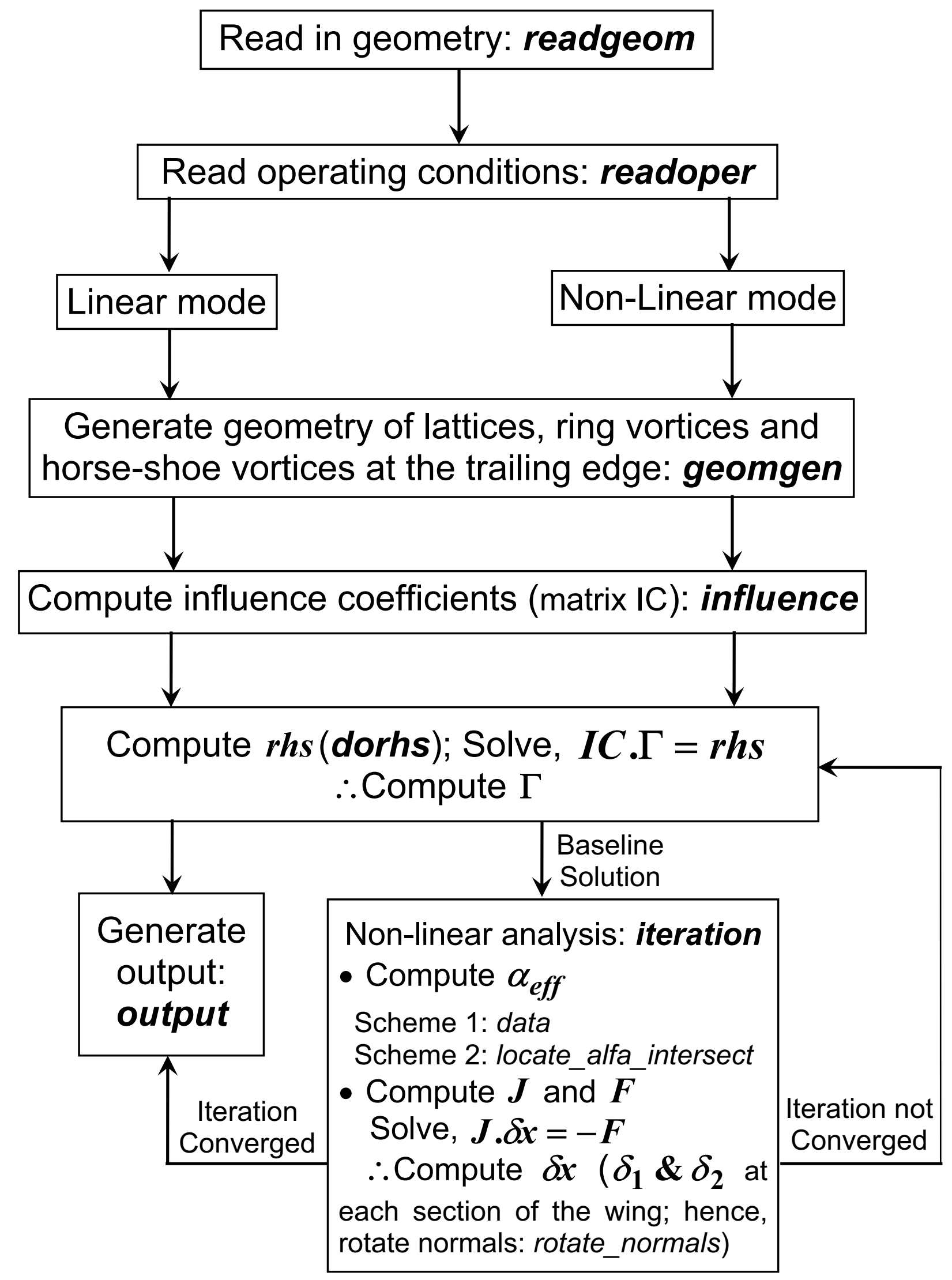

Figure A.3: Flow chart of VLM3D. 


\section{References}

1 Tani, I., "A Simple Method of Calculating the Induced Velocity of a Monoplane Wing," Rep. No. 111(vol. 9, 3), Aero. Res. Inst., Tokyo Imperial Univ., August 1934.

2 Sivells, J. C. and Neely, R. H., "Method for Calculating Wing Characteristics by Lifting-Line Theory Using Nonlinear Section Lift Data," NACA TN 1269, April 1947.

3 Schairer, R. S., Unsymmetrical Lift Distributions on a Stalled Monoplane Wing, Master's thesis, California Institute of Technology, 1939.

4 Sears, W. R., "Some Recent Developments in Airfoil Theory," Journal of The Aeronautical Sciences, Vol. 23, May 1956, pp. 490-499.

5 Piszkin, S. T. and Levinsky, E. S., "Nonlinear Lifting Line Theory for Predicting Stalling Instabilities on Wings of Moderate Aspect Ratio," Tech. rep., General Dynamics Convair Report CASD-NSC-76-001, June 1976.

6 Levinsky, E. S., "Theory of Wing Span Loading Instabilities Near Stall," AGARD Conference Proceedings No. 204, September 1976.

7 Anderson, M. R., "Aerodynamic Modeling for Global Stability Analysis," AIAA Paper 2002-4805, August 2002. 
8 Anderson, J. D., Corda, S., and VanWie, D. M., "Numerical Lifting Line Theory Applied to Drooped Leading-Edge Wings Below and Above Stall," Journal of Aircraft, Vol. 17, No. 12, 1980, pp. 898-904.

9 McCormick, B. W., "An Iterative Non-Linear Lifting Line Model for Wings with Unsymmetrical Stall," SAE Transactions Paper No. 891020, 1989, pp. 9198.

10 Tseng, J. B. and Lan, C. E., "Calculation of Aerodynamic Characteristics of Airplane Configurations at High Angles of Attack," NASA CR 4182, 1988.

11 van Dam, C. P., Kam, J. C. V., and Paris, J. K., "Design-Oriented High-Lift Methodology for General Aviation and Civil Transport Aircraft," Journal of Aircraft, Vol. 38, No. 6, November-December 2001, pp. 1076-1084.

12 Mukherjee, R., Gopalarathnam, A., and Kim, S., "An Iterative Decambering Approach for Post-Stall Prediction of Wing Characteristics Using Known Section Data," AIAA Paper 2003-1097, January 2003.

13 Mukherjee, R. and Gopalarathnam, A., "Post-Stall Prediction of MultipleLifting-Surface Configurations Using a Decambering Approach," AIAA Paper 2004-0219, January 2004.

14 Katz, J. and Plotkin, A., Low-Speed Aerodynamics, Cambridge Aerospace Series, Cambridge University Press, Cambridge, UK, 2001.

15 Drela, M., "XFOIL: An Analysis and Design System for Low Reynolds Number Airfoils," Low Reynolds Number Aerodynamics, edited by T. J. Mueller, Vol. 54 of Lecture Notes in Engineering, Springer-Verlag, New York, June 1989, pp. 1-12. 
16 Press, W. H., Teukolsky, S. A., Vetterling, W. T., and Flannery, B. P., Numerical Recipes in Fortran — The Art of Scientific Computing, Cambridge University Press, New York, 2nd ed., 1992, pp. 372-375.

17 Ostowari, C. and Naik, D., "Post Stall Studies of Untwisted Varying Aspect Ratio Blades with an NACA 4415 Airfoil Section-Part 1," Wind Engineering, Vol. 8, No. 3, 1984, pp. 176-194. 